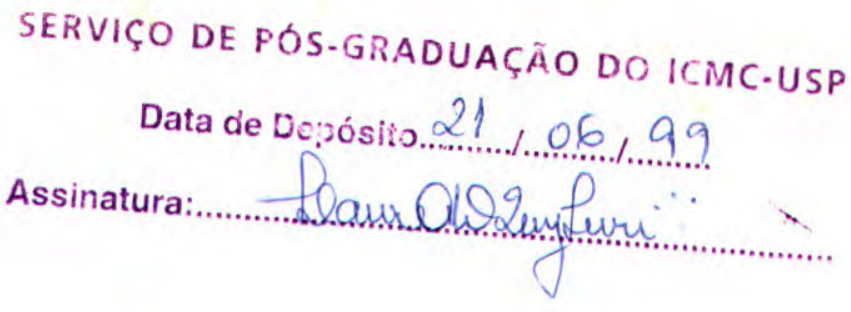

\title{
META-ANÁLISE: UM ENFOQUE BAYESIANO
}

\author{
Viviane Carla Fortulan \\ Orientação: Prof. Dr. Jorge Alberto Achcar
}

Dissertação apresentada ao Instituto de Ciências Matemáticas e de Computação - USP, como parte dos requisitos para a obtenção do titulo de Mestre em Ciências - Area de Ciências de Computação e Matemática Computacional 
Fo meu pai (onde quer que ele esteja). ¿̀ minbo mäezinba, D. Marli. à minbo imnä, Cristiane e co meu noioo Poulo. 


\section{Agradecimentos}

\section{Agradeço...}

Claro, em primeiro lugar, à Deus, que está presente sob oárias formas, em todas as etapas da minbo vida.

Ao meu maior incentioador, onde quer que o senbor esteje, sua presença ainda continua muito forie na minba vida. Obrigada por tudo, pai.

Ao Professor Dr. Dorge Alberio $\mathscr{A}_{c}$ cbcar pela atenção, pela orientação segura e por todo incentioo durante este periodo de mestrado.

Ao Professor Dr. Zosemar Rodrigues, que além das oaliosas sugestōes apresentadas em meu Exame de Qualificação, me orientou como monitora de duas disciplinos oferecidas à graduação. dando um exemplo de profissionalismo.

Ao Professor Dr. Dorge Otsbi, que acompanbou minba jomada desde a graduação sempre motioando aos estudas e pelas excelentes sugestōes apresentadas em meu Exame de Qualificação.

Ao Professor Dr. Marinbo Gomes de Andrade Filbo pela amizade, pelo pronto atendimento e auxíio nos programos computactonais.

Ao Professor Poti e a sua esposa Goani por toda ajuda que me prestaram.

$\grave{A}$ todos as meus professores e amigos da Universidade Federal de São Carlos, pelo incentioo, dedicação e, principalmente, pela grande amizade que continua até boje.

$\mathscr{A}_{0}$ CSPP q è APPESP, pelo apoio financeiro. 
Ros funcionários do $\mathscr{C}$ MC principalmente às meninas da Secretaria de Pás-Graduação. Bbety. Laura e Marilia.

भo meu grande amigo Ouon Esteban Ramirez Cid pela omizade. e pelos diversos ensinamentos.

Aos meus amigos do curso, em especial, oo Jasenildo S. Cbaves, à Sandra C. de Oliveira.

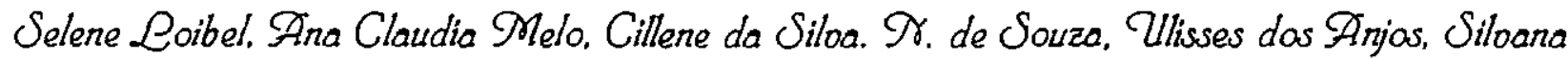
Meira. Gilberto Pereira. Gilberto Rinaldi e Daniela Brassolatti.

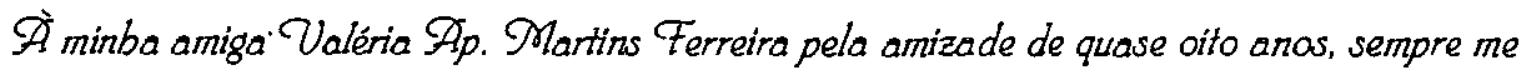
incentioando e ajudando em tudo que é passioel.

$\mathscr{A}_{0}$ Paulo César Tricola, pelo amor, compreensāo, paciência e incentioo em todos as momentos.

$\mathscr{A}$ tode minba família. em especial, a minbe mëe. Marli. minba irmä. Critiane, aos meus primos, Carlos Riberto Fortulan e Marcos Roberto Fortulan, a minba tia Mirian C. Lopes Franco e meu tio Sergio Franco. Obrigada pelo incentioo, atenção e por todo carinbo que recebi. 


\section{RESUMO}

Apresentamos neste trabalho o uso de meta-análise sob um enfoque Bayesiano. Metaanálise se refere a métodos quantitativos que combinam resultados de estudos independentes para tirar conclusões gerais. Freqüentemente resultados de diferentes estudos são combinados de forma inadequada, resultando em uma análise inferencial não confiável. Métodos Bayesianos para meta-análise são preferiveis em vista do pequeno número de estudos que prevalecem em meta-análise. Para exemplificar o uso desta técnica, utilizamos alguns conjuntos de dados disponíveis na literatura e um conjunto de dados simulado. A análise Bayesiana foi possível devido a utilização de métodos de simulação de Monte Carlo via Cadeias de Markov. 


\begin{abstract}
This work is concerned with the use of meta-analysis from a Bayesian point of view. Meta-analysis is a quantitative method combining independent results to obtain global conclusions. Frequently different results are combined unapropriately leading to an unreliable inferential analysis. Bayesian methods for meta-analysis are preferred due to the small number of experiments which are caracteristc of this technique. Sets of data taken from the literature, were used to illustrate the use of the technique, and a simulation set. A Bayesian analysis is made possible through the use of Monte Carlo simulation methods via Marcov Chains.
\end{abstract}




\section{ÍNDICE}

Capítulo 1 - Introdução 1

1.1 Definição........................................................................................... 1

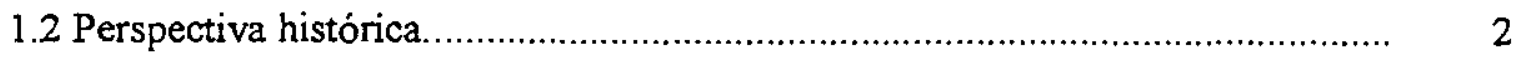

1.2.1 Combinação de testes de hipóteses................................................ 3

1.2.2 Estimą̧ão combinada............................................................... 5

1.3 Vantagens do uso do modelo Bayesiano em meta-análise................................. 6

1.3.1 Exemplos de aplicações em meta-análise sob um enfoque Bayesiano. 8

1.4 Objetivos principais da pesquisa................................................................. 9

Capítulo 2 - Uma introduçāo à inferência Bayesiana 10

2.1 Fórmula de Bayes.................................................................................... 10

2.2 Densidades a priori e a posteriori................................................................ 12

2.3 Algumas considerações sobre seleção de modelos........................................... 13

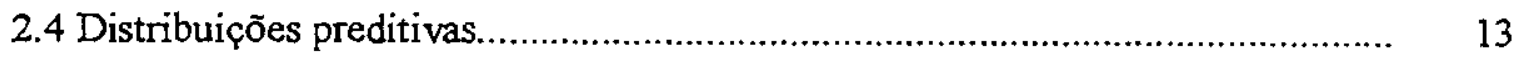

2.4.1 Densidade preditiva condicional ordenada (CPO) ......................... 14

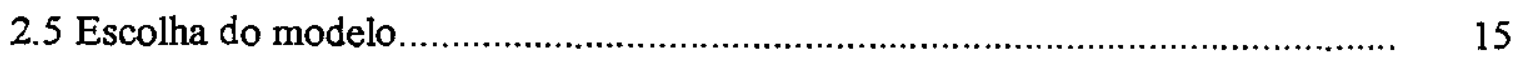

2.6 Verificação da adequação do modelo via densidade preditiva........................... 15

2.6.1 Procedimento para verificaçåo do modelo utilizando o nivel

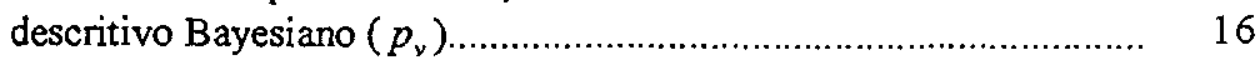

Capitulo 3 - Estudo sobre o uso de uma nova droga contra epilepsia 19

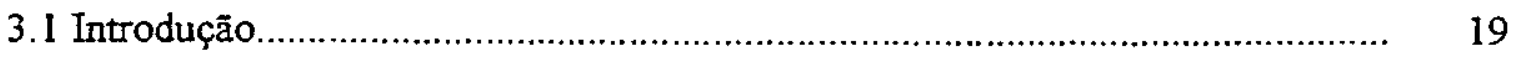

3.2 Definição do modelo e suas respectivas variáveis.............................................. 21

3.2.1 Função de verossimilhança............................................................. 23

3.2.2 Distribuição a priori................................................................. 24

3.2.3 Distribuição a posteriori conjunta................................................ 26

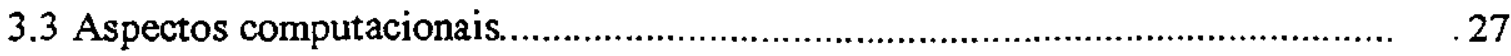




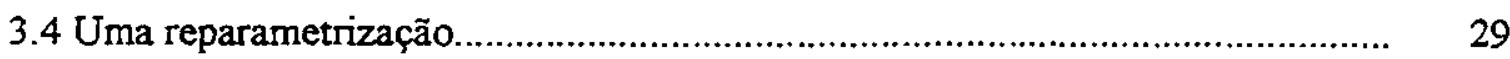

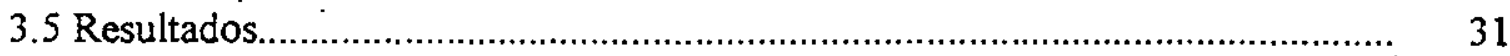

3.5.1 Análise gráfica.......................................................................... 34

3.6 Verificação da adequação do modelo................................................................. 40

Capítulo 4 - Um procedimento clássico em meta-análise $\quad 41$

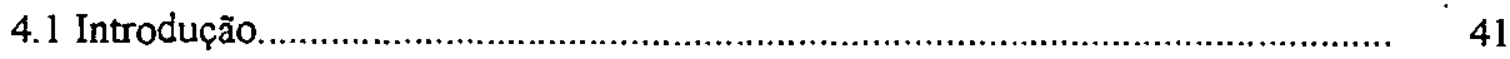

4.2 Comparą̧ão de proporções de várias amostras independentes............................. 41

4.2.1 Teste para verificar a igualdade de $N$ proporções.................................. 43

4.2.2 Tese para verificar a igualdade entre dois grupos de estudo................. 44

4.2.3 Tese para verificar a igualdade dentro de grupos................................ 45

4.2.4 Teste para verificar se cada uma das $N$ proporções amostrais é igual

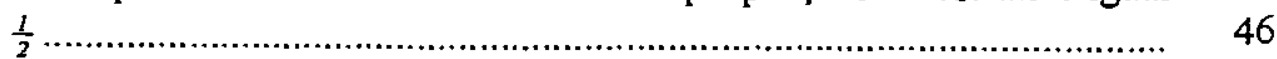

4.3 Intervalos com $95 \%$ de confiança para cada uma das $N$ proporções..................... 47

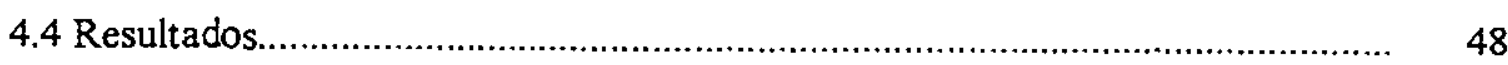

Capítulo 5 - Um exemplo simulado para ilustrar a comparação entre dois materiais 52 usados para fabricar solas de sapatos

5.1 Introdução

5.2 Definição do modelo 1 e suas respectivas variáveis........................................... 54

5.2.1 Função de verossimilhança................................................................ 55

5.2.2 Distribuição a priori........................................................................... 55

5.2.3 Distribuição a posteriori conjunta....................................................... $\quad 56$

5.2.4 Aspectos computacionais................................................................ 56

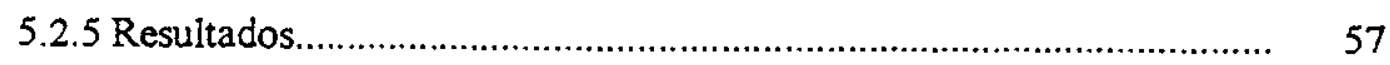

5.2.6 Análise gráfica.......................................................................... 58

5.3 Definição do modelo 2 e suas respectivas variáveis........................................... 61

5.3.1 Função de verossimilhança................................................................ 62

5.3.2 Distribuição a priori.......................................................................... 62

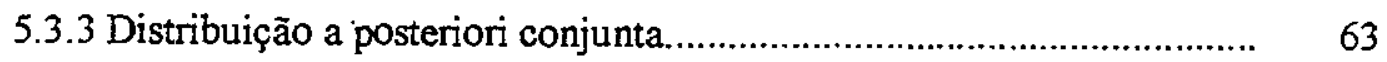

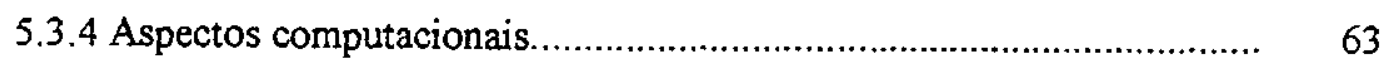

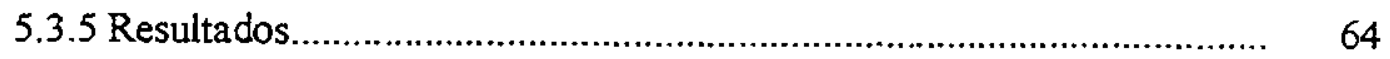

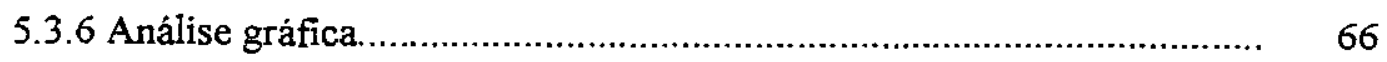

5.4 Escolha e verificação do ajuste do modelo....................................................... 71 


\section{META-ANÁLISE: UM ENFOQUE BAYESIANO}

\section{CAPITULO 1 - INTRODUÇÃO}

\subsection{Definição}

Podemos definir meta-análise como uma aproximação quantitativa de combinações sistemáticas de resultados de várias pesquisas com o objetivo de buscar conclusões sobre o corpo da pesquisa.

O objetivo geral da meta-análise é combinar resultados de estudos anteriores e independentes com a finalidade de obter conclusōes gerais sobre a pesquisa. Esta técnica é muito útil quando o número de estudos sobre um determinado tema é pequeno e, além disso, esses estudos envolvem amostras pequenas para produzir uma conclusão válida.

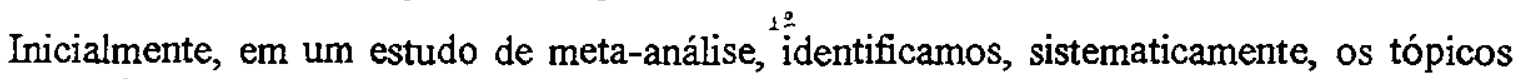

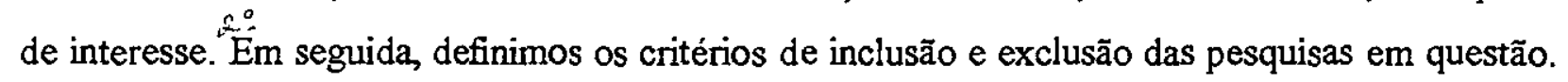
$3^{9}$ Por último, os dados, provenientes dos estudos selecionados, são resumidos e combinados, produzindo uma estimativa quantitativa do magnitude do efeito do tratamento.

O desafio dos estatísticos é, portanto, desenvolver metodologias eficientes para combinar informações de estudos relacionados e independentes para tirar conclusões gerais, isto é, desenvolver modelos estatísticos para meta-análise. 


\subsection{Perspectiva histórica}

O termo "meta-análise" foi criado por Glass em 1976 do prefixo grego "meta" que significa "transcender", e a raiz, análise. Glass procurou distinguir meta-análise a partir de uma análise primária - a análise original dos dados de uma pesquisa - e a análise secundária - a reanálise dos dados no sentido de responder novas questôes sobre a pesquisa (Glass 1976; Glass, McGraw, Smith 1981). No entanto, a combinação estatística de dados de estudos múltiplos de um mesmo tópico iniciou muito antes. Na década de 30, Tippett (1931), Fisher (1932), Cochran (1937) e Pearson (1938), descreveram técnicas estatísticas para combinar dados de diferentes estudos. Na literatura agrícola, por exemplo, o uso destas técnicas são numerosos.

A necessidade de técnicas para combinar dados de vários estudos de um mesmo tópico tornou-se especialmente forte nas ciências sociais na metade da década de 70 quando, literalmente, centenas de estudos eram feitos para determinados tópicos. Durante o final dos anos 70 e começo dos anos 80, cientistas sociais, incluindo Rosenthal (1978), Glass, McGraw e Smith (1981), Hedges (1982, 1983), Hunter, Schmidt e Jackson (1982), e Light e Pillemer (1984), popularizaram meta-análise e mais adiante desenvolveram métodos estatísticos para sua aplicação.

O primeiro uso da meta-análise na medicina é difícil de datar precisamente. No final dos anos 80 , descrições de métodos de meta análise apareceram quase simultaneamente em três jornais médicos influentes, o New England Journal of Medicine, Lancet, e o Annals of Internal Medicine (L'Abbe, Detsky, O’Rourke 1987; Sacks et al. 1987; Bulpitt 1988). O uso mais comum de meta-análise na medicina tem coincidido com o aumento de focos de pesquisa médica em ensaios clínicos aleatorizados, e tem, evidentemente, beneficiado o aumento no nível de interesse sobre a interpretação de pequenos ensaios clínicos e individualmente não conclusíveis.

Meta-análise tem sido aplicada mais freqüentemente na combinação de resultados de processos aleatorizados. No entanto, o uso de meta-análise não está limitado a síntese de informações de estudos experimentais. O uso de meta-análise também é comum em estudos não experimentais, isto é, em estudos nos quais processos aleatorizados não podem ser aplicados. Por exemplo, o uso de fumó e álcool não podem ser alocados aos individuos do estudo. Para estudos não experimentais, a meta-análise é mais útil quando existem vários estudos com baixo poder estatístico. 
Uma revisão da história da meta-análise também é dada por Hedges (1992). Neste aspecto pode-se considerar duas metodologias distintas: a combinação de testes de hipóteses e, mais recentemente, a combinação dos estimadores obtidos com vários tamanhos amostrais.

\subsubsection{Combinação de testes de hipóteses}

A primeira metodologia, "omnibus tests", combina resultados de testes de hipóteses, geralmente utilizando alguma função dos valores-p (ou "p-values"). Sendo assim, esta metodologia não depende da distribuição estatística dos dados e essa natureza não paramétrica dos "omnibus tests" pode gerar conclusões errôneas.

Exemplos deste tipo de metodologia são o "método do p mínimo" dado por Tippett (1931) e o "método do Qui-quadrado inverso" dado por Fisher (1932).

Considere uma coleção de $k$ estudos independentes caracterizados pelos parâmetros $\theta_{1}, \ldots, \theta_{k}$, tal como médias, diferença de médias, ou correlações. Assumir além disso que o i-ésimo estudo produz um teste estatístico contínuo $T_{i}$ usado para testar a hipótese nula,

$$
H_{o i}: \theta_{i}=0, \quad i=l, \ldots, k \text {, }
$$

onde grandes valores do teste estatístico levam a rejeição da hipótese nula. As hipóteses $H_{o l}, \ldots, H_{o k}$ não precisam ter o mesmo significado, e, similarmente, as estatísticas $T_{1}, \ldots, T_{k}$ não precisam estar de forma relatada. A hipótese nula $H_{0}$ é aquela onde todos os $\theta_{t}$ são iguais a zero, ou seja,

$$
H_{0}: \theta_{1}=\theta_{2}=\ldots=\theta_{k}=0
$$

Note que a hipótese $H_{0}$ é verdadeira somente se cada uma das subhipóteses $H_{01}, \ldots, H_{0 k}$ é verdadeira 
O valor-p para o i-ésimo estudo é

$$
p_{i}=\operatorname{Prob}\left\{T_{i}>t_{i o} \mid \theta_{i}=0\right\}
$$

onde $t_{i 0}$ é o valor da estatística obtida no i-ésimo estudo. Sob $H_{0 t}, p_{i}$ segue uma distribuição Uniforme $[0,1]$.

O método introduzido por Tippett é definido como segue: sejam $p_{i}, \ldots, p_{k}, k$ valores-p independentes (de testes estatísticos contínuos), onde, cada um deles tem distribuição Uniforme sob $H_{0}$. Desta forma, se $p_{[1]}$ é o mínimo de $p_{1}, \ldots, p_{k}$, um teste para $H_{0}$ com nível de significância $\alpha$ é obtido comparando $p_{[i]} \operatorname{com} 1-(1-\alpha)^{1 / k}$. Assim, rejeitamos a hipótese $H_{0}$ se $p_{[1]}<1-(1-\alpha)^{1 / x}$.

O método "método do Qui-quadrado inverso", é definido da seguinte forma: dado $k$ estudos independentes e $k$ valores- $\mathrm{p}, p_{1}, \ldots, p_{k}$, onde, sob $H_{o},-2 \log \left(p_{i}\right), i=1,2, \ldots k$, tem uma distribuição $\chi^{2}$ com 2 graus de liberdade, e, portanto, $-2 \log \left(p_{1} p_{2} \ldots p_{k}\right)=-2 \sum_{i=1}^{t} \log \left(p_{i}\right)$ tem uma distribuição $\chi^{2}$ com $2 k$ graus de liberdade. Assim, rejeitamos a hipótese $H_{0}$ se $-2 \sum_{i=1}^{k} \log \left(p_{i}\right)>c$, onde $c$ é obtido da cauda superior de uma distribuição $\chi^{2} \operatorname{com} 2 k$ graus de liberdade.

Existem algums inconvenientes para combinar testes de hipóteses. Um desses inconvenientes é que os "omnibus tests" não fornecem informações sobre o tamanho dos efeitos dos tratamentos, além de existir problemas com a interpretação dos resultados. Por exemplo, a hipótese nula geral $H_{0}: \theta_{1}=\theta_{2}=\ldots=\theta_{N}=0$ afirma que os efeitos dos tratamentos, em todos os estudos, são iguais a zero. Se $H_{0}$ é rejeitada no procedimento de testes combinados, seguramente, concluimos que $H_{0}$ é falsa. Portanto, $H_{0}$ é falsa se no mínimo um dos $\theta_{i}{ }^{\prime} s$, $i=1, \ldots, N$, é diferente de zero. Assim, $H_{0}$ é falsa quando, por exemplo, $\theta_{i}>0$ e $\theta_{2}=\ldots=\theta_{N}=0$. 
Os erros de interpretação são, geralmente, devidos a tentativa de dar uma interpretação paramétrica à rejeição de $H_{0}$. Por exemplo, um pesquisador pode incorretamente concluir que, uma vez que $H_{o}$ é rejeitado, os efeitos do tratamento são maiores que zero ou que a média dos efeitos do tratamento $\bar{\theta}$ é positiva.

Alguns exemplos deste tipo de metodologia e suas limitaçōes são dados com mais detalhes por Hedges (1992).

\subsubsection{Estimação combinada}

Quando todos os estudos têm padrão similar e os resultados são obtidos de maneira similar (não necessariamente idêntica), a estimação combinada é o método preferido da metaanálise (Hedges \& Olkin, 1985).

Essa segunda metodologia, a combinação de estimadores obtidos com vários tamanhos amostrais, minimiza as conclusões erradas que podem ser tomadas utilizando a combinação de testes de hipóteses mencionadas na Seção 1.2.1, porém, traz um inconveniente, pois os procedimentos utilizados nessa segunda metodologia são baseados na teoria de grandes amostras.

Assim como em análise de variância os modelos estatísticos podem ser definidos como modelos de efeitos fixos, aleatórios e mistos, em meta-análise temos, também, conceitos análogos. Os procedimentos para analisar dados nestes modelos são similares, mas a interpretação de testes estatísticos é um tanto diferente (ver Hedges \& Olkin, 1985).

"No conceito de efeitos fixos, os valores populacionais dos efeitos dos tratamentos são constantes fixas mas desconhecidas. Neste tipo de modelagem para meta-análise, os diferentes estudos são supostos homogêneos, medindo aproximadamente a mesma quantidade, sem variação entre estudos. Isto é, á resposta $y_{i}$ é modelada como $y_{i}=\mu+\varepsilon_{i}, \varepsilon_{i} \sim N\left(0, S_{t}^{2}\right)$, onde $S_{i}^{2}$ é a variância da resposta. Observe que a única fonte de variabilidade assumida é a variação dentro do estudo.

No caso da modelagem de efeitos aleatórios, os efeitos dos tratamentos variam aleatoriamente de estudo para estudo, como se fossem amostras de um universo de possíveis efeitos de tratamentos. Assim, é possível avaliar a variabilidade entre estudos a partir de componentes de variância. 
$\mathrm{Na}$ realidade, modelos de efeitos fixos estão presentes na maioria das modelagens em meta-análise. Porem, a rigorosa suposição de homogeneidade geralmente não é satisfeita na prática, como por exemplo, a existência de variabilidade entre centros médicos ou protocolos médicos é um fato. Além disso, estimativas super otimistas da variabilidade no modelo de efeitos fixos podem levar a conclusões erradas. Estes problemas podem ser evitados assumindo um efeito aleatório $\delta_{1}$, o qual explica a variabilidade entre estudos.

Assim, $y_{i}=\mu+\delta_{i}+\varepsilon_{i}$, onde $\delta_{i} \sim N\left(0, r^{2}\right)$ e $\varepsilon_{i} \sim N\left(0, S_{i}^{2}\right)$.

Note que se $\tau^{2}$. $=0$, então o modelo de efeitos aleatórios se reduz ao modelo de efeitos fixos (embora as diferenças na interpretação podem permanecer). No modelo de efeitos aleatórios, os efeitos observados não são assumidos homogêneos, mas representam amostras de uma população de efeitos. Porém, existem alguns problemas na formulação de efeitos aleatórios. O teste para determinar se $\tau^{2}=0$ não é extremamente confiável devido ao número pequeno de estudos que geralmente prevalecem em meta-análise (Fleiss, 1993).

A formulação de efeitos mistos seria uma combinação de efeitos fixos e efeitos aleatórios.

Estes métodos clássicos tais como os que envolvem a combinação das estimativas dos efeitos usam análise de máxima verossimilhança que precisam da disponibilidade de grandes amostras.

Daí surge a questão: "Como lidar com tamanhos amostrais pequenos prevalecendo em meta-análise?"

É importante salientar que trabalhamos com estatísticas que resumem os resultados de cada estudo, o que leva a tamanhos amostrais pequenos.

\subsection{Vantagens do uso do modelo Bayesiano em meta-análise}

O uso de técnicas Bayesiana oferece várias vantagens em modelagem deste tipo. A grande vantagem do uso de métodos Bayesianos está relacionada a uma grande precisão quando temos tamanhos amostrais pequenos, o que não ocorre com o uso de métodos assintóticos dos estimadores de máxima verossimilhança, ou seja, para grandes amostras, os resultados obtidos através dos métodos Bayesianos são próximos aos obtidos via máxima verossimilhança, e para tamanhos amostrais pequenos, os estimadores Bayesianos da variabilidade devem ser mais 
precisos do que os resultados obtidos via máxima verossimilhança. Além disso, oferecem uma estrutura conveniente para incorporar informações a priori disponiveis para melhorar a precisão da inferência.

Um outro fator muito importante é o grande número de parâmetros que geralmente estão presentes em uma pesquisa, o que torna o uso de métodos clássicos intratáveis.

A formulação Bayesiana oferece um mecanismo natural para descrever e explicar a heterogeneidade entre estudos individuais, assim como entre grupos de estudos, tais como estudos caso-controle e estudos abertos e fechados, enquanto que a meta-análise clássica envolve a combinação da magnitude dos efeitos.

Para ilustrar o uso da técnica Bayesiana considere a seguinte situação: Suponha que um estatístico esteja interessado em um efeito $\theta$, que para simplificação é suposto o mesmo em todos os estudos. Após a primeira análise, a distribuição a posteriori para $\theta$ é dada por,

$$
p\left(\theta \mid x_{2}\right)=\frac{p\left(x_{\sim} \mid \theta\right) p(\theta)}{\int_{\substack{f \\ \rho}}\left(x_{1} \mid \theta\right) p(\theta) d \theta}
$$

onde $x_{1}$ é o conjunto de dados do primeiro estudo, $p(\theta)$ é a distribuição a priori do efeito $\theta$, e $p\left(x_{\sim} \mid \theta\right)$ é a função de verossimilhança.

Para uma nova análise, podemos usar $p\left(\theta \mid x_{2}\right)$ como uma priori para uma próxima análise levando a,

$$
p\left(\theta \mid \underset{\sim}{x_{1}, x_{2}}\right) \propto p\left(x_{2} \mid \theta\right) p\left(\theta \mid x_{2}\right)
$$

e assim sucessivamente. 


\subsubsection{Exemplos de aplicações em meta-análise sob um enfoque Bayesiano}

Várias aplicações de meta-análise estão disponíveis na literatura, ver, por exemplo, Petitti (1994). Neste trabalho, vamos utilizar aplicações para ilustrar o uso da técnica sob um enfoque Bayesiano.

\section{Exemplo 1 - Estudo sobre o uso de uma nova droga contra epilepsia}

É comum um pesquisador estar interessado em comparar uma nova droga com uma tradicionalmente usada e observar se essa nova droga pode trazer sucesso na cura de uma determinada doença. O pesquisador pode estar interessado, por exemplo, na proporção de melhora que essa nova droga causa no estado clínico do paciente.

Neste contexto, será considerado um conjunto de dados introduzidos por Van Belle (1993) que traz 15 relatórios comparativos, independentes e agrupados de ensaios clínicos sobre uma nova droga antiepilética.

\section{Exemplo2 - Um exemplo simulado para ilustrar a comparação entre dois materiais usados para fabricar solas de sapatos}

Para ilustrar um caso comum de interesse industrial utilizamos um exemplo simulado onde uma indústria está interessada em saber se um novo material para fabricar solas de sapatos é superior a um tradicional.

O conjunto de dados utilizados para este exemplo foi gerado aleatoriamente utilizando o software MINITAB.

\section{Exemplo 3 - Relação entre o uso de hormônios e câncer em mulheres}

Nos últimos anos tem havido muita pesquisa médica para avaliar a possivel associação entre a incidência de câncer nos seios, útero e ovários e o uso de estrógeno (hormônios). Um conjunto de dados introduzido por Larose e Dey (1995 b) é considerado para verificarmos a existência de alguma relação entre estrógeno e câncer no útero, ovários e nos seios. 


\subsection{Objetivos principais da pesquisa}

O objetivo principal deste trabalho é desenvolver modelos Bayesianos para combinar informações de estudos comparativos e independentes. A partir de estudos deste tipo, várias questões de interesse podem ser respondidas utilizando a técnica estatística denominada metaanálise.

Como parte do objetivo, vamos explorar o uso dos algoritmos de simulação via cadeias de Markov conhecidos como algoritmo Gibbs Sampling e algoritmo Metropolis Hastings (ver por exemplo Casella \& George, 1992; Gamerman 1996; Apêndices 1 e 2).

No capitulo 2, apresentamos uma breve introdução à inferência Bayesiana incluindo algumas considerações sobre seleção de modelos e verificação do ajuste do modelo via densidade preditiva.

Através de alguns exemplos de aplicaçāo, considerando densidades a priori informativas para os parâmetros envolvidos nos modelos, desenvolvemos nos capítulos 3, 5 e 6, uma análise Bayesiana utilizando os algoritmos Gibbs Sampling e Metropolis Hastings para cada um dos modelos utilizados em cada um dos exemplos de aplicação introduzidos na Seção 1.3.

No capítulo 3, consideramos um modelo de efeitos aleatórios agrupados para meta-análise Bayesiana aplicado a um conjunto de dados introduzidos por Larose e Dey (1995 b) relacionado a eficácia de uma nova droga antiepilética. No capítulo 4 exemplificamos a aplicação de metaanálise clássica considerando a combinação de testes de hipóteses.

O capítulo 5 traz um exemplo simulado onde os dados foram gerados aleatoriamente utilizando o software MINITAB. Para estes dados consideramos dois modelos hierárquicos, um deles, considerando um possível efeito aleatório para os grupos.

No capítulo 6, um conjunto de dados introduzidos por Larose e Dey. (1995 b) é analisado através da meta-análise Bayesiana utilizando um modelo de regressão.

E finalmente, no capitulo 7, apresentamos as conclusões finais e perspectivas futuras deste trabalho. 


\section{CAPITTULO 2 - UMA INTRODUÇÃO À INFERÊNCIA BAYESIANA}

Métodos Bayesianos são métodos alternativos aos métodos clássicos de influência que podem melhorar a precisão e a interpretação dos resultados. Entre as diferenças importantes que existem entre os métodos clássicos e os Bayesianos salientamos: os métodos Bayesianos permitem a incorporação de informações a priori sobre os parâmetros do modelo, e, ao contrário dos métodos clássicos, os métodos Bayesianos consideram o parâmetro de interesse da distribuição uma variável aleatória, associando a ele uma distribuição de probabilidade.

Através da fórmula de Bayes, que combina a informação vinda dos dados através da verossimilhança com a incorporação de conhecimentos a priori, ou seja, com as informações obtidas antes dos dados, obtém-se uma distribuição denominada distribuição a posteriori. A partir desta distribuição a posteriori é possível determinar as quantidades necessárias para o processo inferencial, tais como medidas de posição e dispersão.

\subsection{Fórmula de Bayes}

Assim como na inferência frequentista, a inferência Bayesiana trabalha na presença de observações $y$ cujo valor é inicialmente incerto e descrito através de uma distribuição de probabilidade com densidade de probabilidade $p(y \mid \theta)$. Sob o ponto de vista Bayesiano, a quantidade $\theta$ é considerada uma variável aleatória, representando características de interesse que se deseja conhecer para poder ter uma descrição completa do processo.

Neste sentido, sendo y e $\theta$ consideradas variáveis aleatórias, ambas podem ser expressas através de uma distribuição de probabilidade. Assim como a distribuição de $y$, a distribuição de 
$\theta$ também pode ser especificada com o auxílio de constantes. Para distingui-las do parâmetro $\theta$ de interesse, estas constantes são chamadas de hiperparâmetros pois são parâmetros das distribuição dos parâmetros. Geralmente os hiperparâmetros são considerados conhecidos.

Desta forma, conhecendo a distribuição das observações $p(y \mid \theta)$ e a distribuição a priori de $\theta$, denotada por $p(\theta)$, é razoável que o processo de inferência seja baseado na distribuição de probabilidade de $\theta$ após observar o valor de $y$, essa distribuição é chamada de distribuição a posteriori em direta oposição à priori, podendo ser obtida através do Teorema de Bayes da seguinte forma,

$$
p(\theta \mid y)=\frac{p(y \mid \theta) p(\theta)}{p(y)}
$$

onde $p(y)=\int p(y \mid \theta) p(\theta) d \theta$ recebe o nome de distribuição preditiva (ou marginal) de $y$ pois é a distribuição que se espera que $y$ tenha sendo de certa forma uma predição.

Como $p(\theta \mid y)$ é uma densidade para $\theta$, a observação y é apenas uma constante bem como $p(y)$. Logo, o Teorema de Bayes pode ser escrito em uma forma mais resumida como,

$$
p(\theta \mid D) \propto L(\theta) p(\theta)
$$

onde $D$ denota o conjunto de observações y e $L(\theta)$ é chamada de função de verossimilhança de $\theta$. Essa função (de verossimilhança) associa (para um valor fixo de $y$ ) a probabilidade de ser observado y a cada valor de $\theta$.

Mudando um pouco a notação para um melhor discernimento das distribuiçōes mencionadas numa análise Bayesiana, denotaremos as distribuições a priori e a posteriori respectivamente por $\pi(\theta)$ e $\pi(\theta \mid y)$. Desta forma, a expressão (2.2) poderia ser rescrita como,

$$
\pi(\theta \mid D) \propto L(\theta) \pi(\theta)
$$




\subsection{Densidades a priori e a posteriori}

Em uma análise Bayesiana é possivel incorporar conhecimentos prévios a respeito da quantidade $\theta$ através de uma densidade $\pi(\theta)$. Essa densidade denotada por $\pi(\theta)$ recebe $o$ nome de densidade a priori.

Definido o modelo para os dados e a distribuição a priori, combinamos a informação contida nos dados com a informação prévia sobre o parâmetro $\theta$ e obtemos assim a distribuição a posteriori para $\theta$.

A partir da distribuição a posteriori de $\theta$, podemos determinar as quantidades de interesse necessárias para o processo inferencial tais como medidas de posição (média, moda e mediana) e dispersão (desvio-padrão, variância, etc.).

Uma das limitações dos métodos Bayesianos nas aplicações, em geral eram relacionadas à integração da densidade conjunta a posteriori para obtenção das distribuições marginais, que na maioria das vezes é muito complexa e só pode ser feita de forma aproximada. Nesses casos, o uso de métodos de aproximação como por exemplo, o método de Laplace para aproximações de integrais são necessários (ver por exemplo, Tierney, L. \& Kadane, J. B., 1986).

Neste sentido, uma alternativa muito usada recentemente, é a utilização de técnicas de simulação, como os métodos Gibbs Sampling e Metrópolis Hastings (ver por exemplo Gelfand \& Smith, 1990). Com esses métodos encontramos estimativas pontuais e por intervalo dos parâmetros de interesse do modelo.

O algoritmo Gibbs Sampling é um método de simulação via cadeias de Markov que fornece uma forma alternativa para se obter uma amostra da distribuição de interesse baseada em sucessivas gerações das distribuições condicionais (ver apêndice 1). Esse algoritmo é utilizado no caso em que as distribuições condicionais marginais são identificadas com uma distribuição conhecida.

Em modelos mais complexos, geralmente não temos formas conhecidas nas distribuições condicionais. Nesse caso, usamos o algoritmo Metropolis Hastings para a geração das amostras (ver apêndice 2).

A convergência da amostra à distribuição de equilibrio pode ser monitorada pelo método proposto por Gelman e Rubin (1992), (ver apêndice 3). 
Nos capitulos posteriores apresentamos uma análise Bayesiana utilizando os algoritmos Gibbs Sampling e Metropolis Hastings para os modelos utilizados para a resolução de alguns exemplos de aplicação em meta-análise.

Para alguns dos exemplos de aplicação introduzidos na Seção 1.3, utilizamos mais de um modelo para a análise dos dados, então um critério para seleção de modelos também foi aplicado.

\subsection{Algumas consideraçōes sobre seleção de modelos}

Quando mais de um modelo é considerado para analisar o conjunto de dados em questão, uma questão surge naturalmente: Qual ou quais modelos são mais apropriados para a análise dos dados?

Neste sentido, a metodologia para determinação de modelos se toma fundamental. Ela se divide em duas componentes: adequação do modelo e seleção do modelo.

Neste trabalho nos preocupamos em selecionar o melhor modelo dentre os estudados. A discussão relativa à escolha de modelos enfatiza as distribuições preditivas como elemento principal na comparação de modelos arbitrários.

Sob o enfoque Bayesiano, vários métodos para seleção de modelos estão propostos na literatura (ver por exemplo, Jeffreys, 1939; Box \& Hill, 1967; Geisser \& Edy, 1979; Gelfand \& Dey, 1994).

Um desses métodos é o que utiliza o fator de Bayes que consiste em calcular a razão de densidades a posteriori de dois modelos $M_{1}$ e $M_{2}$. A principal dificuldade do uso do fator de Bayes é a escolha de uma densidade a priori para cada um dos modelos. Quando essa escolha é tal que não dá preferência a um dos modelos, esse método coincide com o teste da razão de verossimilhança, caso contrário, mesmo utilizando densidades a priori não informativas, o fator de Bayes pode indicar um modelo inadequado devido o paradoxo de Lindley (ver por exemplo Press, S. J., 1989). Por essa razão, optamos pelo uso de densidades preditivas para a seleção de modelos.

\subsection{Distribuições preditivas}

A distribuição preditiva (ou verossimilhança marginal) é a distribuição marginal conjunta dos dados, que pode ser utiliżada de várias formas na seleção de modelos. 
Se $y=\left\{y_{1}, \ldots, y_{n}\right\}$ representa o conjunto das observações e $\theta=\left\{\theta_{1}, \ldots, \theta_{p}\right\}$ o conjunto de parâmetros do modelo, podemos considerar $p(\underset{\sim}{\mid} \mid \underline{\sim})$ representando a densidade conjunta das observações dado $\underset{\sim}{\theta}$ e $\pi(\theta)$ a densidade a priori de $\underset{\sim}{\theta}$, tendo $p(y \underline{\sim}(\underline{\theta}) \pi(\theta)$ como especificação do modelo, se $y$ e $\theta$ são supostos contínuos, podemos notar em geral, que a densidade preditiva pode ser definida por

$$
p(\underset{\sim}{y})=\int p(\underset{\sim}{y} \mid \underset{\sim}{\theta}) \pi(\underset{\sim}{\theta}) d \underline{\underline{\theta}}
$$

Se a distribuição a priori $\pi(\theta)$ for própria, não há nenhum problema na utilização de $p(y)$. Caso a priori seja imprópria, $p(y)$ não pode ser calculada pois a integral diverge. Neste caso, outras densidades sob diferentes justificativas podem ser usadas na discriminação de modelos.

Uma densidade preditiva muito utilizada é a densidade preditiva ordenada.

\subsubsection{Densidade preditiva condicional ordenada (CPO)}

A densidade preditiva para $y_{i}$ dado $y_{(i)}=\left(y_{1}, y_{2}, \ldots, y_{i-1}, y_{i+1}, \ldots, y_{n}\right)$ é dada por,

$$
\left.c_{i}=p\left(y_{i} \mid y_{(i)}\right)=\int p\left(y_{i} \mid \underline{\theta}\right) \pi \underset{\sim}{\theta} \underset{(i)}{y_{(i)}}\right) d \underset{\sim}{\theta}, \quad i=1,2, \ldots, n
$$

onde $\pi\left(\underset{\sim}{\theta} \mid y_{(i)}\right)$ é a densidade a posteriori para o vetor de parâmetros $\underset{\sim}{\theta}$, dado os dados $y_{(i)}$.

Usando o algoritmo Gibbs Sampling, é possivel aproximar (2.5) usando o estimador de Monte Carlo,

$$
\hat{p}\left(y_{i} \mid y_{(j)}\right)=\frac{l}{N} \sum_{j=1}^{N} p\left(y_{i} \mid \theta_{\sim}^{(j)}\right)
$$


onde $\theta_{\sim}^{(j)}, j=1,2, \ldots, N$, são as amostras geradas pelo amostrador de Gibbs.

\subsection{Escolha do modelo?}

Para a seleção de modelos, podemos utilizar a densidade preditiva $c_{l}=\hat{p}\left(y_{i} \mid y_{(1)}\right)$, examinando os valores dos $c_{i}$ para os diferentes modelos estudados, observando que o modelo mais adequado para os dados é o que apresenta valores maiores de $c_{i}$. Para isso, podemos construir gráficos de $c_{i}$ versus $i, i=1,2, \ldots, n$, para cada modelo, e o melhor modelo será aquele que apresentar a maioria dos valores de $c_{i}$ maiores entre os modelos comparados.

Uma forma alternativa é escolher o modelo tal que $c_{\ell}=\prod_{i=i}^{n} c_{i}(\ell)$ é o máximo, onde $\ell$ indica o modelo.

Além da técnica proposta acima, existem outras versões do fator de Bayes para discriminação de modelos (ver por exemplo, Jeffreys, 1961; Berger \& Pericchi, 1993; Aitkin, 1991).

\subsection{Verificação da adequação do modelo via densidade preditiva}

Não basta um modelo ser selecionado dentre outros, a verificação do ajuste do modelo é fundamental numa análise estatística. Vários métodos para verificar a adequação do ajuste e seleção de modelos são propostos na literatura (ver por exemplo, Gelman et al, 1995; Carlin \& Louis, 1997).

Neste trabalho usamos um procedimento para verificar a adequabilidade do modelo via densidade preditiva utilizando' a quantidade denominada nível descritivo Bayesiano ou "p-value" Bayesiano $\left(p_{v}\right)$. Neste sentido, podemos pensar que se o modelo está bem ajustado aos dados, então qualquer conjunto de dados gerado por este modelo deverá ser similar aos dados observados. Em outras palavras, os dados observados são plausíveis via densidade preditiva a posteriori. 


\subsubsection{Procedimento para verificação da adequação do modelo utilizando o nível descritivo Bayesiano $\left(p_{v}\right)$}

¿ste procedimento consiste em extrair réplicas da amostra através de valores simulados da distribuição preditiva a posteriori e comparar estas amostras replicadas com o conjunto de dados observados (a réplica seria o y que poderia ser observado futuramente se o experimento fosse repetido com o mesmo modelo e valor de $\theta$ que originou os dados atuais $(y)$ ). Qualquer diferença sistemática entre as amostras geradas (réplicas) e o conjunto de dados observados indicarão falhas do modelo ajustado.

\section{Nivel descritivo Bayesiano $\left(p_{\nu}\right)$}

O nível descritivo Bayesiano ( ou probabilidade caudal) é definido como a probabilidade que os dados replicados sejam mais extremos que os dados observados segundo uma estatística de teste $(T(y, \theta))$, ou seja,

$$
\begin{aligned}
& p_{v}=\operatorname{Prob}\left(T\left(y^{\text {rep }}, \theta\right) \geq T(y, \theta) \mid y\right) \\
& \iint \mathrm{I}_{\mathrm{T}\left(\mathrm{y}^{\text {re }}, \theta\right) \mathrm{r} r(y, \theta)} p\left(y^{\text {rep }}, \theta \mid y\right) d \theta d y^{\text {rep }} \\
& \iint \mathrm{I}_{\mathrm{T}\left(\mathrm{y}^{r e \theta}, \theta\right) \geq r(y, \theta)} \pi(\theta \mid y) p\left(y^{r e p} \mid \theta\right) d \theta d y^{\text {rep }}
\end{aligned}
$$

onde $y$ indica o vetor de dados observados;

$\theta$ : vetor de parâmetros;

$y^{\text {rep }}$ : cópia dos dados (réplica)

$p\left(y^{\text {rep }} \mid y\right)=\int p\left(y^{\text {rep }} \mid \theta\right) \pi(\theta \mid y) d \theta$ : densidade preditiva de $y^{\text {rep }} \mathrm{e}$

$T(y, \theta)$ : estatística do teste.

Devemos salientar que o nível descritivo Bayesiano $\left(p_{v}\right)$ não deve ser utilizado para a escolha de modelos, pois $p_{v}$ não indica a probabilidade que o modelo esteja correto dado os 
dados. Essa quantidade é útil para verificar a discrepância entre os dados observados e o modelo proposto.

Escolha de $T(y, \theta)$

A escolha de $T(y, \theta)$ depende do problema, em geral é escolhida com a finalidade de medir aspectos do dados que não são levados em consideração pelo modelo. Por exemplo, se temos interesse em verificar uma possivel assimetria da distribuição, $T(y, \theta)$ pode ser definida por,

$$
T(y \mid \theta)=\left|y_{(90)}-\theta\right|-\left|y_{(20)}-\theta\right|
$$

onde $y_{(90)}$ e $y_{(10)}$ correspondem, respectivamente, aos percentis 90 e 10 da distribuição.

Se quisermos verificar o ajuste do modelo para valores extremos da distribuição (por exemplo, no caso onde há suspeita de possíveis outliers no conjunto de dados), podemos utilizar,

$$
T(y \mid \theta)=\min _{l \leq i \leq n}\left\{y_{i}\right\} \text { ou } T(y \mid \theta)=\operatorname{móx}_{l \leq i \leq n}\left\{y_{i}\right\}
$$

Uma outra estatística de teste muito utilizada como alternativa as outras é definida por,

$$
T(y \mid \theta)=\sum_{i=1}^{n} \frac{\left(y_{i}-E\left(y_{i} \mid \theta\right)\right)^{2}}{\operatorname{Var}\left(y_{i} \mid \theta\right)}
$$

Notemos que a estatística de teste $T(y \mid \theta)$, na abordagem Bayesiana, pode ser função de parâmetros desconhecidos pois são obtidos através de simulações da distribuição a posteriori destes parâmetros. 
Procedimento para calcular $p_{v}$ via simulação

$1^{-}$passo: Gerar $\theta^{(\ell)}$ a partir da distribuição a posteriori $(\pi(\theta \mid y))$ e em seguida gerar $y^{\text {rep }(\ell)}$ via $p\left(y \mid \theta^{(\ell)}\right)$, para $\ell=1,2, \ldots, L$, onde $L$ indica o mímero de iterações.

$2^{-}$passo: Estimar $p_{v}$ da seguinte forma:

$$
\hat{p}_{\nu}=\frac{\#\left\{T\left(y^{\text {rep(l) }}, \theta^{(\ell)}\right) \geq T\left(y, \theta^{(\ell)}\right), \ell=1,2, \ldots, L\right\}}{L}
$$

onde o símbolo \#, aqui, indica o número de vezes que $T\left(y^{\text {rep( }(\ell)}, \theta^{(\ell)}\right)$ é maior ou igual a $T\left(y, \theta^{(\ell)}\right), \ell=1,2, \ldots, L$.

Após a obtenção da estimativa de $p_{v}$, podemos decidir pela rejeição do modelo se para uma determinada estatística de teste, $\hat{p}_{v}<0.05$ ou $\hat{p}_{v}>0.95$. Em outras palavras, o modelo estará bem ajustado quando o valor de $\hat{p}_{v}$ estiver em torno de 0.5 . Na prática, o modelo é aceitável se $\hat{p}_{v}$ estiver entre 0.2 e 0.8 , caso contrário, é preferível testar outros modelos. 


\section{CAPITULO 3 - ESTUDO SOBRE O USO DE UMA NOVA DROGA CONTRA EPILEPSIA}

\subsection{Introdução}

Em diversos estudos clínicos, muitas vezes, o interesse principal do pesquisador é comparar uma nova droga com uma tradicionalmente usada e observar se essa nova droga pode trazer sucesso na cura de uma determinada doença, ou, pelo menos, se essa nova droga pode ajudar a melhorar o estado clínico de um paciente com determinada doença. Pode-se estar interessado por exemplo na proporção de melhora que essa nova droga causa no estado clínico do paciente.

Para exemplificar este tipo de situação, será considerado um conjunto de dados introduzidos por Van Belle (1993) que traz 15 relatórios comparativos, independentes e agrupados de ensaios clínicos sobre uma nova droga antiepilética. A variável resposta foi definida como a redução acima de $50 \%$ na freqüência da doença no paciente.

A Tabela 1 apresenta o conjunto de dados introduzido por Van Belle. Na coluna 1 temos o número de cada estudo, na coluna 2 a ordem cronológica do estudo, na coluna 3 os totais de pacientes $n_{t}$ em cada estudo; na coluna 4 o número de pacientes que respondem ao tratamento, $y_{i}$, na coluna 5 a proporção observada de pacientes melhores $\left(\hat{p}_{i}\right)$ e na coluna 6 temos o logito $\hat{\theta}_{i}=\log \left(\frac{\hat{p}_{i}}{1-\hat{p}_{i}}\right)$. 
Tabela 1: Dados de 15 estudos independentes sobre a eficácia de uma nova droga antiepilética

\begin{tabular}{lcccccc}
\hline & Estudo & Original & $\boldsymbol{n}_{\boldsymbol{i}}$ & $\boldsymbol{y}_{\boldsymbol{i}}$ & $\hat{\mathbf{p}}_{\mathbf{i}}$ & $\hat{\boldsymbol{\theta}}_{\boldsymbol{i}}$ \\
\hline \multirow{3}{*}{ Estudos abertos } & 1 & 1 & 30 & 17 & 0.5666 & 0.2680 \\
& 2 & 7 & 16 & 8 & 0.5000 & 0.0000 \\
& 3 & 9 & 69 & 41 & 0.5942 & 0.3814 \\
& 4 & 10 & 23 & 13 & 0.5652 & 0.2623 \\
& 5 & 11 & 42 & 32 & 0.7619 & 1.1631 \\
& 6 & 15 & 151 & 90 & 0.5960 & 0.3888 \\
& & & & & & \\
& 7 & 2 & 5 & 0.2500 & -1.0986 \\
& 8 & 3 & 20 & 9 & 0.4500 & -0.2007 \\
& 9 & 4 & 17 & 3 & 0.1765 & -1.5402 \\
& 10 & 5 & 15 & 7 & 0.4667 & -1.1334 \\
& 11 & 6 & 18 & 8 & 0.4444 & -0.2233 \\
& 12 & 8 & 17 & 9 & 0.5294 & -0.1177 \\
& 13 & 12 & 19 & 1 & 0.0526 & -2.8910 \\
& 14 & 13 & 51 & 12 & 0.2353 & -1.1786 \\
& 15 & 14 & 59 & 17 & 0.2881 & -0.9046
\end{tabular}

Neste caso, onde temos interesse em comparar um novo tratamento com um tradicional, levando em conta $N$ estudos independentes, comparativos e agrupados, a resposta é do tipo dicotômica. Se o novo tratamento melhora o estado clínico do paciente em mais de $50 \%$ a resposta assume o valor 1 , caso contrário assume o valor zero. Desta forma podemos considerar um modelo de efeitos aleatórios agrupados, cuja resposta $y_{i}$ para cada estudo tem distribuição binomial com parâmetros $n_{i}$ e $p_{i}$.

O problema e o interesse do pesquisador foram definidos da seguinte forma:

Problema: Uma nova droga contra epilepsia esta sendo testada sob duas formas de tratamento: um tradicional (estudos fechados) e um novo (estudos abertos). Aqui, os estudos abertos representam um tratamento padrão utilizando a nova droga e os estudos fechados representam um tratamento com placebo. 


\section{Interesse do gesquisador:}

- Há um único valor para a proporção de pacientes respondendo ao tratamento? Ou podemos considerar as proporções observadas como amostras de uma população de proporções respondentes?

- Qual é a conclusão da melhoria geral pelo tratamento?

- É apropriado combinar as informações dos estudos?

- Há homogeneidade dentro de cada grupo?

- Uma média ponderada dos $\theta_{i}$ 's corresponde a presença de um efeito positivo do medicamento?

\subsection{Definição do modelo e suas respectivas variáveis}

Uma forma natural para descrever o problema e analisar os dados da Tabela 1 é o uso de um modelo Bayesiano hierárquico. $\mathrm{Na}$ análise Bayesiana hierárquica, os parâmetros das distribuições dos parâmetros, chamados hiperparâmetros, são desconhecidos mas com uma distribuição conhecida. Muitas vezes, um problema é estruturado de forma que existem relações probabilisticas a respeito dos parâmetros e hiperparâmetros que podem ser incorporadas ao modelo através de distribuições a priori hierárquicas. Desta forma temos,

$$
\begin{aligned}
& y_{i} \mid \theta_{i}, \mu, \delta_{k}, \sigma_{k}^{2} \stackrel{\text { ind }}{\sim} \operatorname{Binomial}\left(n_{i}, p_{i}\right) \\
& \theta_{i} \mid \mu, \delta_{k}, \sigma_{k}^{2} \sim \operatorname{Normal}\left(\mu+\delta_{k}, \sigma_{k}^{2}\right)
\end{aligned}
$$

onde: $y_{i}$ é o número de pacientes melhorados para o estudo $i, i=1,2, \ldots, N$;

$n_{i}$ é o número de pacientes que completaram o estudo $i, i=1,2, \ldots, N$;

$p_{i}$ é o parâmetro de percentagem de melhora para o estudo $i, i=1,2 \ldots N, 0>p_{i}>l \mathrm{e}$, $\theta_{i}=\log \left(\frac{p_{i}}{1-p_{i}}\right)$ 
Note que, $\theta_{i}$ é o logito estimado de melhora no i-ésimo estudo, $-\infty<\theta_{i}, \mu, \delta_{k}<\infty$;

$\sigma_{k}^{2}$ é a variância desta estimativa para o grupo $\mathrm{k}$, onde $\sigma_{k}^{2}>0$;

$\mu$ é a média geral e,

$\delta_{k}$ é o efeito aleatório para o grupo $k, k=1,2$,

onde, $k=l$ indica que os estudos são abertos e $k=2$ indica que os estudos são fechados.

A especificação binomial para a verossimilhança é razoável, já que as respostas são dicotômicas e os estudos são independentes. Modelos normais são convenientes para $\theta_{i}=\log \left(\frac{p_{i}}{l-p_{i}}\right)$ já que os logitos tem domínio definido em toda a reta real.

Agora, considere a estrutura da média e variância de $\theta_{1}$. Todos os estudos para os dados da Tabela 1 analisam a mesma droga, mas existem dois grupos distintos: alguns estudos são abertos e outros são fechados. Desta forma, parece razoável que a estrutura da média de $\theta_{i}$ contenha uma média geral $\mu$ mais um efeito aleatório específico para cada grupo $\delta_{k}, k=1,2$. Portanto, ajustamos os dados de cada grupo usando um modelo de efeitos aleatórios específico para cada grupo; daí o termo modelo de efeitos aleatórios agrupados. Tal bifurcação do conjunto de dados é comum em meta-análise onde, freqüentemente, deseja-se combinar, por exemplo, resultados de estudos controlados e não controlados ou estudos de caso-controle e coorte.

Uma posição conservadora é assumir que a variância de $\theta_{i}$ deve depender do grupo, pois não existe informação de que os dois grupos tenham a mesma variância.

Dentro de cada grupo é suposto permutabilidade do $\theta_{t}$ para descrever aspectos comuns entre os estudos e para evitar partições a priori dos dados. Portanto, para este problema, supor um modelo hierárquico da forma, 


$$
\begin{array}{lc}
y_{i}{ }^{\text {ind }} \operatorname{Binomial}\left(n_{i}, p_{i}\right), & 0<p_{i}<l, i=l, \ldots, N, \\
\theta_{i}=\log \left(\frac{p_{i}}{l-p_{i}}\right), & i=1, \ldots, N, \\
\theta_{i} \sim \text { Normal }\left(\mu+\delta_{k} ; \sigma_{k}^{2}\right), & -\infty<\theta_{i}<\infty, k=1,2 \\
\pi\left(\sigma_{k}^{2}\right) \sim \text { Gama imversa, } & \sigma_{k}^{2}>0, k=l, 2 \\
\pi(\mu) \sim \text { Normal, } & -\infty<\mu<\infty \\
\pi\left(\delta_{k}\right) \sim \text { Normal } & -\infty<\delta_{k}<\infty, k=1,2 .
\end{array}
$$

\subsubsection{Funçāo de verossimilhança}

Considerando o modelo sugerido em (3.1), temos que,

$$
y_{i} \stackrel{i \text { ind }}{\sim} \operatorname{Binomial}\left(n_{i}, p_{i}\right), \quad 0<p_{i}<l, i=l, \ldots, N
$$

Desta forma, a função de verossimilhança para $p_{1}, p_{2}, \ldots, p_{N}$ é dada por,

$$
L\left(p_{1}, \ldots, p_{N}\right)=\prod_{i=l}^{N}\left(\begin{array}{l}
n_{i} \\
y_{i}
\end{array}\right) p_{i}^{y_{i}}\left(l-p_{i}\right)^{n_{i}-y_{t}}
$$

como $\theta_{i}=\log \left(\frac{p_{i}}{1-p_{i}}\right)$, então $p_{i}=\frac{e^{\theta_{1}}}{1+e^{\theta_{i}}}$. Portanto, a função de verossimilhança para $\theta_{1}, \ldots, \theta_{N}$ é definida como, 


$$
\begin{aligned}
L\left(\theta_{1}, \ldots, \theta_{N}\right) & \propto \prod_{i=1}^{N}\left(\frac{e^{\theta_{i}}}{1+e^{\theta_{t}}}\right)^{y_{1}}\left(1-\frac{e^{\theta_{t}}}{l+e^{\theta_{1}}}\right)^{n_{t}-y_{1}}, \\
& =\prod_{i=1}^{N} \frac{e^{\theta_{i} y_{1}}}{\left(1+e^{\theta_{i}}\right)^{n_{1}}}
\end{aligned}
$$

sendo $N=N_{1}+N_{2}$, onde $N_{1}$ é o número de estudos abertos e $N_{2}$ o número de estudos fechados.

\subsubsection{Distribuiçāo a priori}

O modelo sugerido para uma análise inicial dos dados é um modelo proposto com as distribuições a priori especificadas abaixo:

$$
\begin{array}{lc}
\theta_{i} \mid \mu, \delta_{k}, \sigma_{k}^{2} \sim \operatorname{Normal}\left(\mu+\delta_{k}, \sigma_{k}^{2}\right) & -\infty<\theta_{i}<\infty \\
\pi\left(\sigma_{k}^{2}\right) \sim \operatorname{Gama} \operatorname{Inversa}\left(a_{k}, b_{k}\right) & \sigma_{k}^{2}>0, \quad k=1,2 \\
\pi(\mu) \sim \operatorname{Normal}(0, c) & -\infty<\mu<\infty \\
\pi\left(\delta_{k}\right) \sim \operatorname{Normal}(0, d) & -\infty<\delta_{k}<\infty, \quad k=1,2
\end{array}
$$

onde, para o conjunto de dados da Tabela 1, consideramos $a_{k}=0.001, b_{k}=1000, c=10$ e $d=10$.

Para a distribuição a priori de $\sigma_{k}^{2}, k=1,2$, usamos uma distribuição Gama-inversa escolhendo um valor de $a$ pequeno e um valor de $b$ grande de forma a assegurar as propriedades da distribuição a posteriori.

As distribuições a priori para $\mu e \delta_{k}, k=l, 2$, foram escolhidas como sendo distribuições a priori normais com variância suficientemente grandes por falta de conhecimento a priori. 
Alguém pode sustentar que o valor de $a$ e $b$ iguais a 10 para a variância em $\pi(\mu)$ e $\pi\left(\delta_{k}\right), k=1,2$ não é suficientemente grande, porém, considere que este valor reflete a suposição que a média de $\theta_{1}$ quase sempre encontra-se no intervalo $(0 \pm 3 \sqrt{10+10})=(-13.42 ; 13.42)$ e, portanto, a média de $p_{1}$ quase sempre encontra-se no intervalo $\left[e^{-13.42} /\left(1+e^{-13.42}\right) ; e^{13.42} /\left(1+e^{13.42}\right)\right]=(0.0000015 ; 0.9999985)$, o qual é certamente razoável supor na prática.

Assumindo um valor mais alto para a variância (por exemplo, $a$ e $b$ iguais a 25), os valores foram muito próximos aos obtidos com os valores de $a$ e $b$ iguais a 10

Partindo de (3.6) a distribuição a priori para $\theta_{1}, \ldots, \theta_{N_{1}}$ é dada por,

$$
\begin{aligned}
\pi\left(\theta_{1}, \ldots, \theta_{N_{1}}\right) & =\prod_{i=1}^{N_{1}} \frac{1}{(2 \pi)^{1 / 2}\left(\sigma_{1}^{2}\right)^{1 / 2}} \exp \left\{-\frac{1}{2 \sigma_{1}^{2}}\left(\theta_{i}-\mu-\delta_{1}\right)^{2}\right\} \\
& \propto \frac{1}{\left(\sigma_{1}^{2}\right)^{N_{1} / 2}} \exp \left\{-\frac{1}{2 \sigma_{1}^{2}}\left(\theta_{i}-\mu-\delta_{1}\right)^{2}\right\}
\end{aligned}
$$

e a distribuição a priori para $\theta_{N_{1}+1}, \ldots, \theta_{N}$ é dada por,

$$
\begin{aligned}
\pi\left(\theta_{N_{1}+1}, \ldots, \theta_{N}\right)= & \prod_{i=N_{1}+1}^{N} \frac{1}{(2 \pi)^{1 / 2}\left(\sigma_{2}^{2}\right)^{1 / 2}} \exp \left\{-\frac{1}{2 \sigma_{2}^{2}}\left(\theta_{i}-\mu-\delta_{2}\right)^{2}\right\} \\
& \propto \frac{1}{\left(\sigma_{2}^{2}\right)^{N_{2} / 2}} \exp \left\{-\frac{1}{2 \sigma_{2}^{2}}\left(\theta_{i}-\mu-\delta_{2}\right)^{2}\right\}
\end{aligned}
$$

As distribuiçōes a priori para $\sigma_{1}^{2}, \sigma_{2}^{2}, \mu, \delta_{1}$ e $\delta_{2}$ são definidas, respectivamente, por,

$$
\pi\left(\sigma_{1}^{2}\right) \propto\left(\sigma_{1}^{2}\right)^{-\left(a_{1}+1\right)} \exp \left\{-\frac{1}{b_{1}\left(\sigma_{1}^{2}\right)}\right\}
$$




$$
\begin{aligned}
& \pi\left(\sigma_{2}^{2}\right) \propto\left(\sigma_{2}^{2}\right)^{-\left(a_{2}+1\right)} \exp \left\{-\frac{1}{b_{2}\left(\sigma_{2}^{2}\right)}\right\} \\
& \pi(\mu) \propto \exp \left\{-\frac{\mu^{2}}{2 c}\right\} ; \\
& \pi\left(\delta_{1}\right) \propto \exp \left\{-\frac{\delta_{1}^{2}}{2 d}\right\} \\
& \pi\left(\delta_{2}\right) \propto \exp \left\{-\frac{\delta_{2}^{2}}{2 d}\right\} .
\end{aligned}
$$

Portanto, assumindo independência a priori, a distribuição a priori conjunta para $\theta_{1}, \theta_{2}, \ldots, \theta_{N}, \sigma_{1}^{2}, \sigma_{2}^{2}, \mu, \delta_{1}$ e $\delta_{2}$ é dada por,

$$
\begin{aligned}
& \pi\left(\theta_{1}, \ldots, \theta_{N}, \sigma_{1}^{2}, \sigma_{2}^{2}, \mu, \delta_{1}, \delta_{2}\right) \propto\left(\sigma_{1}^{2}\right)^{-N_{1} / 2} \exp \left\{-\frac{1}{2 \sigma_{1}^{2}} \sum_{i=1}^{N_{1}}\left(\theta_{i}-\mu-\delta_{l}\right)^{2}\right\} \times \\
& \times\left(\sigma_{2}^{2}\right)^{-N_{2} / 2} \exp \left\{-\frac{1}{2 \sigma_{2}^{2}} \sum_{l=N_{1}+1}^{N}\left(\theta_{i}-\mu-\delta_{2}\right)^{2}\right\} \times\left(\sigma_{1}^{2}\right)^{-\left(a_{1}+1\right)} \times\left(\sigma_{2}^{2}\right)^{-\left(a_{2}+1\right)} \times \\
& \quad \times \exp \left\{-\frac{1}{b_{1} \sigma_{1}^{2}}-\frac{1}{b_{2} \sigma_{2}^{2}}-\frac{\mu^{2}}{2 c}-\frac{\delta_{1}^{2}}{2 d}-\frac{\delta_{2}^{2}}{2 d}\right\}
\end{aligned}
$$

\subsubsection{Distribuição a posteriori conjunta}

Combinando a distribuição a priori conjunta definida em (3.14) com a função de verossimilhança dada em (3.5), a distribuição a posteriori conjunta é dada por, 


$$
\begin{aligned}
& \left.\left.\pi\left(\theta_{1}, \ldots, \theta_{N}, \sigma_{1}^{2}, \sigma_{2}^{2}, \mu, \delta_{1}, \delta_{2} \mid \text { Dados }\right) \propto \prod_{i=1}^{N}\left[e^{\theta_{1} y_{1}}\left(I+e^{\theta_{1}}\right)^{-n_{1}}\right] \times\left(\sigma_{1}^{2}\right)^{-\left(\frac{N_{1}}{2}+a_{1}+1\right.}\right) \times\left(\sigma_{2}^{2}\right)^{-\left(\frac{N_{2}}{2}+a_{2}+1\right.}\right)_{\times} \\
& \times \exp \left\{-\frac{l}{2 \sigma_{1}^{2}} \sum_{i=1}^{N_{1}}\left(\theta_{i}-\mu-\delta_{1}\right)^{2}\right\} \times \exp \left\{-\frac{1}{2 \sigma_{2}^{2}} \sum_{i=N_{1}+1}^{N}\left(\theta_{i}-\mu-\delta_{2}\right)^{2}\right\} \times \\
& \times \exp \left\{-\frac{1}{b_{1} \sigma_{1}^{2}}-\frac{1}{b_{2} \sigma_{2}^{2}}-\frac{\mu^{2}}{2 c}-\frac{\delta_{1}^{2}}{2 d}-\frac{\delta_{2}^{2}}{2 d}\right\}
\end{aligned}
$$

Lembrando que $N_{1}$ é o número de estudos abertos e $N_{2}$ é o número de estudos fechados, a partir da distribuição a posteriori obtida, várias questões de interesse podem ser analisadas de forma direta, usando a formulação hierárquica Bayesiana.

\subsection{Aspectos computacionais}

Com o desenvolvimento de métodos baseados em simulação MCMC (Monte Carlo em Cadeias de Markov) tem sido possivel a análise de densidades a posteriori muito complicadas e até mesmo intratáveis. Para esse problema, utilizamos os algoritmos Metropolis-Hastings com Gibbs Sampling (ver alguns detalhes computacionais nos Apêndices).

Usamos o algoritmo Gibbs Sampling (ver Gelfand \& Smith, 1990) para gerar amostras das distribuições condicionais completas, as quais são encontradas como sendo:

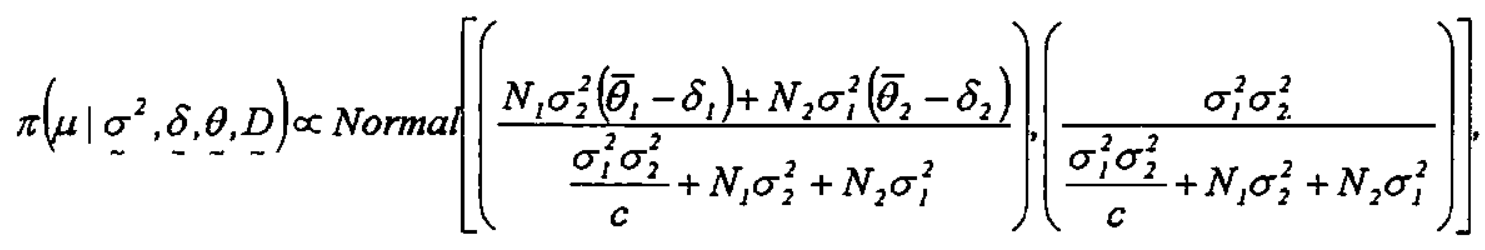

$$
\begin{aligned}
& \pi\left(\delta_{k} \mid \mu, \sigma_{\sim}^{2}, \underset{\sim}{\theta}, \underset{\sim}{\sim}\right) \propto \text { Normal }\left[\left(\frac{N_{k}\left(\bar{\theta}_{k}-\mu\right)}{\frac{\sigma_{k}^{2}}{d}+N_{k}}\right),\left(\frac{\sigma_{k}^{2}}{\frac{\sigma_{k}^{2}}{d}+N_{k}}\right)\right],
\end{aligned}
$$




$$
\pi\left(\sigma_{k}^{2} \mid \mu, \underset{\sim}{\delta}, \underset{\sim}{D}\right) \propto \text { Gama-Inversa }\left[\left(\frac{N_{k}}{2}+a_{k}\right),\left(\left(\frac{1}{b_{k}}+\frac{1}{2} \sum_{i=1}^{N_{2}}\left(\theta_{1}-\left(\mu+\delta_{k}\right)\right)^{2}\right)^{-1}\right)\right]
$$

e usamos o algoritmo Metropolis-Hastings (ver Chib \& Greenberg, 1995) para gerar as distribuições condicionais que não foram identificadas com uma distribuição conhecida, como é o caso das condicionais para $\theta_{1}, i=1, \ldots, N$. Assim, temos que:

$$
\pi\left(\theta_{i} \mid \mu, \underset{\sim}{\delta}, \sigma^{2}, \theta_{(i)}, \underset{\sim}{D}\right) \propto e^{\theta_{i} y_{t}-\frac{\left(\theta_{1}-\left(\mu+\delta_{2}\right)\right)^{2}}{2 \sigma_{2}^{2}}}\left(1+e^{\left.\theta_{l}\right)^{-n t}}\right.
$$

Para o uso do algoritmo Metropolis-Hastings, consideramos o núcleo para $\theta_{i}$ como sendo uma distribuição Normal, assim temos:

$$
\pi(\theta_{i}, \mu, \underset{\sim}{\delta} \underline{\sim}^{2}, \underbrace{}_{(i)}, \underset{\sim}{D}) \propto \operatorname{Normal}\left(\mu+\delta_{k}, \sigma_{k}^{2}\right) \times \psi\left(\theta_{i}\right)
$$

onde

$$
\begin{aligned}
& \psi\left(\theta_{i}\right)=\exp \left\{\theta_{i} y_{i}-n_{i} \ln \left(1+e^{\theta_{1}}\right)\right\} \\
\underline{\theta}= & \left(\theta_{1}, \ldots, \theta_{N}\right), \theta_{(i)}=\left(\theta_{1}, \ldots, \theta_{i-1}, \theta_{i+1}, \ldots, \theta_{N}\right), \sigma^{2}=\left(\sigma_{1}^{2}, \sigma_{2}^{2}\right), \underset{\sim}{\delta}=\left(\delta_{1}, \delta_{2}\right), \underset{\sim}{D}=\left(y_{1}, \ldots, y_{N}\right) \\
\bar{\theta}_{k}= & \frac{\sum_{i=1}^{N_{2}} \theta_{i}}{N_{k}}, k=1,2 \text { e } N=N_{1}+N_{2}
\end{aligned}
$$

e os valores de $c$ e $d$ se referem à variância a priori de $\mu$ e $\delta_{k}$ respectivamente.

No decorrer dos estudos fomos surpreendidos com um problema na parametrização original que não levava a resultados satisfatórios. Em particular, o problema estava na convergência dos valores referentes a $\mu \mathrm{e} \delta_{k}$. Os valores para cada um destes parâmetros convergiam para valores diferentes a cada simulação realizada, mas a soma deles $\left(\mu+\delta_{l}\right.$ e 
$\mu+\delta_{2}$ ), que se refere a média do parâmetro de interesse $\theta_{i}$, sempre convergiam para o mesmo valor. Então, uma reparametrização foi realizada.

\subsection{Uma reparametrização}

A reparametrização realizada foi a seguinte:

$$
\xi_{k}=\mu+\delta_{k} \quad k=1,2
$$

Utilizando esta reparametrização, a distribuição a priori conjunta é dada por:

$$
\begin{aligned}
& \pi\left(\theta_{1}, \ldots, \theta_{N}, \sigma_{l}^{2}, \sigma_{2}^{2}, \xi_{l}, \xi_{2}\right) \propto\left(\sigma_{l}^{2}\right)^{-N_{1} / 2} \exp \left\{-\frac{1}{2 \sigma_{l}^{2}} \sum_{l=1}^{N_{1}}\left(\theta_{1}-\xi_{l}\right)^{2}\right\} \times . \\
& \times\left(\sigma_{2}^{2}\right)^{-N_{2} / 2} \exp \left\{-\frac{1}{2 \sigma_{2}^{2}} \sum_{i=N_{1}+1}^{N}\left(\theta_{1}-\xi_{2}\right)^{2}\right\} \times\left(\sigma_{l}^{2}\right)^{-\left(a_{1}+1\right)} \times\left(\sigma_{2}^{2}\right)^{-\left(a_{2}+1\right)} \times . \\
& \times \exp \left\{-\frac{1}{b_{1} \sigma_{l}^{2}}-\frac{1}{b_{2} \sigma_{2}^{2}}-\frac{\xi_{l}^{2}}{2 c}-\frac{\xi_{2}^{2}}{2 d}\right\}
\end{aligned}
$$

A função de verossimilhança para $\theta_{1}, \ldots, \theta_{N}$ é a mesma dada por (3.5), ou seja:

$$
L\left(\theta_{1}, \ldots, \theta_{N}\right) \propto \prod_{i=l}^{N} \frac{e^{\theta_{i} y_{l}}}{\left(1+e^{\theta_{1}}\right)^{y_{1}}}
$$

e distribuição a posteriori conjunta ficou definida como, 


$$
\begin{aligned}
\left.\left.\pi\left(\theta_{1}, \ldots, \theta_{N}, \sigma_{1}^{2}, \sigma_{2}^{2}, \xi_{1}, \xi_{2} \mid D\right) \propto \prod_{i=1}^{N}\left[e^{\theta_{1} y_{1}}\left(I+e^{\theta_{l}}\right)^{-n_{l}}\right] \times\left(\sigma_{l}^{2}\right)^{-\left(\frac{N_{1}}{2}+a_{3}+1\right.}\right) \times\left(\sigma_{2}^{2}\right)^{-\left(\frac{N_{2}}{2}+a_{2}+1\right.}\right)_{\times} \\
\times \exp \left\{-\frac{1}{2 \sigma_{1}^{2}} \sum_{i=1}^{N_{1}}\left(\theta_{i}-\xi_{1}\right)^{2}\right\} \times \exp \left\{-\frac{1}{2 \sigma_{2}^{2}} \sum_{i=N_{l}+1}^{N}\left(\theta_{i}-\xi_{2}\right)^{2}\right\} \times \\
\\
\times \exp \left\{-\frac{1}{b_{1} \sigma_{1}^{2}}-\frac{1}{b_{2} \sigma_{2}^{2}}-\frac{\xi_{1}^{2}}{2 c}-\frac{\xi_{2}^{2}}{2 d}\right\}
\end{aligned}
$$

A partir da distribuição a posteriori (3.24) obtida através da reparametrização, as distribuições condicionais marginais são dadas por,

$$
\begin{aligned}
& \pi\left(\xi_{i} \mid \underline{\sigma}^{2}, \underset{\sim}{\theta} \underset{\sim}{D}\right) \propto \text { Normal }\left[\left(\frac{\frac{\sigma_{i}^{2}}{2 c}+N_{l} \bar{\theta}_{l}}{\frac{\sigma_{l}^{2}}{c}+N_{i}}\right),\left(\frac{\sigma_{l}^{2}}{\frac{\sigma_{l}^{2}}{c}+N_{i}}\right)\right] \\
& \pi\left(\xi_{2} \mid \underset{\sim}{\sigma^{2}}, \underset{\sim}{\theta} \underset{\sim}{D}\right) \propto N o r m a l\left[\left(\frac{\frac{\sigma_{2}^{2}}{2 d}+N_{2} \bar{\theta}_{2}}{\frac{\sigma_{2}^{2}}{d}+N_{2}}\right),\left(\frac{\sigma_{2}^{2}}{\frac{\sigma_{2}^{2}}{d}+N_{2}}\right)\right] \\
& \pi\left(\sigma_{k}^{2} \mid \underset{\sim}{\xi} \underset{\sim}{\theta, D}\right) \propto G \text { Gama }- \text { Inversa }\left[\left(\frac{N_{k}}{2}+a_{k}\right),\left(\left(\frac{1}{b_{k}}+\frac{1}{2} \sum_{i=1}^{N_{k}}\left(\theta_{i}-\left(\xi_{k}\right)\right)^{2}\right)^{-l}\right)\right] \\
& \pi\left(\theta_{i} \mid \underset{\sim}{\xi}, \sigma_{\sim}^{2}, \theta_{(i)}, \underset{\sim}{D}\right) \propto e^{\theta_{i} y_{i}-\frac{\left(\theta_{t}-\left(\xi_{t}\right)\right)^{2}}{2 \sigma_{i}^{2}}}\left(1+e^{\theta_{1}}\right)^{-\pi i}
\end{aligned}
$$

Mesmo com a reparametrização, não conseguimos identificar a distribuição marginal condicional de $\theta_{i}$ com uma distribuição conhecida. Por esta razão, o algoritmo MetropolisHastings para gerar os $\theta_{i}$ 's foi novamente empregado. $\mathrm{O}$ núcleo considerado para $\theta_{i}$ continua sendo uma distribuição Normal, asşim temos: 


$$
\pi\left(\theta_{i}, \underline{\sim}^{\sigma^{2}}, \sigma_{(i)}, \underline{-}\right) \propto \operatorname{Normal}\left(\xi_{k}, \sigma_{k}^{2}\right) \times \psi\left(\theta_{i}\right)
$$

onde $\psi\left(\theta_{i}\right)$ continua sendo dado por,

$$
\psi\left(\theta_{i}\right)=\exp \left\{\theta_{i} y_{i}-n_{i} \ln \left(1+e^{\theta_{i}}\right)\right\}
$$

e,

$$
\begin{aligned}
& \underline{\theta}=\left(\theta_{1}, \ldots, \theta_{N}\right), \underset{\sim}{\theta_{(i)}}=\left(\theta_{1}, \ldots, \theta_{i-l}, \theta_{i+l}, \ldots, \theta_{N}\right), \underline{\sigma}^{2}=\left(\sigma_{l}^{2}, \sigma_{2}^{2}\right), \underset{\sim}{\xi}=\left(\xi_{1}, \xi_{2}\right), \underline{D}=\left(y_{1}, \ldots, y_{N}\right) \\
& \bar{\theta}_{k}=\frac{\sum_{i=1}^{N_{1}} \theta_{i}}{N_{k}}, k=1,2 \text { e } N=N_{1}+N_{2}
\end{aligned}
$$

e os valores de $c$ e $d$ se referem a variância a priori de $\xi_{1} e \xi_{2}$ respectivamente.

\subsection{Resultados}

A partir da reparametrização considerada, conseguimos resultados mais satisfatórios. Daí, temos respostas às questões formuladas na Seção 3.1 .

A Tabela 2 sumariza os resultados obtidos via simulação para cada um dos parâmetros. Os estimadores Bayesianos obtidos (médias a posteriori) são estimadores de Monte Carlo baseados nas amostras geradas pelos algoritmos Gibbs Sampling e Metropolis-Hastings. 
Tabela 2: Média a posteriori e intervalos com $95 \%$ de credibilidade para cada um dos parâmetros do modelo

\begin{tabular}{ccc}
\hline Parâmetros & Médias a posteriori & Intervalos de credibilidade (95\%) \\
\hline$\xi_{1}$ & 0.4499 & $(-0.1047 ; 1.0002)$ \\
$\xi_{2}$ & -0.8396 & $(-1.7487 ; 0.0762)$ \\
$\sigma_{1}^{2}$ & 0.0575 & $(0.0007 ; 0.3804)$ \\
$\sigma_{2}^{2}$ & 0.3313 & $(0.0012 ; 1.6004)$ \\
$p_{1}$ & 0.5962 & $(0.4697 ; 0.7125)$ \\
$p_{2}$ & 0.5807 & $(0.3963 ; 0.7082)$ \\
$p_{3}$ & 0.6009 & $(0.5062 ; 0.6969)$ \\
$p_{4}$ & 0.5960 & $(0.4606 ; 0.7149)$ \\
$p_{5}$ & 0.6721 & $(0.5573 ; 0.8137)$ \\
$p_{6}$ & 0.6017 & $(0.5298 ; 0.6703)$ \\
$p_{7}$ & 0.2729 & $(0.1283 ; 0.4359)$ \\
$p_{8}$ & 0.3973 & $(0.2365 ; 0.5949)$ \\
$p_{9}$ & 0.2333 & $(0.0860 ; 0.3999)$ \\
$p_{10}$ & 0.4003 & $(0.2242 ; 0.6261)$ \\
$p_{11}$ & 0.3892 & $(0.2195 ; 0.6017)$ \\
$p_{12}$ & 0.4386 & $(0.2560 ; 0.6539)$ \\
$p_{13}$ & 0.1599 & $(0.0273 ; 0.3307)$ \\
$p_{14}$ & 0.2531 & $(0.1493 ; 0.3661)$ \\
$p_{15}$ & 0.2913 & $(0.1918 ; 0.4000)$ \\
\hline
\end{tabular}

Analisando a média a posteriori para $\sigma_{1}^{2} e \sigma_{2}^{2}$, podemos perceber que $\sigma_{2}^{2}>\sigma_{1}^{2}$, indicando que os estudos abertos formam um grupo mais homogêneo do que os estudos fechados. Desta forma, uma afirmação positiva a respeito de existir um único valor para a proporção de pacientes melhorados seria errada.

$\mathrm{O}$ quadro abaixo mostra as médias a posteriori ponderadas para os $p_{i}{ }^{\prime} s$.

\begin{tabular}{|c|c|c|}
\hline$M . P_{\text {geral }}$ & M.P $P_{\text {abertos }}$ & $M . P_{\text {fechados }}$ \\
\hline 0.4804 & 0.6086 & 0.3007 \\
\hline
\end{tabular}


onde $M . P_{\text {gerai }}=\frac{\sum_{i=1}^{N} n_{i} p_{i}}{\sum_{i=1}^{N} n_{i}}$ se refere a média a posteriori ponderada de $p_{i}$ para todos os estudos
$M . P_{\text {aberas }}=\frac{\sum_{i=1}^{N_{1}} n_{i} p_{i}}{\sum_{i=1}^{N_{1}} n_{i}}$ é a média a posteriori ponderada somente para os estudos abertos e
$M . P_{\text {jechados }}=\frac{\sum_{i=N_{t}+1}^{N} n_{i} p_{i}}{\sum_{i=N_{1}+1}^{N} n_{l}}$ é a média a posteriori ponderada somente para os estudos fechados.

Em cada caso, o peso de um estudo equivale ao tamanho amostral dele.

Através da média ponderada geral $\left(M . P_{\text {geral }}\right)$, podemos concluir que quando todos os estudos são analisados conjuntamente, não existe provas da eficácia do medicamento, ou seja, não existe provas de que a administração do medicamento fornece uma melhora significativa na resposta do paciente sobre o tratamento tradicional, lembrando que a resposta é significativa se o novo medicamento proporciona uma redução superior a $50 \%$ na freqüência da doença nos pacientes.

Observando as médias a posteriori dos $p_{i}{ }^{\prime} s$ dadas na Tabela 3 , verificamos que para todos os estudos abertos $\left(p_{1}, \ldots, p_{6}\right)$ as médias a posteriori estão entre 0.58 e 0.68 , e para estudos fechados $\left(p_{7}, \ldots, p_{15}\right)$ estão entre 0.15 e 0.44 , significando que os estudos abertos sustentam a eficácia do medicamento, enquanto os estudos fechados sustentam a hipótese contrária.

Sendo assim, não é apropriado combinar as informações dos estudos. Também o fato das médias a posteriori para os estudos fechados se apresentarem mais espalhadas $(0.15 ; 0.44)$, enquanto para os estudos abertos se apresentam mais concentradas $(0.58 ; 0.68)$, é um grande indicativo para concluirmos que os estudos abertos formam um grupo mais homogêneo ao contrário dos estudos fechados. 
A diferença entre estudos abertos e fechados também pode ser verificada através de uma estimativa da média populacional para $\theta_{i}$ dada por $\xi_{k}=\mu+\delta_{k}, k=1,2$, ou seja, o $p_{i}$ para os estudos abertos se reduz à $\frac{e^{a+499}}{l+e^{a .499}}=0.6106$ e para os estudos fechados, o $p_{i}$ se reduz à $\frac{e^{-0.8396}}{l+e^{-0.8396}}=0.3016$ como o esperado, onde 0.4499 é a média a posteriori de $\mu+\delta_{1}$ e $(-0.8396)$ é a média a posteriori de $\mu+\delta_{2}$.

Com esses resultados notamos que a média a posteriori $\xi_{k}=\mu+\delta_{k}$ em relação a média ponderada estão bem próximas, podendo concluir mais uma vez que os estudos abertos sustentam a eficácia do medicamento enquanto os estudos fechados sustentam uma hipótese contrária.

\subsubsection{Análise gráfica}

Uma análise gráfica é de extrema importância para a visualização dos resultados obtidos e, sem dúvida, de grande valia para a análise e interpretação dos mesmos.

As Figura 1 mostra a forma da distribuição marginal a posteriori para cada um dos parâmetros. 

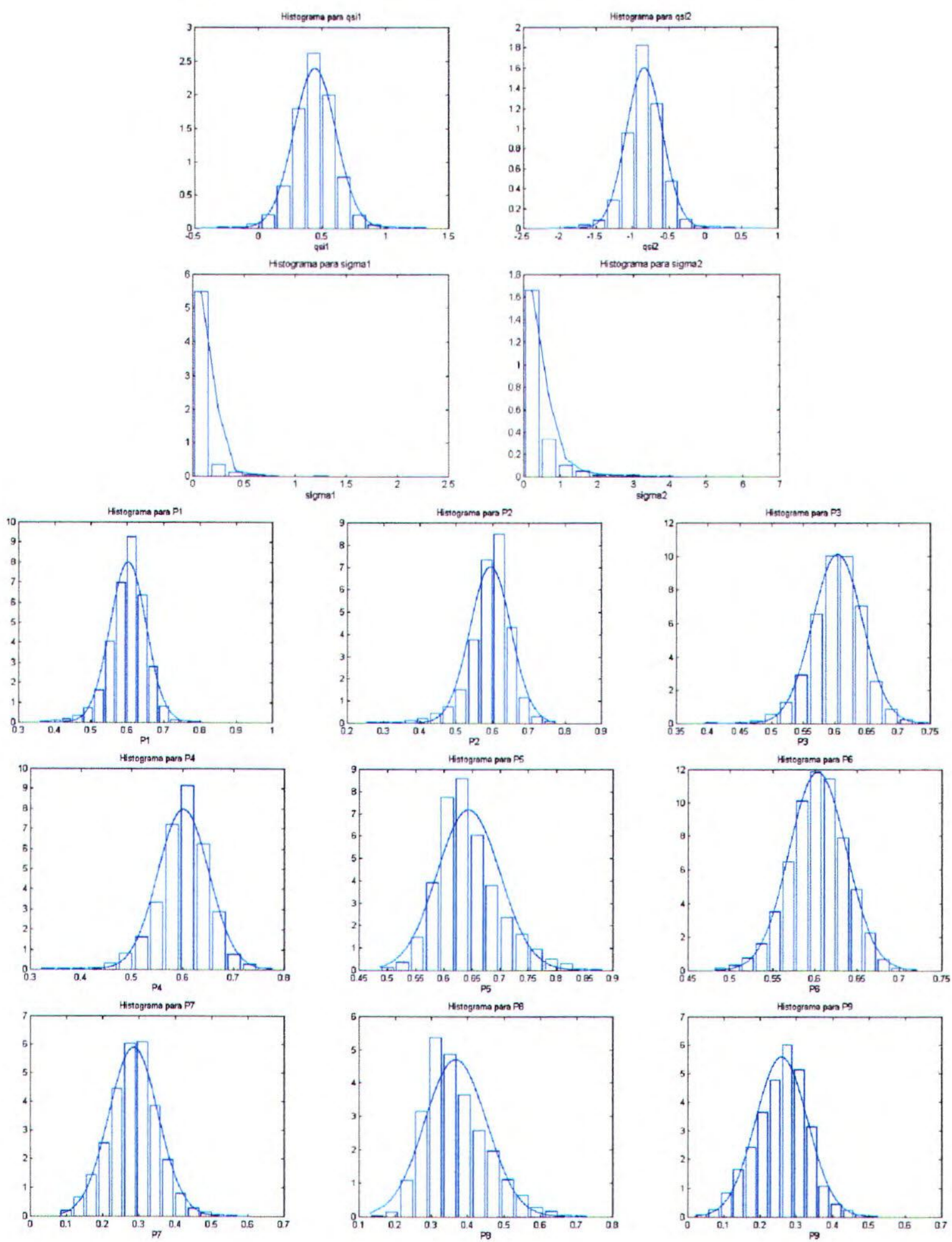

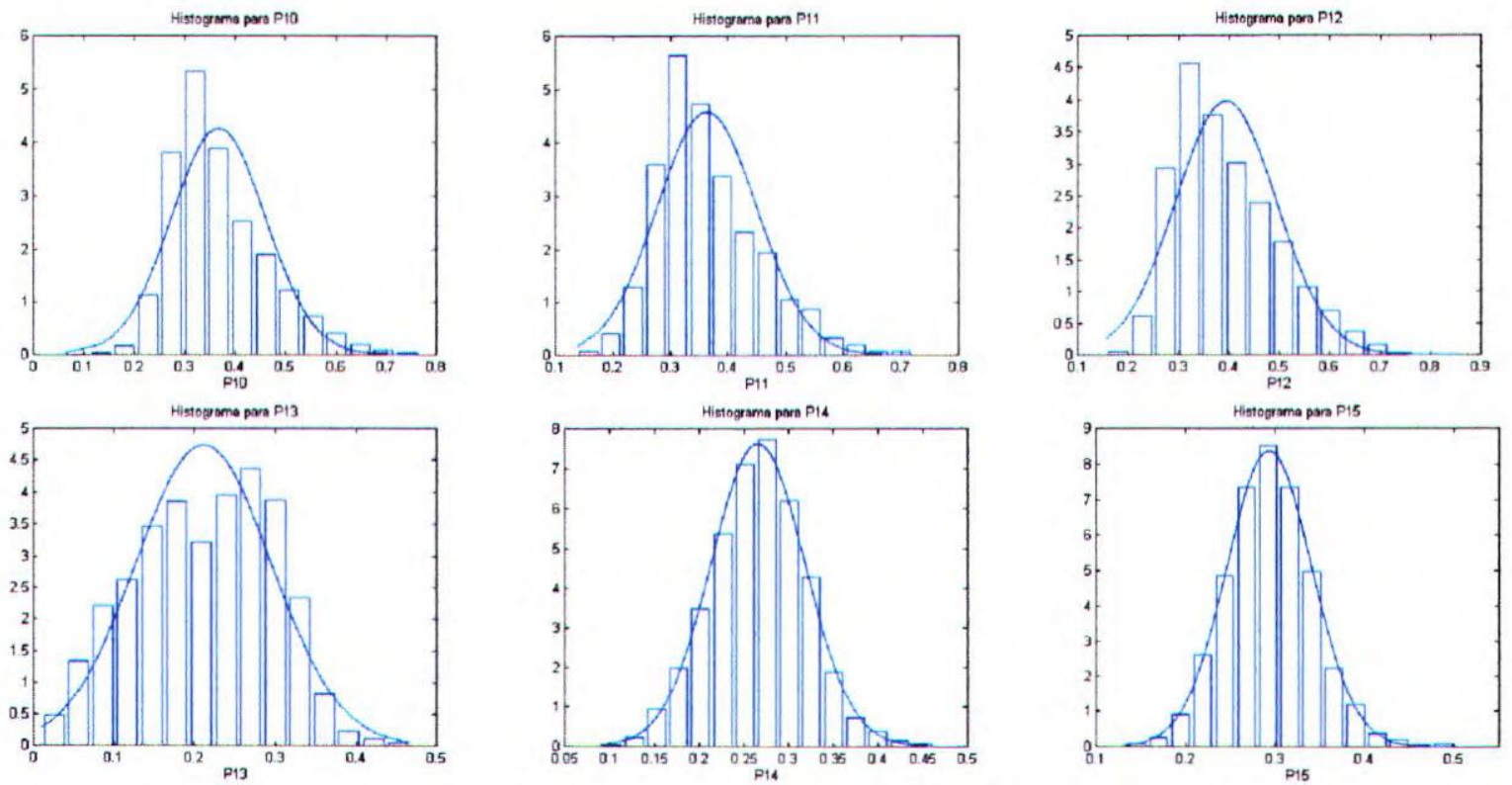

Figura 1: Densidades a posteriori marginais para $\xi_{1}, \xi_{2}, \sigma_{1}^{2}, \sigma_{2}^{2}, p_{i}, i=1,2, \ldots, 15$, respectivamente.

Sobrepondo estes gráficos, podemos tirar conclusões graficamente analisando o deslocamento das médias

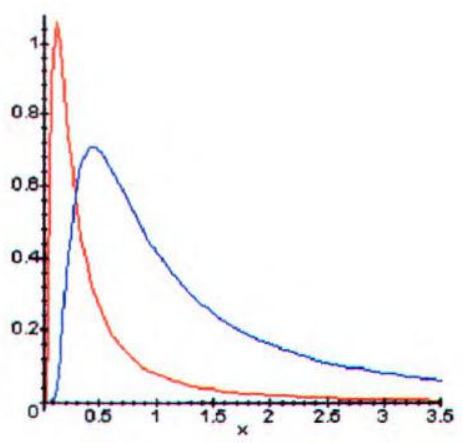

Figura 2: Densidades a posteriori para $\sigma_{1}^{2}$ (estudos abertos: linha vermelha) e $\sigma_{2}^{2}$ (estudos fechados: linha azul). 


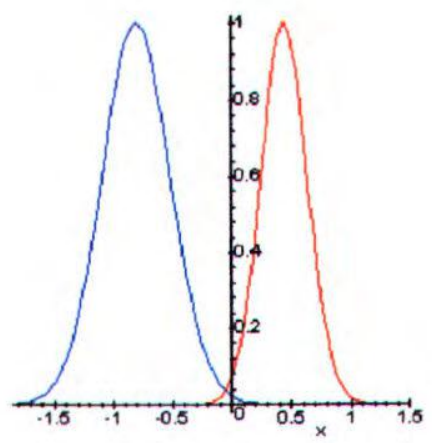

Figura 3: Densidades a posteriori para $\xi_{1}$ (estudos abertos: linha vermelha) e $\xi_{2}$ (estudos fechados: linha azul).

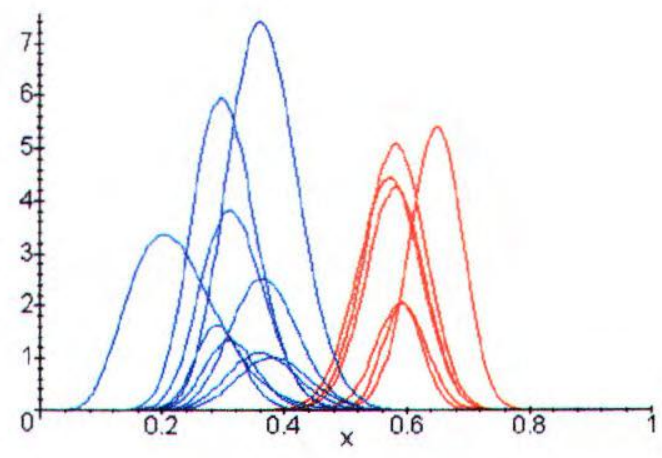

Figura 4: Densidades a posteriori para $p_{i}, i=1, \ldots, 15$ (estudos abertos: linhas vermelhas e estudos fechados: linhas azuis).

A Figura 4 mostra o agrupamento dos estudos fechados de um lado e abertos do outro, confirmando a diferença significativa que existe entre eles.

Logo abaixo, a Figura 5, mostra graficamente a convergência dos parâmetros que foi confirmada através do critério de convergência apresentados por Gelman e Rubin (1992). 

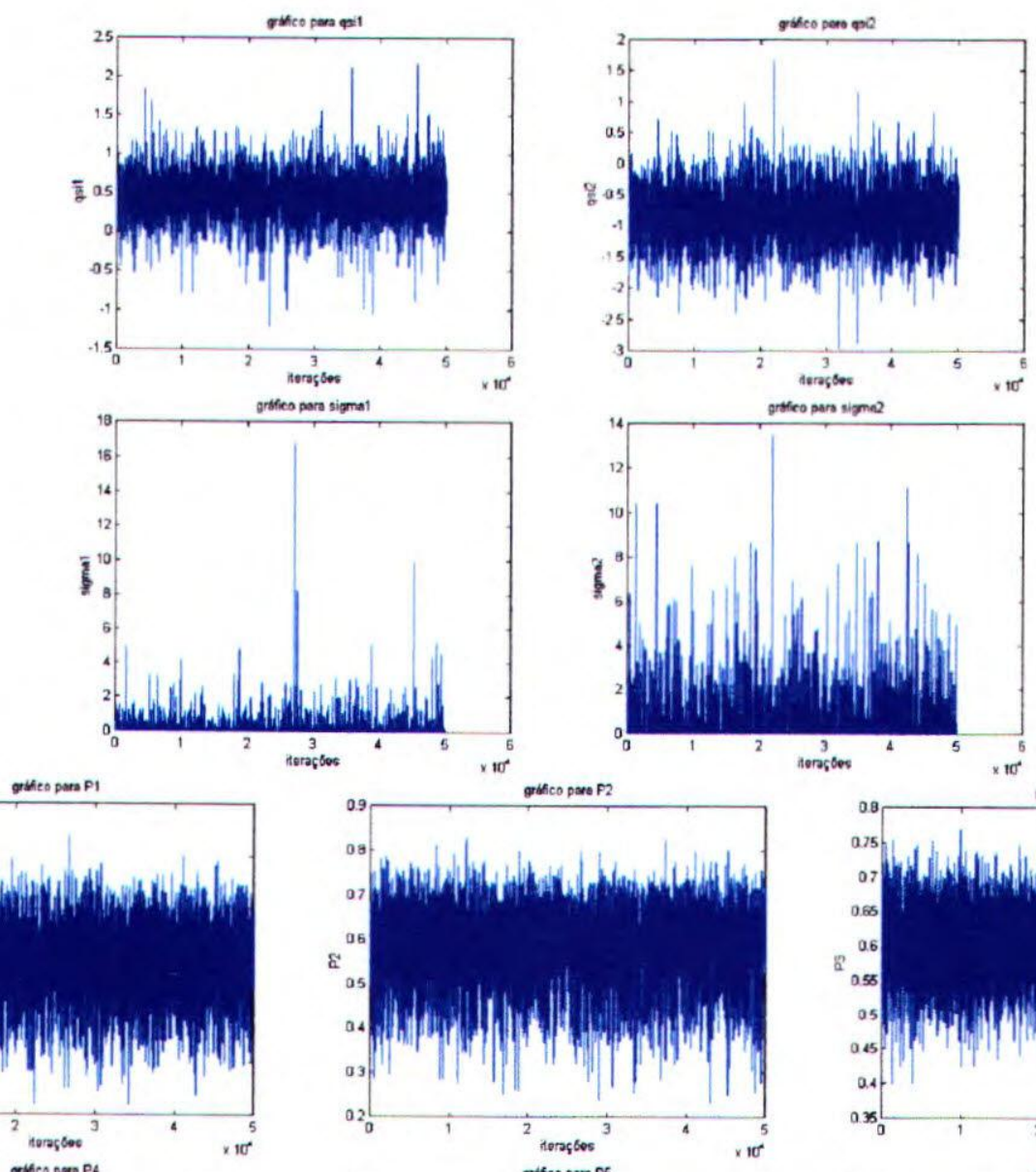

rthes perar sigmat
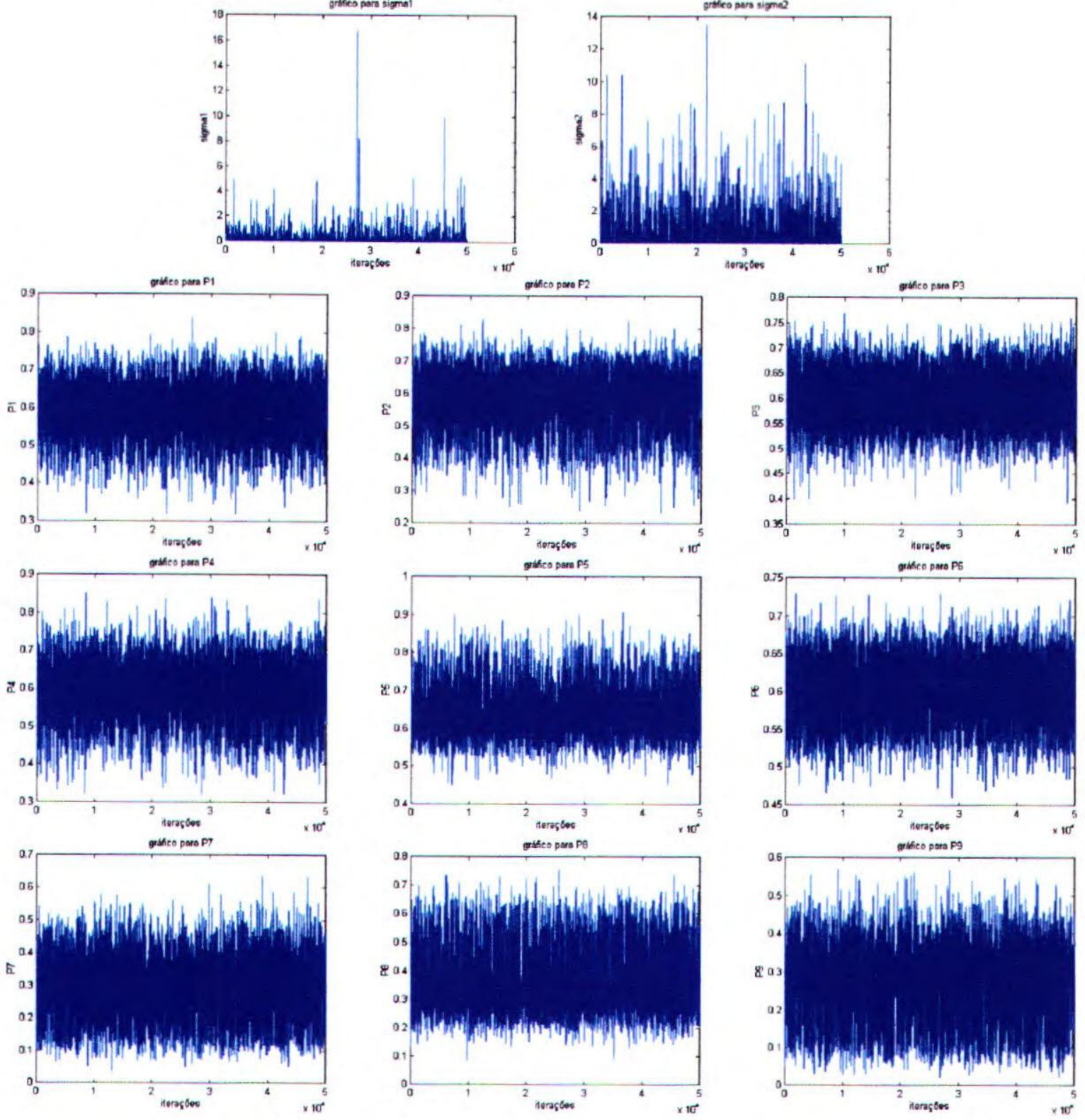

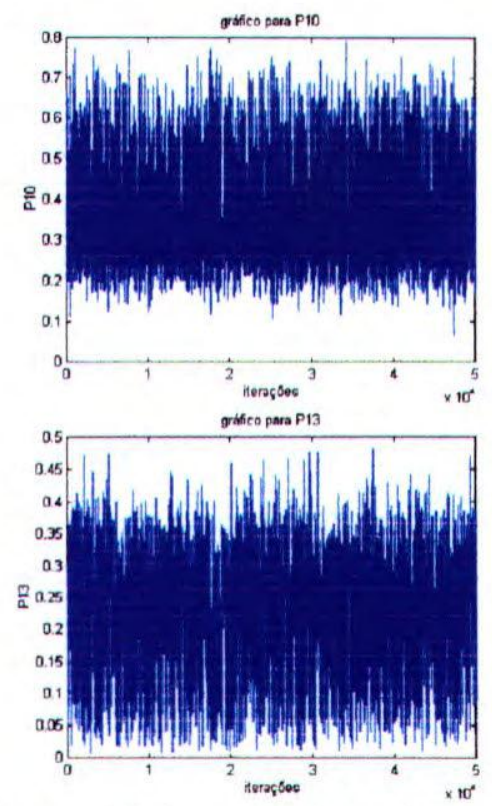

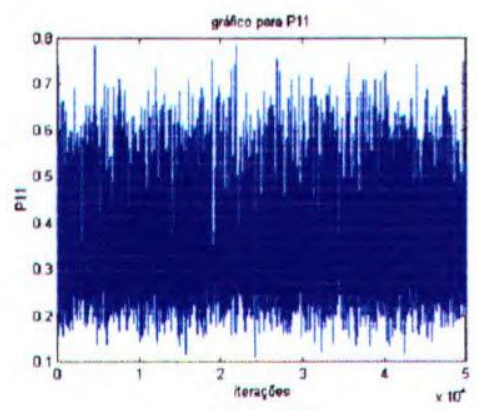

gratico para P14

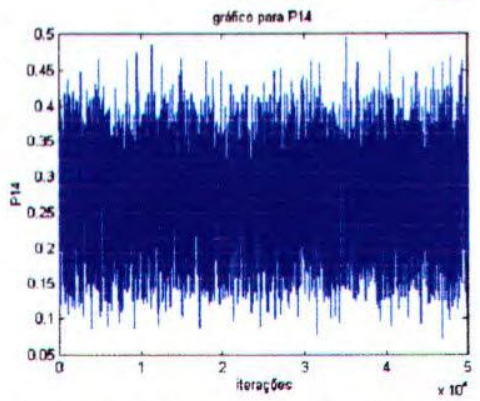

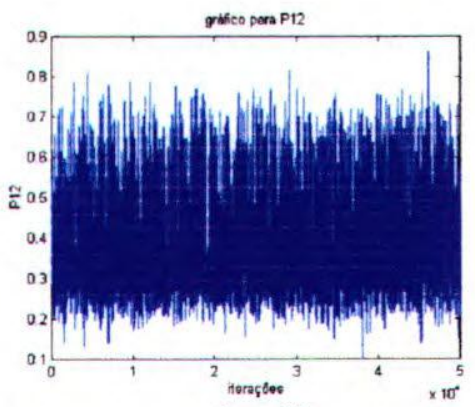

guco para P15

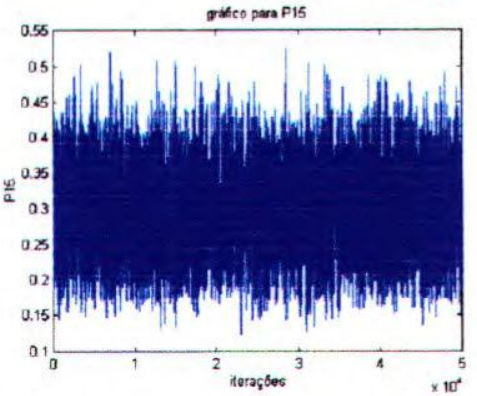

Figura 5: Convergência das amostras geradas para $\xi_{1}, \xi_{2}, \sigma_{1}^{2}, \sigma_{2}^{2}, p_{i}, i=1, \ldots, 15$, respectivamente.

Os gráficos de autocorrelação para cada um dos parâmetros, antes e depois de fazermos uma seleção da amostra gerada foram analisadas e estavam sob controle.

A parte computacional deste trabalho foi desenvolvida utilizando o software MATLAB, gerando cinco cadeias com 10000 valores cada, totalizando uma amostra de 50000 valores gerados. Desses 50000 valores, foi selecionada uma amostra de 3500 valores. Esses 3500 valores foram obtidos desprezando $30 \%$ dos valores iniciais gerados por cada cadeia e os $70 \%$ restantes foram selecionados de 10 em 10, pretendendo com isso, assegurar a suposição de independência.

A convergência dos parâmetros foi verificada utilizando o critério de convergência dado por Gelman e Rubin (1992) (ver Tabela 3). 
Tabela 3: Valores usados como indicador de convergência, obtidos aplicando o critério de convergência dado por Gelman e Rubin (1992)

\begin{tabular}{cc}
\hline Parâmetros & Valores Obtidos $(\sqrt{\hat{\boldsymbol{R}}})$ \\
\hline$\xi_{1}$ & 0.9999 \\
$\xi_{2}$ & 1.0018 \\
$\sigma_{1}^{2}$ & 1.0219 \\
$\sigma_{2}^{2}$ & 1.0045 \\
$p_{1}$ & 1.0003 \\
$p_{2}$ & 1.0003 \\
$p_{3}$ & 1.0005 \\
$p_{4}$ & 1.0008 \\
$p_{5}$ & 0.9998 \\
$p_{6}$ & 0.9988 \\
$p_{7}$ & 1.0002 \\
$p_{8}$ & 1.0012 \\
$p_{9}$ & 1.0003 \\
$p_{10}$ & 1.0023 \\
$p_{11}$ & 1.0028 \\
$p_{12}$ & 1.0013 \\
$p_{13}$ & 1.0014 \\
$p_{14}$ & 1.0012 \\
$p_{15}$ & 1.0028 \\
\hline
\end{tabular}

Analisando os resultados da Tabela 3, podemos concluir pela convergência dos parâmetros, já que os valores obtidos estão de acordo com o valor recomendado pelo critério de Gelman e Rubin que é aproximadamente 1.

\subsection{Verificação da adequação do modelo}

A adequabilidade do modelo foi verificada utilizando o procedimento proposto na Seção 2.6 com a estatística de teste definida em (2.10). Desta forma, o valor do nível descritivo $\left(p_{v}\right)$ definido em (2.11) foi de 0.7829. Como este valor está dentro do intervalo de aceitação, ou seja, $0.05<\hat{p}_{v}<0.95$, podemos concluir que o modelo está adequado aos dados. 


\section{CAPÍTULO 4 - UM PROCEDIMENTO CLÁSSICO EM META-ANÁLISE}

\subsection{Introdução}

A literatura estatística apresenta diversas formas para combinar informações de vários estudos independentes, em geral com uma grande massa de dados. Essas técnicas estão sendo propostas desde Tippett (1931). Uma excelente revisão das técnicas clássicas, em geral, não paramétricas combinando vários p-valores e outros procedimentos é dada por Hedges (1992). Podemos destacar nesta direção os trabalhos de Tippett (1931), Fisher (1932), Stouffer, Suchman, De Vinney, Star e Willians (1949), e George (1977).

Neste capítulo, em particular, vamos apresentar uma análise clássica para o exemplo de aplicação desenvolvido no capítulo 3 , baseada em testes de hipóteses e intervalos de confiança. Esta análise é desenvolvida simplesmente para termos uma noção dos resultados obtidos por uma meta-análise clássica em comparação a meta-análise Bayesiana. Para isso, vamos fazer uma comparação entre os intervalos de confiança (análise clássica) e intervalos de credibilidade (análise Bayesiana) obtidos no capítulo 3.

\subsection{Comparação de proporções de várias amostras independentes}

Consideremos que $y$ é a freqüência com que foi observada alguma característica da amostra. A freqüência $y$ é uma estatística, pois é determinada em função dos elementos da amostra. Evidentemente, podemos, para cada elemento da amostra, considerar a ocorrência de um sucesso, caso a característica desejada se verifique, e de um fracasso, caso contrário. Desta 
forma, temos que $y$ segue uma distribuição Binomial com parâmetros $n$ e $p$, seguindo-se, pelas propriedades da distribuição Binomial,

$$
E(y)=n p \text { e } \operatorname{Var}(y)=n p(l-p)
$$

Seja $p$ a probabilidade de ocorrência de sucesso para cada elemento da amostra. A freqüência relativa $\hat{p}$, por sua vez, sendo simplesmente o quociente de $y$ pelo tamanho da amostra $n$, tem sua esperança e variância dadas, respectivamente, por:

$$
E(\hat{p})=E\left(\frac{y}{n}\right)=\frac{l}{n} n p=p \text { e } \operatorname{Var}(\hat{p})=\operatorname{Var}\left(\frac{y}{n}\right)=\frac{l}{n^{2}} n p(l-p)=\frac{p(l-p)}{n}
$$

Os possiveis valores de $\hat{p}$, se encontram entre 0 e 1 com intervalos de comprimento $\frac{1}{n}$.

Sendo a amostra suficientemente grande, podemos aproximar as distribuiçōes de $y$ e $\hat{p}$ por distribuiçōes Normais utilizando o Teorema do Limite Central. Em termos práticos, em geral, podemos considerar que a amostra será suficientemente grande, para efeito dessa aproximação, se $n p \geq 5$ e $n(l-p) \geq 5$ ( no caso de não conhecermos a proporção populacional, adotaremos como condições de aproximação $n \hat{p} \geq 5$ e $n(l-\hat{p}) \geq 5$ ).

Para este estudo, onde temos 15 amostras independentes e já que $n_{i} \hat{p}_{i} \geq 5$ e $n_{i}\left(1-\hat{p}_{i}\right) \geq 5$ para todo $i, i=1, \ldots, 15$ (exceto para $i=9$ e $i=13$ ), vamos aproximar as distribuições de $y_{i}$ e $\hat{p}_{i}$ por distribuições Normais de mesma média e mesmo desvio-padrão.

Se cada $\hat{p}_{i}, i=1, \ldots, 15$, são valores aleatórios de variáveis independentes retirados de uma população Normal de média $n p$ e desvio-padrão $\sqrt{n p(l-p)}$, diremos que a estatística

$$
\chi_{v}^{2}=\sum_{i=1}^{v}\left(\frac{\hat{p}_{i}-n p}{\sqrt{n p(l-p)}}\right)^{2}=\sum_{i=1}^{v} Z_{i}^{2}
$$


tem distribuição $\chi^{2} \operatorname{com} v$ graus de liberdade.

Os valores de $Z_{I}$ na expressão acima, são os correspondentes valores da variável Normal-padrão. Podemos, portanto, considerar a distribuição da variável $\chi^{2} \operatorname{com} \nu$ graus de liberdade como a soma dos quadrados de $v$ valores independentes da variável Normal-padrão. Temos ainda que

$$
E\left(\chi_{v}^{2}\right)=v \text { e } \operatorname{Var}\left(\chi_{v}^{2}\right)=2 v
$$

Outra importante propriedade das distribuições tipo $\chi^{2}$ é sua aditividade. Essa propriedade significa que a soma de duas variáveis independentes com distribuições $\chi^{2} \operatorname{com} v_{t}$ e $v_{2}$ graus de liberdade terá também distribuição $\chi^{2} \operatorname{com} v_{1}+v_{2}$ graus de liberdade.

Partindo destas definições, Fleiss (1973) propôs algumas técnicas para comparar $N$ proporções sumarizando os resultados de vários estudos independentes.

Em particular, vamos utilizar os dados da Tabela 1 (ver seção 3.1) para exemplificar o uso destas técnicas.

\subsubsection{Teste para verificar a igualdade de $N$ proporções}

Vamos designar por $H_{o}$ a hipótese existente, a ser testada, e por $H_{a}$ a hipótese alternativa. O teste irá levar à aceitação ou rejeição da hipótese $H_{0}$, o que corresponde, portanto, à negação ou afirmação de $H_{a}$.

Considere $N$ amostras independentes. Fleiss traz um teste para verificar quais amostras ou grupos de amostras que levam a uma diferença significativa. Para a realização dos testes, vamos definir os valores para as proporções referentes a cada estudo e a proporção média, descritos, respectivamente, por, 


$$
\hat{p}_{1}=\frac{y_{i}}{n_{i}}=\frac{n_{i t}}{n_{i .}} \quad \text { e } \quad \bar{p}=\frac{n_{\cdot i}}{n_{.}}=\frac{\sum n_{i \cdot} \hat{p}_{i}}{\sum n_{i .}}
$$

onde, Fleiss considera, $n_{i l}$ correspondendo ao número de pacientes que respondem ao tratamento no estudo $i, i=1, \ldots N ; n_{t}$ correspondendo ao número de pacientes que completaram o estudo $i, i=1,2, \ldots, N$ e $n_{.,}$o número total de pacientes, ou seja, $n=\sum_{i=1}^{N} n_{i}, i=1, \ldots, N$;

Portanto, para verificarmos a igualdade das $N$ proporções, devemos testar as hipóteses:

$$
\left\{\begin{array}{l}
H_{0}: p_{1}=p_{2}=\ldots=p_{N} \\
H_{a}: p_{i} \neq p_{j}
\end{array} \quad \text { para algum } i \neq j .\right.
$$

Para isso, utilizamos a estatística de teste dada por Fleiss (1973) definida por,

$\chi_{O B S}^{2}=\frac{l}{\bar{p} \bar{q}} \sum_{i=1}^{N} n_{i .}\left(\hat{p}_{i}-\bar{p}\right)^{2}$

onde $\bar{q}=1-\bar{p}$.

A estatística de teste (4.1) pode ser comparada com uma distribuição Qui-quadrado com $N-1$ graus de liberdade. Portanto, a hipótese de igualdade $H_{0}$ é rejeitada se $\chi_{O B S}^{2}>\chi_{N-1}^{2}$.

\subsubsection{Teste para verificar a igualdade entre dois grupos de estudo}

Agora, supondo $N$ amostras particionadas em dois grupos; o primeiro contendo $N_{1}$ amostras e segundo contendo $N_{2}$ amostras, sendo $N_{1}+N_{2}=N$, definimos:

$n_{(l)}=\sum_{i=1}^{N_{i}} n_{i .}$ como sendo o número total de observações no primeiro grupo de amostras,

$n_{(2)}=\sum_{i=N_{i}+1}^{N} n_{i .}$ como sendo o número total de observaç̃os no segundo grupo de amostras, 
$\bar{p}_{l}=\frac{\sum_{i=l}^{N_{t}} n_{i} \hat{p}_{t}}{n_{(1)}}$ a proporção média no primeiro grupo e,

$\bar{p}_{2}=\frac{\sum_{i=N_{1}+1}^{N} n_{i} \hat{p}_{i}}{n_{(2)}}$ como sendo a proporção média no segundo grupo.

A partir das definições acima, um teste para verificar se existe diferenças significativas entre as proporções dos dois grupos, considera as hipóteses abaixo,

$$
\left\{\begin{array}{l}
H_{0}: p_{N_{1}}=p_{N_{2}} \\
H_{a}: p_{N_{1}} \neq p_{N_{2}}
\end{array}\right.
$$

onde, a estatística para testar a hipótese acima é dada por (Fleiss, 1973),

$$
\chi_{(d f)}^{2}=\frac{l}{\bar{p} \bar{q}} \frac{n_{(1)} n_{(2)}}{n}\left(\bar{p}_{1}-\bar{p}_{2}\right)^{2}
$$

que pode ser comparada com uma distribuição Qui-quadrado com 1 grau de liberdade. Assim, rejeitamos a hipótese $H_{0}$ se $\chi_{(d f)}^{2}>\chi_{1}^{2}$.

\subsubsection{Teste para verificar a igualdade dentro de grupos}

Para verificar se existe diferenças significativas das $N_{1}$ proporçōes dentro do primeiro grupo, definimos as hipóteses:

$$
\left\{\begin{array}{l}
H_{0}: p_{1}=p_{2}=\ldots=p_{N_{1}} \\
H_{a}: p_{i} \neq p_{j}
\end{array} \quad \text { para algum } i \neq j\right.
$$

cuja estatística de teste é dada por, 


$$
\chi_{\text {gnipol }}^{2}=\frac{l}{\bar{p} \bar{q}} \sum_{i=l}^{N_{1}} n_{i .}\left(\hat{p}_{i}-\bar{p}_{l}\right)^{2}
$$

podendo ser comparada com uma distribuição Qui-quadrado com $N_{1}-l$ graus de liberdade, rejeitando a hipótese de igualdade das $N_{1}$ proporções se $\chi_{g \text { rupol }}^{2}>\chi_{N_{1}-1}^{2}$.

De forma análoga, para testar se há diferenças significativas das $N_{2}$ proporções dentro do segundo grupo, definimos as hipóteses:

$$
\left\{\begin{array}{l}
H_{0}: p_{N_{t}+1}=p_{N_{t}+2}=\ldots=p_{N} \\
H_{a}: p_{i} \neq p_{j}
\end{array} \quad \text { para algum } i \neq j\right.
$$

com estatística de teste dada por,

$$
\chi_{g \text { nupo } 2}^{2}=\frac{1}{\bar{p} \bar{q}} \sum_{t=N_{1}+1}^{N} n_{i}\left(\hat{p}_{i}-\bar{p}_{2}\right)^{2}
$$

a qual, comparada com uma distribuição Qui-quadrado com $N_{2}-l$ graus de liberdade, pode levar a rejeição da hipótese de igualdade das $N_{2}$ proporções se $\chi_{g r u p o 2}^{2}>\chi_{N_{2}-1}^{2}$.

\subsubsection{Teste para verificar se cada uma das $N$ proporçōes amostrais é igual a $\frac{1}{2}$}

Verificadas as hipóteses descritas nas seções 4.1.1, 4.1.2 e 4.1.3, suponha que seja de interesse verificarmos se a proporção no estudo $i$ é igual a $\frac{1}{2}$, e, posteriormente, construirmos intervalos com $95 \%$ de confiança para cada $p_{i}, i=1, \ldots, N$. Para isso assumir

$$
y_{i} \sim \operatorname{Binomial}\left(n_{i}, p_{i}\right) \text { e } \hat{p}_{i}=\frac{y_{i}}{n_{i}}, \quad i=1, \ldots, N
$$


Em geral, para inferir sobre os parâmetros de um determinado modelo, é usual utilizar a aproximação normal assintótica dos estimadores de máxima verossimilhança (ver por exemplo, Mood, Graybill e Boes, 1974). Então, consideramos

$$
y_{i} \sim \operatorname{Normal}\left(n_{i} p_{t}, n_{i} p_{l}\left(1-p_{i}\right)\right)
$$

As hipóteses a serem testadas para cada estudo $i, i=1, \ldots, N$ são definidas da seguinte forma:

$$
\left\{\begin{array}{l}
H_{o i}: p_{i}=\frac{1}{2} \\
H_{a i}: p_{i} \neq \frac{1}{2}
\end{array}\right. \text {. }
$$

A estatística de teste sob $H_{0}$ é dada por,

$$
Z_{i}=\frac{\hat{p}_{i}-\frac{1}{2}}{\sqrt{\frac{\hat{p}_{i}\left(1-\hat{p}_{i}\right)}{n_{i}}}} \sim \operatorname{Normal}(0,1) .
$$

Desta forma, a hipótese $H_{o i}$ definida acima será rejeitada quando $\left|Z_{i}\right| \geq 1.96$ para cada proporção $p_{i}, i=1, \ldots, N$.

\subsection{Intervalos com $95 \%$ de confiança para cada uma das $N$ proporções}

Um intervalo com $95 \%$ de confiança para cada $p_{i} i=1, \ldots, N$ será definido da seguinte forma:

Sejam,

$$
Z_{i}=\frac{y_{i}-n_{i} \hat{p}_{l}}{\sqrt{n_{i} \hat{p}_{i}\left(1-\hat{p}_{i}\right)}} \stackrel{\circ}{\sim} \operatorname{Normal}(0,1)
$$

e 


$$
\hat{p}_{i}=\frac{y_{i}}{n_{i}} \stackrel{a}{\sim} \operatorname{Normal}\left(p_{i}, \frac{p_{i}\left(1-p_{i}\right)}{n_{i}}\right) .
$$

então, $Z_{i}$ pode ser escrito como,

$$
Z_{i}=\frac{\hat{p}_{i}-p_{i}}{\sqrt{\frac{\hat{p}_{i}\left(l-\hat{p}_{i}\right)}{n_{i}}}} \sim \operatorname{Normal}(0, I) .
$$

Portanto, a probabilidade de $Z_{i}$ estar contido no intervalo $(-1.96 ; 1.96)$, com $95 \%$ de confiança pode ser expressa por,

$$
P\left\{-1.96 \leq \frac{\hat{p}_{1}-p_{i}}{\sqrt{\hat{p}_{1}\left(I-\hat{p}_{1}\right) / n_{1}}} \leq 1.96\right\}=0.95
$$

Assim, um intervalo com $95 \%$ de confiança para cada proporção $p_{i} i=1, \ldots . N$, é dado por:

$$
\left[\hat{p}_{i}-1.96 \sqrt{\frac{\hat{p}_{i}\left(I-\hat{p}_{i}\right)}{n_{i}}} ; \hat{p}_{i}+1.96 \sqrt{\frac{\hat{p}_{i}\left(I-\hat{p}_{i}\right)}{n_{i}}}\right]
$$

\subsection{Resultados}

Após a introdução, nas seções 4.1.1 à 4.1.4, de alguns procedimentos clássicos para testar hipóteses de interesse e considerando os dados da Tabela 1 (ver seção 3.1) como ilustração, temos os seguintes resultados considerando um nivel de significância $\alpha=5 \%$ :

Da expressão (4.1), temos, 


$$
\chi_{O B S}^{2}=\frac{1}{0.4797(0.5203)} \sum_{i=1}^{15} n_{i .}\left(\hat{p}_{i}-0.4797\right)^{2}=72.4144
$$

e

$$
\chi_{N-1}^{2}=\chi_{14}^{2}=23.685
$$

Como $\chi_{O B S}^{2}>\chi_{14}^{2}$, rejeitamos a hipótese de igualdade dos $p_{i}{ }^{\prime} s$ com $\alpha=5 \%$ de significância, ou seja, podemos dizer que existe diferenças significativas entre as proporçôes $p_{i}$, $i=1, \ldots, 15$.

A partir da expressão (4.2), podemos afirmar que existe uma diferença significativa entre as proporções médias dos dois grupos, pois:

$$
\chi_{\text {(df) }}^{2}=\frac{1}{0.4797(0.5213)} \frac{331(236)}{567}(0.6072-0.3008)^{2}=51.8321
$$

e

$$
\chi_{1}^{2}=3.841
$$

Com esta diferença a favor da estatística $x_{(\text {dif })}^{2}$ podemos concluir que a diferença entre os dois grupos é significativa.

Para as expressőes dadas em (4.3) e (4.4) temos, respectivamente,

$$
\chi_{\text {grupol }}^{2}=5.2472 \quad \text { e } \quad \chi_{N_{1}-1}^{2}=\chi_{s}^{2}=11.070
$$

e,

$$
\chi_{\text {gnupo } 2}^{2}=15.348 \quad \text { e } \quad \chi_{N_{1}-1}^{2}=\chi_{s}^{2}=15.507 .
$$

Com estes resultados, concluímos que não há diferenças significativas dentro dos grupos, pois $\chi_{\text {grupol }}^{2}<\chi_{s}^{2}$ e $\chi_{\text {grupos }}^{2}<\chi_{8}^{2}$.

Considerando ainda, um nivel $\alpha=5 \%$, os resultados dos testes da seção 4.1 .4 e os intervalos de confiança dados pela expressão (4.10), estão descritos na Tabela 4. 
Tabela 4: Resultados dos testes de hipóteses $\left(H_{0 i}: p_{i}=\frac{1}{2}\right)$ e dos intervalos com $95 \%$ de confiança para cada $p_{i} i=1, \ldots, 15$

\begin{tabular}{ccccc}
\hline Estudo & $\hat{\boldsymbol{p}}_{\boldsymbol{i}}$ & $\boldsymbol{Z}_{\boldsymbol{i}}$ & Conclusão dos testes & $\begin{array}{c}\text { Intervalos de } \\
\text { confiança (95\%) }\end{array}$ \\
\hline 1 & 0.5666 & 0.73613 & Não rejeita $H_{o 1}$ & $(0.3893 ; 0.7439)$ \\
2 & 0.5000 & 0.00000 & Não rejeita $H_{02}$ & $(0.2550 ; 0.7450)$ \\
3 & 0.5942 & 1.59350 & Não rejeita $H_{03}$ & $(0.4783 ; 0.7101)$ \\
4 & 0.5652 & 0.63076 & Não rejeita $H_{04}$ & $(0.3626 ; 0.7678)$ \\
5 & 0.7619 & 3.98503 & Rejeita $H_{o 5}$ & $(0.6331 ; 0.8907)$ \\
6 & 0.5960 & 2.40406 & Rejeita $H_{o 6}$ & $(0.5177 ; 0.6743)$ \\
7 & 0.2500 & -2.58199 & Rejeita $H_{o 7}$ & $(0.0602 ; 0.4398)$ \\
8 & 0.4500 & -0.44947 & Não rejeita $H_{08}$ & $(0.2320 ; 0.6680)$ \\
9 & 0.1765 & -3.49860 & Rejeita $H_{o 9}$ & $(-0.0047 ; 0.3577)$ \\
10 & 0.4667 & -0.25851 & Não rejeita $H_{o 10}$ & $(0.2142 ; 0.7192)$ \\
11 & 0.4444 & -0.47473 & Não rejeita $H_{o 11}$ & $(0.2148 ; 0.6740)$ \\
12 & 0.5294 & 0.24286 & Não rejeita $H_{o 12}$ & $(0.2921 ; 0.7667)$ \\
13 & 0.0526 & -8.73601 & Rejeita $H_{o 13}$ & $(-0.0478 ; 0.1530)$ \\
14 & 0.2353 & -4.45638 & Rejeita $H_{o 14}$ & $(0.1189 ; 0.3517)$ \\
15 & 0.2881 & -3.59398 & Rejeita $H_{o 15}$ & $(0.1725 ; 0.4037)$ \\
\hline
\end{tabular}

A Tabela 4 mostra, para cada estudo, a proporção estimada de pacientes melhorados $\left(\hat{p}_{i}\right)$, os valores da estatística de teste $Z_{i}$ para cada estudo, $i=1, \ldots, 15$, os resultados do teste dado na seção 4.1.4 para cada estudo e os intervalos de confiança para cada proporção $p_{i}$.

Analisando os resultados dos testes de hipóteses dados na seção 4.1.4, podemos dizer que os estudos $5,6,7,9,13,14$ e 15 são significativamente diferentes de $\frac{1}{2}$ quando comparados a uma distribuição Normal-padrão com um nível de significância $\alpha=5 \%$.

Uma conclusão geral sobre os resultados obtidos neste capítulo é que existe diferenças significativas entre as proporções e entre os grupos de estudo, mas uma série de questões ainda permanecem não esclarecidas, pois inferências baseadas somente em testes de hipóteses não fornecem informações a respeito do tamanho do efeito do tratamento. 
Neste sentido, a análise Bayesiana apresenta resultados mais conclusivos e quando comparadas em termo dos intervalos fornecidos, os intervalos de credibilidade para os $p_{i}$ 's, em geral, se apresentam mais "fechados" com relação aos intervalos de confiança. 


\section{CAPÍTULO 5 - UM EXEMPLO SIMULADO PARA ILUSTRAR A COMPARAÇÃO ENTRE DOIS MATERIAIS USADOS PARA FABRICAR SOLAS DE SAPATOS}

\subsection{Introdução}

É comum na prática a comparação de dois tratamentos, seja clínico, industrial ou qualquer que seja a área de interesse. Para ilustrarmos um caso comum, de interesse industrial, vamos utilizar um exemplo simulado onde uma indústria está interessada em saber se um novo material para fabricar solas de sapatos é superior a um tradicional.

O conjunto de dados introduzidos na Tabela 5 são resumos de um conjunto de dados gerados aleatoriamente utilizando o software MINITAB.

A variável resposta para este experimento foi definida como a diferença média na espessura das solas dos sapatos $\left(\bar{D}_{i}\right)$ entre crianças com uma certa faixa etária pertencentes à escolas localizadas em bairros de diferentes classes sociais. Cada uma das crianças usaram os dois tipos de solas, ou seja, um tipo no pé direito e outro no pé esquerdo distribuídas aleatoriamente. 
Tabela 5: Dados de 10 estudos independentes sobre a diferença (na espessura) entre dois materiais usados para fabricar solas de sapatos.

\begin{tabular}{cccccc}
\hline Localização & Estudo & Original & $\boldsymbol{n}_{\boldsymbol{i}}$ & $\overline{\boldsymbol{D}}_{\boldsymbol{i}}$ & $\boldsymbol{S}_{\boldsymbol{i}}$ \\
\hline & 1 & 2 & 23 & 0.201 & 0.487 \\
Bairros de classe & 2 & 5 & 67 & -0.4603 & 0.6039 \\
Média-alta & 3 & 6 & 22 & 0.0542 & 0.3548 \\
& & & & & \\
Bairros de classe & 4 & 1 & 54 & 0.8754 & 0.5644 \\
Pobre & 5 & 3 & 32 & 0.5594 & 0.2577 \\
& 7 & 4 & 101 & 0.4533 & 0.2372 \\
& 8 & 8 & 202 & 0.21089 & 0.832 \\
& 9 & 9 & 76 & 0.5558 & 0.2928 \\
& 10 & 10 & 92 & 0.4885 & 0.1851 \\
\hline
\end{tabular}

A Tabela 5 fornece: o número de cada estudo; o número de cada estudo no conjunto de dados originais; o total de crianças que participaram do estudo $i$; a diferença média entre o novo solado e o solado tradicional para cada estudo $\left(\bar{D}_{i}\right)$ e o desvio padrão amostral $\left(S_{i}\right)$ para o estudo $i, i=1, \ldots, 10$.

Neste exemplo simulado, queremos ilustrar um problema típico em indústrias de calçados, onde o interesse principal é verificar se um novo material para fabricar solas de sapatos é superior a um tradicionalmente usado. Para isso, definimos $x$ como sendo a espessura da sola com o novo material após dois meses de uso diário por crianças em escolas públicas, $z$ a espessura da sola com o material antigo após dois meses de uso diário por crianças em escolas públicas, $\left(x_{i}, z_{i}\right)$ são os pares de medidas para cada indivíduo, sendo uma em cada pé (esquerdo ou direito) $i=1, \ldots, I$ e $D_{i}=x_{i}-z_{i}, i=1, \ldots, I$.

Observar que $D_{i}>0$, o novo tratamento é superior, pois a espessura da sola é maior após dois meses de uso contínuo.

Para ilustrar melhor o problema, supomos que a indústria aplica este estudo em 10 grupos distintos de crianças entre 10 e 14 anos de idade em 10 colégios: 3 escolas situadas em bairros de classe média-alta e 7 situadas em bairros de classe baixa. 
Alguns objetivos da pesquisa relacionado com o interesse da indústria são:

- Verificar se o novo material leva a maior durabilidade dos sapatos.

- Verificar se o status social leva a resultados diferentes.

- Identificar possiveis grupos com resultados diferentes.

Para analisar os dados da Tabela 5, vamos utilizar modelos Bayesianos hierárquicos e uma suposição importante a ser considerada é que os estudos são uma amostra aleatória da população de interesse.

Redefinindo a notação dada anteriormente para facilitar a interpretação, vamos assumir:

$$
\begin{aligned}
& y_{i}=\bar{d}_{i}=\frac{1}{n_{i}} \sum_{j=1}^{n_{1}}\left(x_{i j}-z_{i j}\right) \quad i=1, \ldots, 10 \\
& d_{i j}=x_{i j}-z_{i j} \\
& S_{i}=\frac{1}{n_{i}-1} \sum_{j=1}^{n_{1}}\left(d_{i j}-\bar{d}_{i}\right)^{2} \quad i=1, \ldots, 10, \text { onde } S_{i} \text { é o desvio padrão amostral para o estudo } i .
\end{aligned}
$$

\subsection{Definição do modelo 1 e suas respectivas variáveis}

Um primeiro modelo hierárquico para analisar os dados da Tabela 5 é dado como segue:

Assumindo $\sigma_{i}^{2}$ conhecido, para simplificação, temos que,

$$
\begin{aligned}
& y_{i} \mid \alpha_{i}, \sigma_{i}^{2} \stackrel{\text { ind }}{\sim} \operatorname{Normal}\left(\alpha_{i}, \sigma_{i}^{2}\right), \quad i=1, \ldots, I \\
& \alpha_{i} \mid \mu, \sigma_{\alpha}^{2} \stackrel{\text { iid }}{\sim} \operatorname{Normal}\left(\mu, \sigma_{a}^{2}\right), \quad i=1, \ldots, I
\end{aligned}
$$

onde: $y_{i}$ é o efeito do estudo observado, $i=1, \ldots, I$ $\alpha_{i}$ é o efeito do estudo verdadeiro, $i=1, \ldots, I$ 
$\dot{\sigma}_{i}^{2}$ é a variância dentro do estudo $i, i=I, \ldots, I$

$\mu$ é o efeito médio de estudo, $-\infty<\mu<\infty$, e

$\sigma_{\alpha}^{2}$ é a variância entre estudos, onde $\sigma_{\alpha}^{2}>0$

\subsubsection{Função de verossimilhança}

A partir do modelo especificado acima, definimos a função de verossimilhança por,

$$
\begin{gathered}
L\left(\alpha_{1}, \alpha_{2}, \ldots, \alpha_{1}\right)=\prod_{i=1}^{1} \frac{I}{\sqrt{2 \pi} \sigma_{i}} \exp \left\{-\frac{1}{2 \sigma_{i}^{2}}\left(y_{i}-\alpha_{i}\right)^{2}\right\} \\
\propto \exp \left\{-\frac{1}{2 \sigma_{i}^{2}} \sum_{i=1}^{I}\left(y_{i}-\alpha_{i}\right)^{2}\right\}
\end{gathered}
$$

\subsubsection{Distribuição a priori}

O modelo sugerido é definido com as distribuições a priori $\mu$ e $\sigma_{\alpha}^{2}$ especificadas abaixo:

$$
\begin{array}{lc}
\pi(\mu) \sim \operatorname{Normal}(a, b), \quad \mapsto(0,1) & -\infty<\mu<\infty \\
\pi\left(\sigma_{a}^{2}\right) \sim \text { Gama Inversa }(c, d), & \sigma_{a}^{2}>0
\end{array}
$$

onde, para o conjunto de dados da Tabela 5 , consideramos $a=0, b=1, c=2.9$ e $d=0.7$, onde $\underline{c}$ e $\underline{d}$ foram estimados através da média e variância de uma distribuição Gama Inversa.

Assumindo independência a priori, a distribuição a priori conjunta para $\alpha_{1}, \ldots, \alpha_{1}, \mu$ e $\sigma_{\alpha}^{2}$ é dada por:

$$
\begin{aligned}
\pi\left(\alpha_{1}, \ldots, \alpha_{1}, \mu, \sigma_{\alpha}^{2}\right) & \propto \sigma_{\alpha}^{-1} \exp \left\{-\frac{1}{2 \sigma_{\alpha}^{2}} \sum_{i=i}^{1}\left(\alpha_{i}-\mu\right)^{2}\right\} \times \exp \left\{-\frac{1}{2 b}(\mu-a)^{2}\right\} \\
& \times\left(\sigma_{a}^{2}\right)^{(c+1)} \exp \left\{-\frac{d}{\sigma_{a}^{2}}\right\}
\end{aligned}
$$




\subsubsection{Distribuição a posteriori conjunta}

Combinando (5.4) com (5.6), a distribuição a posteriori conjunta é dada por,

$$
\begin{aligned}
\pi\left(\alpha_{1}, \ldots, \alpha_{I}, \mu, \sigma_{\alpha}^{2} \mid D\right) \propto & \exp \left\{-\frac{1}{2 \sigma_{i}^{2}} \sum_{i=1}^{l}\left(y_{i}-\alpha_{i}\right)^{2}\right\} \times \sigma_{\alpha}^{-1} \exp \left\{-\frac{1}{2 \sigma_{\alpha}^{2}} \sum_{i=i}^{l}\left(\alpha_{i}-\mu\right)^{2}\right\} \times \\
& \times \exp \left\{-\frac{1}{2 b}(\mu-a)^{2}\right\} \times\left(\sigma_{\alpha}^{2}\right)^{-(c+1)} \exp \left\{-\frac{d}{\sigma_{\alpha}^{2}}\right\}
\end{aligned}
$$

A partir da distribuição a posteriori conjunta podemos alcançar os objetivos propostos na Seção 5.1, verificando o que é de interesse do pesquisador.

\subsubsection{Aspectos computacionais}

A partir da distribuição a posteriori conjunta, foi possivel encontrar as distribuições condicionais para $\alpha_{l}, \ldots, \alpha_{l}, \mu \mathrm{e} \sigma_{a}^{2}$,

$$
\begin{aligned}
& \pi\left(\alpha_{i} \mid \mu, \sigma_{\alpha}^{2}, \alpha_{\{i}, D\right) \propto N o r m a l\left[\mu\left(\frac{\sigma_{i}^{2}}{\sigma_{\alpha}^{2}+\sigma_{i}^{2}}\right)+y_{i}\left(\frac{\sigma_{\alpha}^{2}}{\sigma_{\alpha}^{2}+\sigma_{i}^{2}}\right), \frac{\sigma_{\alpha}^{2} \sigma_{i}^{2}}{\sigma_{\alpha}^{2}+\sigma_{i}^{2}}\right] \\
& \pi\left(\mu \mid \alpha_{i}, \ldots, \alpha_{1}, \sigma_{\alpha}^{2}, D\right) \propto N o r m a l\left[\frac{b I \bar{\alpha}+\sigma_{\alpha}^{2} a}{b I+\sigma_{\alpha}^{2}}, \frac{\sigma_{\alpha}^{2} b}{b I+\sigma_{\alpha}^{2}}\right]
\end{aligned}
$$

$\pi\left(\sigma_{\alpha}^{2} \mid \mu, \alpha_{1}, \ldots, \alpha_{I}, D\right) \propto$ Gama Inversa $\left[\frac{I}{2}+c, \frac{\sum_{i=1}^{I}\left(\alpha_{i}-\mu\right)^{2}+2 d}{2}\right]$

onde $\bar{\alpha}=\frac{\sum_{i=I}^{I} \alpha_{I}}{I}$ e $\alpha_{(i)}=\left(\alpha_{1}, \ldots, \alpha_{i-l}, \alpha_{i+l}, \ldots, \alpha_{l}\right)$

Com o uso do algoritmo Gibbs Sampling foi possível gerat amostras das distribuições condicionais definidas em (5.8), (5.9) e (5.10). 


\subsubsection{Resultados}

Utilizando o modelo 1 definido na Seção 5.2, obtemos os resultados que estão descritos na Tabela 6.

Tabela 6: Médias a posteriori e intervalos com $95 \%$ de credibilidade para cada um dos parâmetros.

\begin{tabular}{ccc}
\hline Parâmetros & Médias a posteriori & Intervalos de credibilidade (95\%) \\
\hline$\mu$ & 0.3478 & $(-0.2452 ; 0.9573)$ \\
$\sigma_{\alpha}^{2}$ & 0.8599 & $(0.2780 ; 2.3061)$ \\
$\alpha_{1}$ & 0.2481 & $(-0.6021 ; 1.1585)$ \\
$\alpha_{2}$ & -0.1895 & $(-1.2476 ; 0.8036)$ \\
$\alpha_{3}$ & 0.1033 & $(-0.5291 ; 0.7776)$ \\
$\alpha_{4}$ & 0.7215 & $(-0.2658 ; 1.7352)$ \\
$\alpha_{5}$ & 0.5317 & $(0.0341 ; 1.0041)$ \\
$\alpha_{6}$ & 0.4324 & $(-0.0242 ; 0.8895)$ \\
$\alpha_{7}$ & 0.6020 & $(-0.5364 ; 1.8730)$ \\
$\alpha_{8}$ & 0.2161 & $(0.0178 ; 0.4093)$ \\
$\alpha_{9}$ & 0.5259 & $(-0.0338 ; 1.0381)$ \\
$\alpha_{10}$ & 0.4751 & $(0.1564 ; 0.8352)$ \\
\hline
\end{tabular}

Analisando a Tabela 6 , notamos que $\mu>0$, ou seja, quando todos os estudos são analisados conjuntamente, o efeito médio do estudo indica que o novo material utilizado para fabricar solas de sapatos leva a uma durabilidade maior, mostrando um desgaste de aproximadamente $0.35 \mathrm{~cm}$ menor que o tradicional após dois meses de uso diário. Apesar de ser positivo, o efeito $\mu$ não foi encontrado como sendo significativo. Portanto, uma afirmação positiva em relação ao novo material ser superior ao tradicional não pode ser dada.

A outra questão de interesse é saber se o status social leva a resultados diferentes. Para isso, analisamos as médias a posteriori para $\alpha_{i}, i=1, \ldots, 10$, e percebemos uma possível divisão de grupos, já que as médias a posteriori para $\alpha_{1}, \alpha_{2}$ e $\alpha_{3}$, que se referem ao grupo de escolas que estão localizadas em bairros de classe média-alta, se apresentam menores quando comparadas com o restante $\left(\alpha_{4}, \alpha_{5}, \alpha_{6}, \alpha_{7}, \alpha_{8}, \alpha_{9}\right.$ e $\left.\alpha_{10}\right)$, que se referem ao grupo de escolas que estão localizadas em bairros de baixo poder aquisitivo. Além disso, $\alpha_{5}, \alpha_{8}$ e $\alpha_{10}$, que pertencem ao 
segundo grupo, têm suas médias a posteriori positivas e significativamente diferentes de zero, como indica os intervalos de credibilidade para cada um deles.

\subsubsection{Análise gráfica}

A Figura 6 mostra a forma da distribuição marginal para cada um dos parâmetros
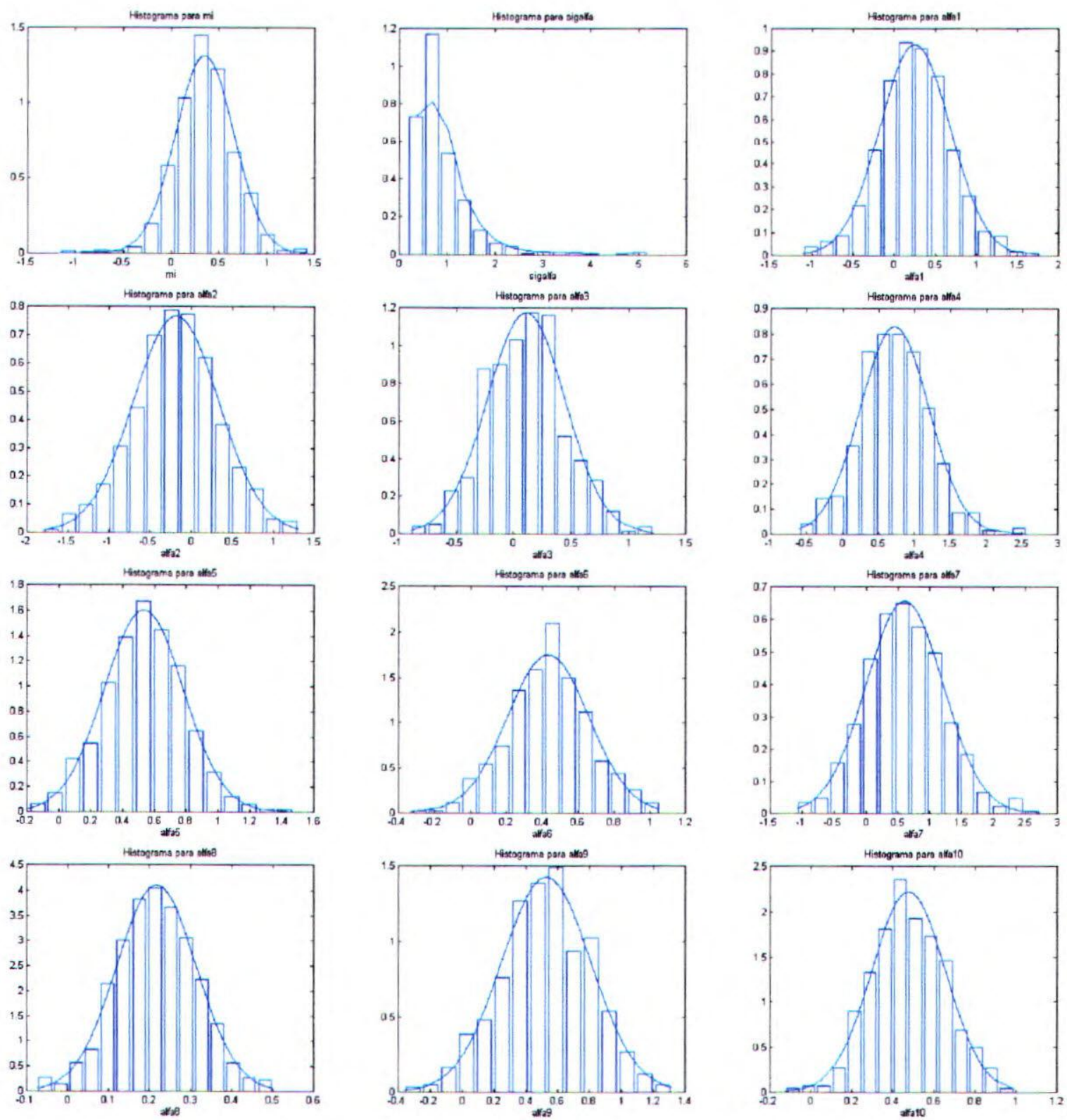

Figuras 5: Distribuições marginais a posteriori para $\alpha_{1}, \ldots, \alpha_{I}, \mu e \sigma_{\alpha}^{2}$, respectivamente. 
Na Figura 6 sobrepomos as formas das distribuições marginais para, podemos tirar conclusões graficamente analisando o deslocamento das médias $\alpha_{1}, \ldots, \alpha_{I}$ com o objetivo de possibilitar uma conclusão gráfica com respeito a um possível agrupamento das classes sociais.

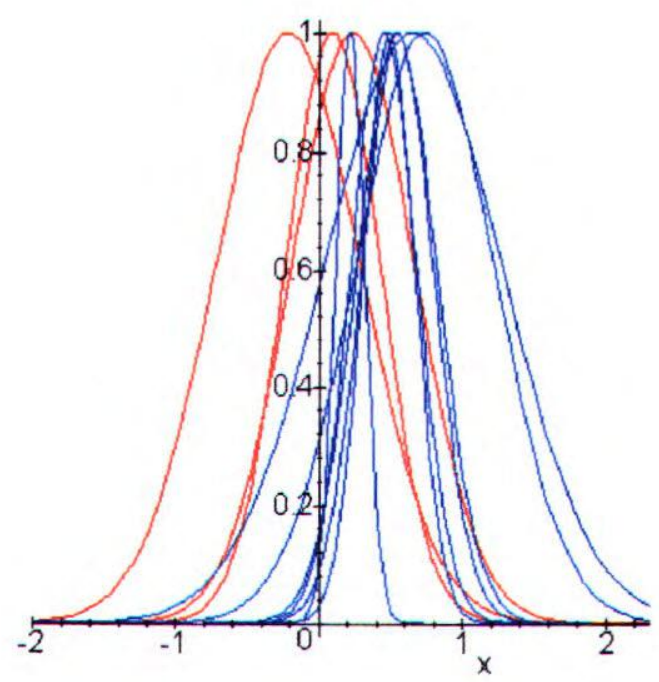

Figura 6: : Densidades a posteriori para $\alpha_{i}, i=1, \ldots, 10$ (classe média-alta: linhas vermelhas e classe pobre: linhas azuis).

Analisando a Figura 6, podemos observar um leve agrupamento da classe média-alta de um lado e classe pobre do outro, sugerindo uma possível diferença entre entre essas duas classes.

A Figura 7 mostra graficamente a convergência dos parâmetros, que foi confirmada através do critério de convergência apresentados por Gelman e Rubin (1992). 

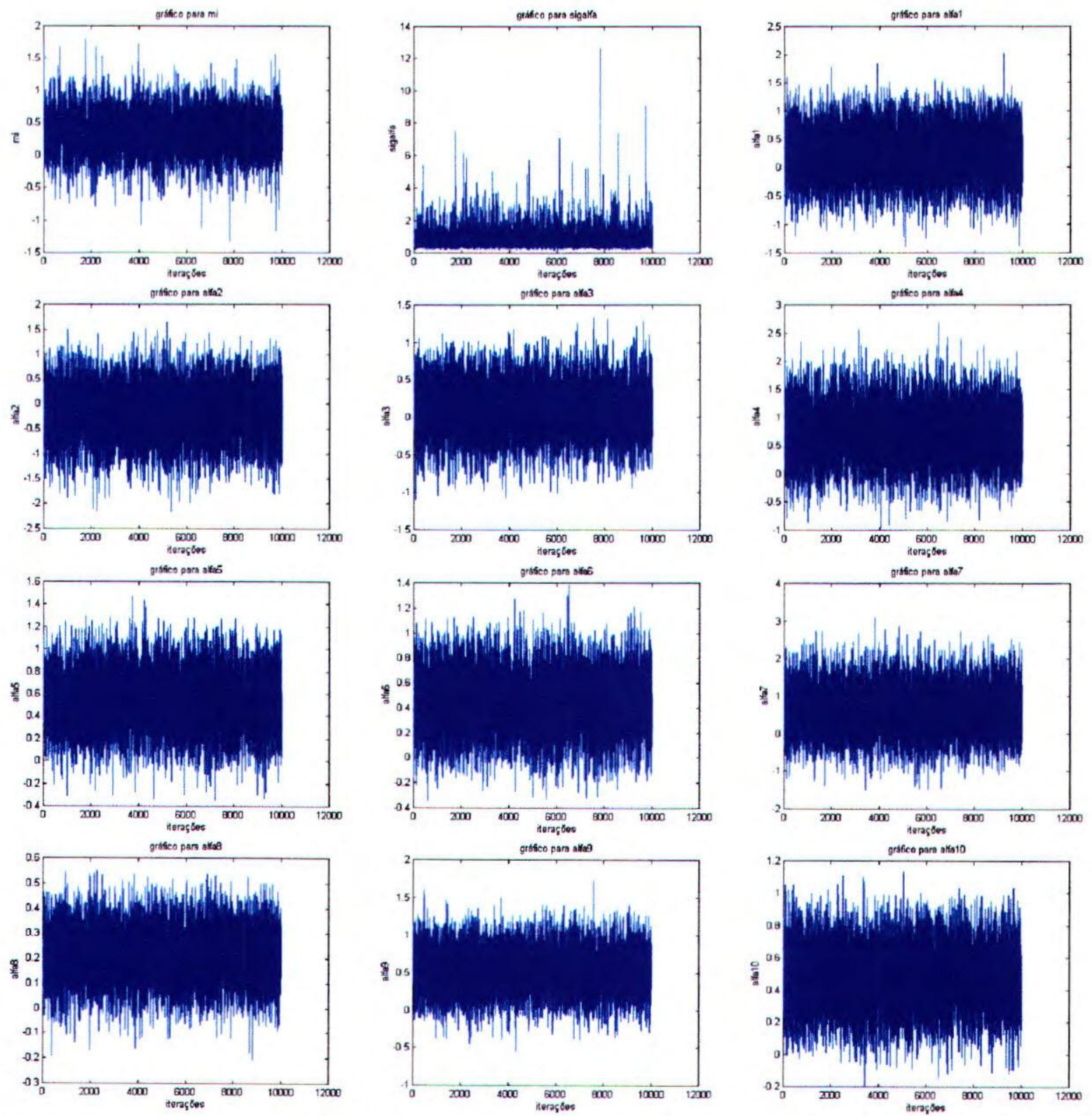

Figura 7: Gráficos com todos os valores gerados para $\alpha_{1}, \ldots, \alpha_{I}, \mu e \sigma_{\alpha}^{2}$ respectivamente.

Para este exemplo, a parte computacional também foi desenvolvida utilizando o software MATLAB, gerando cinco cadeias com 2000 valores cada, totalizando uma amostra de 10000 valores gerados. Desses 10000 valores, foi selecionada uma amostra de 600 valores. Esses 600 valores foram obtidos desprezando $40 \%$ dos valores iniciais gerados por cada cadeia e os $60 \%$ restantes foram selecionados de 10 em 10 .

Utilizando o critério de convergência dado por Gelman e Rubin (1992), os seguintes valores foram anotados: 
Tabela 7: Valores usados como indicador de convergência, obtidos aplicando o critério de convergência dado por Gelman e Rubin (1992)

\begin{tabular}{cc}
\hline Parâmetros & Valores Obtidos $(\sqrt{\hat{\boldsymbol{R}}})$ \\
\hline$\mu$ & 1.0020 \\
$\sigma_{\alpha}^{2}$ & 1.0059 \\
$\alpha_{1}$ & 1.0048 \\
$\alpha_{2}$ & 1.0114 \\
$\alpha_{3}$ & 1.0020 \\
$\alpha_{4}$ & 1.0036 \\
$\alpha_{5}$ & 1.0039 \\
$\alpha_{6}$ & 0.9928 \\
$\alpha_{7}$ & 0.9984 \\
$\alpha_{8}$ & 1.0273 \\
$\alpha_{9}$ & 1.0011 \\
$\alpha_{10}$ & 0.9985 \\
\hline
\end{tabular}

Analisando os resultados da Tabela 7, a convergência dos parâmetros pode ser confirmada através do critério proposto por Gelman e Rubin, visto que os valores obtidos estão próximos de 1 .

O segundo modelo hierárquico estudado com o intuito de obter resultados ainda mais claros é apresentado a seguir.

\subsection{Definição do modelo 2 e suas respectivas variáveis}

Para verificar se existe uma possível diferença entre grupos de estudo, consideramos um segundo modelo hierárquico para analisar os dados da Tabela 5. Este modelo considera um efeito aleatório para o grupo $k, k=1,2$. Desta forma podemos defini-lo como:

$$
\begin{aligned}
& y_{i} \mid \alpha_{i}, S_{i}^{2} \sim \operatorname{Normal}\left(\alpha_{i}, S_{i}^{2}\right), \quad i=1, \ldots, I \\
& \alpha_{i} \mid \mu_{i}, \delta_{k}, \sigma_{k}^{2} \sim \operatorname{Normal}\left(\mu_{i}+\delta_{k}, \sigma_{k}^{2}\right) \quad i=1, \ldots, I, k=1,2
\end{aligned}
$$


onde: $y_{i}$ é o efeito do estudo observado, $t=1, \ldots, I$

$\alpha_{i}$ é o efeito do estudo verdadeiro, $i=1, \ldots, I$

$S_{i}^{2}$ é a variância amostral dentro do estudo $i, i=1, \ldots, I$

$\mu_{i}$ é o efeito médio de estudo $i,-\infty<\mu<\infty, i=1, \ldots, I$

$\delta_{k}$ é o efeito aleatório para o grupo $k, k=1,2$

$\sigma_{k}^{2}$ é a variância para o grupo $k$, onde $\sigma_{k}^{2}>0, k=1,2$

Estamos considerando dois grupos de estudos: o grupo 1, formado pelas escolas localizadas em bairros de classe média -alta, contendo $I_{l}$ estudos, e o grupo 2 formado pelas escolas localizadas em bairros de classe pobre, contendo $I_{2}$ estudos. Portanto, $k=1$ representa $o$ grupo $1, k=2$ representa o grupo $2 \mathrm{e}, \mathrm{I}=I_{1}+I_{2}=10$.

\subsubsection{Função de verossimilhança}

Para este modelo, a função de verossimilhança é definida por:

$$
\begin{aligned}
L\left(\alpha_{1}, \ldots, \alpha_{l}\right)=\prod_{i=1}^{1} & \frac{1}{\sqrt{2 \pi} S_{i}} \exp \left\{-\frac{1}{2 S_{i}}\left(y_{i}-\alpha_{i}\right)^{2}\right\} \\
& \propto \exp \left\{-\frac{1}{2 S_{i}} \sum_{i=1}^{I}\left(y_{i}-\alpha_{i}\right)^{2}\right\}
\end{aligned}
$$

\subsubsection{Distribuição a priori}

O modelo sugerido é definido com as distribuições a priori especificadas a seguir:

$$
\begin{array}{ll}
\pi\left(\mu_{1}, \ldots, \mu_{I}\right) \propto \text { constante } & -\infty<\mu_{i}<\infty \\
\pi\left(\delta_{k}\right) \sim \operatorname{Normal}\left(a_{k}, b_{k}\right) & -\infty<\delta_{k}<\infty, k=1,2 \\
\pi\left(\sigma_{k}^{2}\right) \sim \text { Gama Inversa }\left(c_{k}, d_{k}\right) & \sigma_{k}^{2}>0, k=1,2
\end{array}
$$


onde, para o conjunto de dados da Tabela 5, consideramos $a_{k}=0, b_{k}=1, c_{k}=2.9$ e $d_{k}=0.7$.

Assumindo independência a priori, a distribuição a priori conjunta para $\alpha_{1}, \ldots, \alpha_{1}, \mu_{1}, \ldots, \mu_{1}, \delta_{1}, \delta_{2}, \sigma_{1}^{2}, \sigma_{2}^{2}$ é dada por:

$$
\begin{aligned}
\pi\left(\underset{\sim}{\mu} \underset{\sim}{\alpha}, \delta_{1}, \delta_{2}, \sigma_{1}^{2}, \sigma_{2}^{2}\right) \propto & \sigma_{1}^{-I_{1}-c_{1}-1} \sigma_{2}^{-I_{2}-c_{2}-1} \exp \left\{-\frac{1}{2 b_{1}}\left(\delta_{1}-a_{1}\right)^{2}-\frac{1}{2 b_{2}}\left(\delta_{2}-a_{2}\right)^{2}-\frac{d_{1}}{\sigma_{1}^{2}}-\frac{d_{2}}{\sigma_{2}^{2}}\right\} \times \\
& \times \exp \left\{-\frac{1}{2 \sigma_{1}^{2}} \sum_{i=1}^{I_{1}}\left(\alpha_{i}-\mu_{i}-\delta_{1}\right)^{2}-\frac{1}{2 \sigma_{2}^{2}} \sum_{i=I_{1}+1}^{l_{2}}\left(\alpha_{i}-\mu_{i}-\delta_{2}\right)^{2}\right\}
\end{aligned}
$$

\subsubsection{Distribuição a posteriori conjunta}

A distribuição a posteriori conjunta, obtida a partir de (5.14) e (5.15) é dada por:

$$
\begin{aligned}
\pi\left(\underset{\sim}{\mu, \alpha}, \delta_{1}, \delta_{2}, \sigma_{1}^{2}, \sigma_{2}^{2} \mid D\right) \propto \prod_{i=1}^{I} & \left.\left.\exp \left\{-\frac{1}{2 S_{1}^{2}}\left(y_{i}-\alpha_{i}\right)^{2}\right\}\left(\sigma_{1}^{2}\right)^{-\left(\frac{I_{1}}{2}+c_{1}+1\right.}\right)\left(\sigma_{2}^{2}\right)^{-\left(\frac{I_{2}}{2}+c_{2}+1\right.}\right) \times \\
& \times \exp \left\{-\frac{d_{1}}{\sigma_{1}^{2}}-\frac{d_{2}}{\sigma_{2}^{2}}-\frac{1}{2 b_{1}}\left(\delta_{i}-a_{1}\right)^{2}-\frac{1}{2 b_{2}}\left(\delta_{2}-a_{2}\right)^{2}\right\} \times \\
& \times \exp \left\{-\frac{1}{2 \sigma_{1}^{2}} \sum_{i=1}^{I_{1}}\left(\alpha_{i}-\mu_{i}-\delta_{1}\right)^{2}-\frac{1}{2 \sigma_{2}^{2}} \sum_{i=I_{j}+1}^{I_{2}}\left(\alpha_{i}-\mu_{i}-\delta_{2}\right)^{2}\right\}
\end{aligned}
$$

A partir da distribuição a posteriori conjunta, podemos definir as distribuições condicionais para o algoritmo Gibbs Sampling e, então, responder as questões de interesse.

\subsubsection{Aspectos computacionais}

As distribuições condicionais para $\alpha_{i}, \mu_{i}, \delta_{k}, \sigma_{k}^{2}, i=1, \ldots, 10$ e $k=1,2$ são dadas respectivamente por: 


$$
\begin{aligned}
& \pi\left(\alpha_{i} \mid \alpha_{(i)}, \underset{\sim}{\mu}, \delta_{1}, \delta_{2}, \sigma_{1}^{2}, \sigma_{2}^{2}, D\right) \propto N o r m a l\left[\frac{\sigma_{k}^{2} y_{i}+S_{i}^{2}\left(\mu_{i}+\delta_{k}\right)}{\sigma_{k}^{2}+S_{i}^{2}}, \frac{S_{i}^{2} \sigma_{k}^{2}}{\sigma_{k}^{2}+S_{i}^{2}}\right] \\
& \pi\left(\mu_{i} \mid \underset{-}{\alpha}, \mu_{(i)}, \delta_{1}, \delta_{2}, \sigma_{l}^{2}, \sigma_{2}^{2}, D\right) \propto \operatorname{Normal}\left(\alpha_{i}-\delta_{k}, \sigma_{k}^{2}\right) \\
& \pi\left(\delta_{k} \mid \underset{\sim}{\alpha}, \mu, \delta_{(k)}, \sigma_{1}^{2}, \sigma_{2}^{2}, D\right) \propto \operatorname{Normal}\left(\frac{\sigma_{k}^{2} a_{k}+b_{k} I_{k} \bar{\alpha}_{k}-b_{k} I_{k} \bar{\mu}_{k}}{\sigma_{k}^{2}+b_{k} I_{k}}, \frac{b_{k} \sigma_{k}^{2}}{\sigma_{k}^{2}+b_{k} I_{k}}\right) \\
& \text { onde, } \bar{\alpha}_{k}=\frac{\sum_{i=1}^{I_{k}} \alpha_{1}}{I}, \bar{\mu}_{k}=\frac{\sum_{i=1}^{l_{k}} \mu_{i}}{I} \text { e } I=10 \\
& \pi\left(\sigma_{k}^{2} \mid \underset{\sim}{\alpha} \underset{\sim}{\mu} \delta_{1}, \delta_{2}, \sigma_{(k)}^{2}, D\right) \propto \text { Gama Inversa }\left(\frac{I_{k}}{2}+c_{k}, d_{k}+\frac{\sum_{i=1}^{l_{k}}\left(\alpha_{1}-\mu_{i}-\delta_{k}\right)^{2}}{2}\right)
\end{aligned}
$$

\subsubsection{Resultados}

Utilizando a modelagem descrita na Seção 5.3, obtivemos os resultados dado na Tabela 8. 
Tabela 8: Médias a posteriori e intervalos de credibilidade para cada um dos parâmetros.

\begin{tabular}{ccc}
\hline Parâmetros & Médias a posteriori & Intervalos de credibilidade (95\%) \\
\hline$\mu_{1}$ & 0.1170 & $(-3.0102 ; 3.0389)$ \\
$\mu_{2}$ & -0.4980 & $(-4.0757 ; 3.0682)$ \\
$\mu_{3}$ & 0.0645 & $(-2.8764 ; 3.0956)$ \\
$\mu_{4}$ & 0.9129 & $(-1.7100 ; 3.9364)$ \\
$\mu_{5}$ & 0.5563 & $(-2.2650 ; 3.5617)$ \\
$\mu_{6}$ & 0.4496 & $(-2.4722 ; 3.3462)$ \\
$\mu_{7}$ & 0.8750 & $(-2.8660 ; 4.1967)$ \\
$\mu_{8}$ & 0.2526 & $(-2.7361 ; 3.1907)$ \\
$\mu_{9}$ & 0.5349 & $(-2.5361 ; 3.6914)$ \\
$\mu_{10}$ & 0.4241 & $(-2.2827 ; 3.3034)$ \\
$\sigma_{1}^{2}$ & 1.4000 & $(0.3155 ; 5.7928)$ \\
$\sigma_{2}^{2}$ & 1.3627 & $(0.3105 ; 5.4323)$ \\
$\alpha_{1}$ & 0.1774 & $(-0.7884 ; 1.1035)$ \\
$\alpha_{2}$ & -0.4803 & $(-1.6397 ; 0.6235)$ \\
$\alpha_{3}$ & 0.0798 & $(-0.6578 ; 0.8308)$ \\
$\alpha_{4}$ & 0.8824 & $(-0.1640 ; 1.9716)$ \\
$\alpha_{5}$ & 0.5437 & $(0.0588 ; 1.0507)$ \\
$\alpha_{6}$ & 0.4595 & $(-0.0064 ; 0.9025)$ \\
$\alpha_{7}$ & 0.9283 & $(-0.6862 ; 2.5555)$ \\
$\alpha_{8}$ & 0.2073 & $(0.0059 ; 0.4007)$ \\
$\alpha_{9}$ & 0.5267 & $(-0.0566 ; 1.0869)$ \\
$\alpha_{10}$ & 0.4869 & $(0.1008 ; 0.8496)$ \\
$\delta_{2}$ & 0.0572 & $(-2.1062 ; 2.1034)$ \\
& 0.0190 & $(-1.8522 ; 1.7789)$ \\
\hline \hline
\end{tabular}

Analisando os resultados da Tabela 8 , notamos que $\sigma_{1}^{2}$ e $\sigma_{2}^{2}$ estão muito próximos indicando que os dois grupos se comportam praticamente da mesma forma com respeito a variabilidade.

Através de uma média geral dos $\mu_{i}{ }^{\prime} s$ dada por $M . P_{\text {geral }}=\frac{\sum_{i=1}^{I} \mu_{i} n_{i}}{\sum_{i=1}^{I} n_{i}}$, onde $I=10$, temos que $M . P_{\text {geral }}>0,\left(M \cdot P_{\text {geral }}=0.3296 \mathrm{~cm}\right)$ indicando que se analisarmos o estudo como um todo, em média o novo tratamento pode levar a maior durabilidade dos sapatos, mas nada significativo. 
Podemos dizer também que uma afirmação positiva em relação a existir uma diferença entre classes sociais quando analisamos o desgaste das solas de sapatos, não pode ser tomada, ou seja, não existe diferenças significativas entre os grupos, pois os efeitos aleatórios $\delta_{1}$ e $\delta_{2}$, se encontram bem próximos de zero e seus intervalos de credibilidade podem confirmar a não significância.

\subsubsection{Análise gráfica}

Para uma melhor visualização dos resultados obtidos, podemos fazer uma análise gráfica.

A Figuras 8 mostra a forma da distribuição marginal para cada um dos parâmetros.
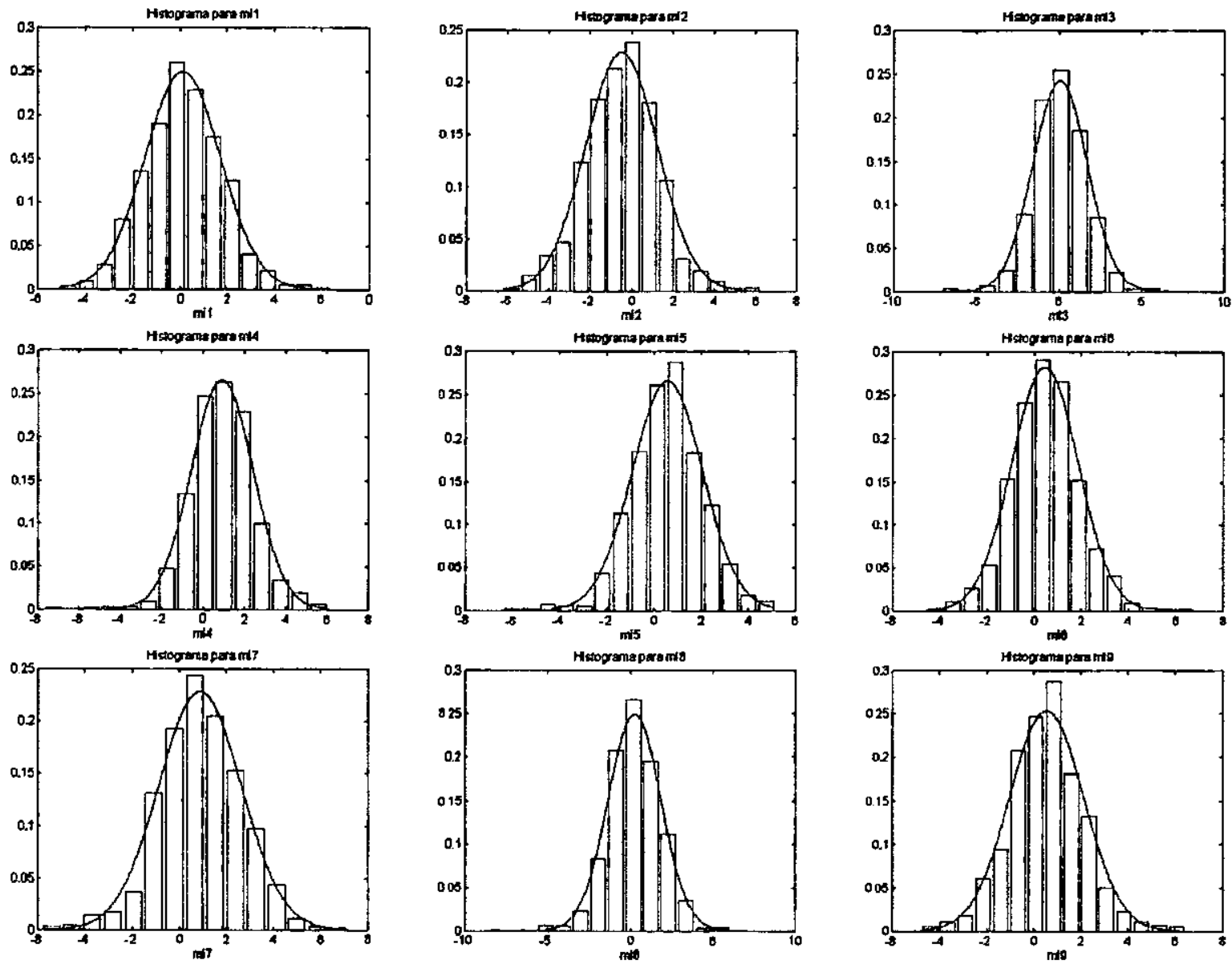

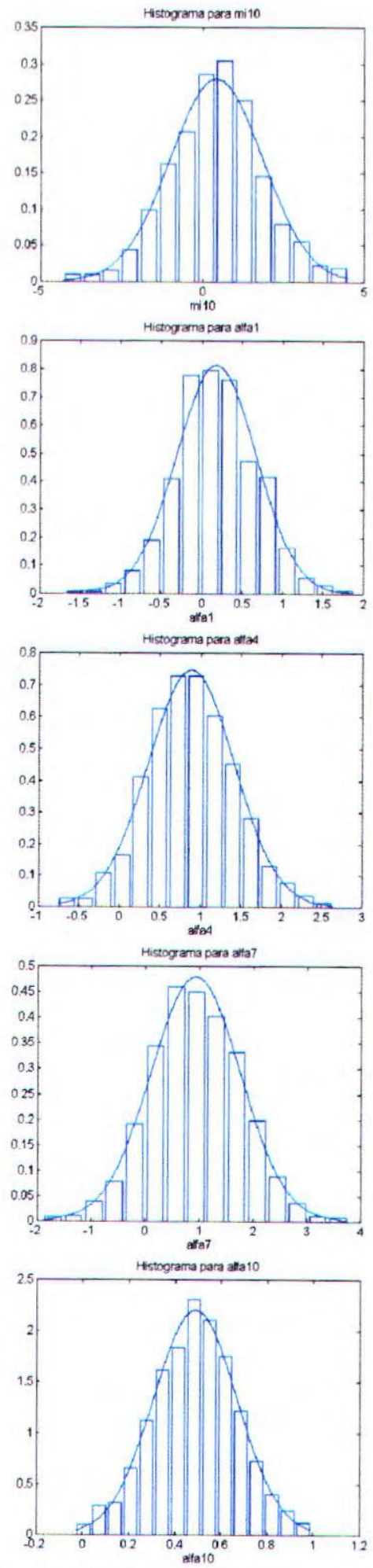

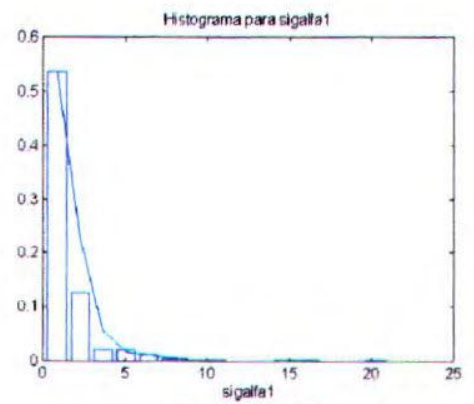

Histograma para atta?

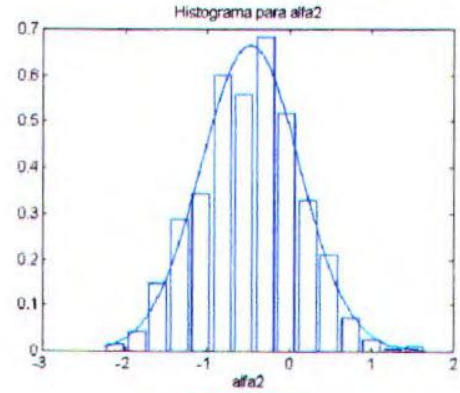

Histograma para altas

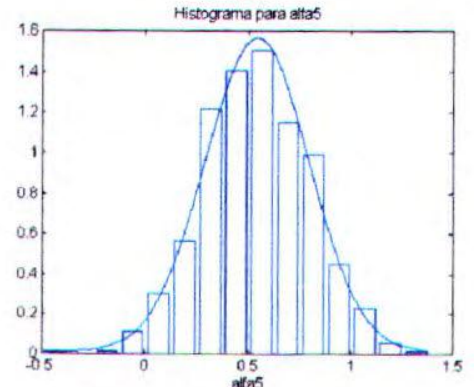

Histograma para atas
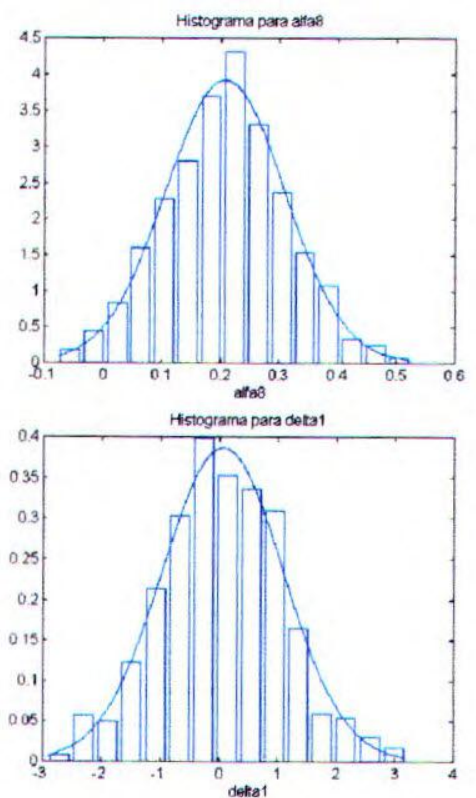

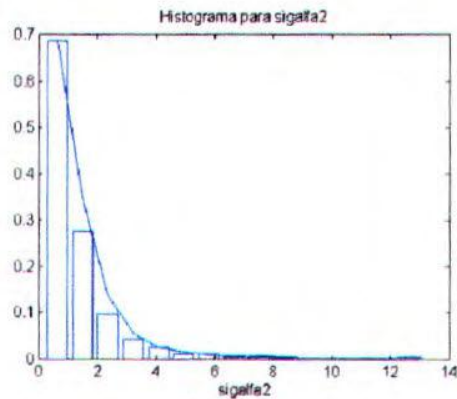

Histograma pare atta:

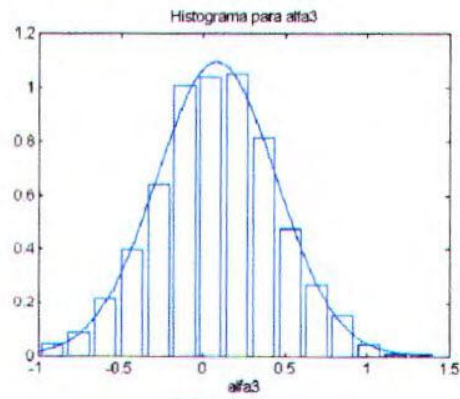

Histograma para attae

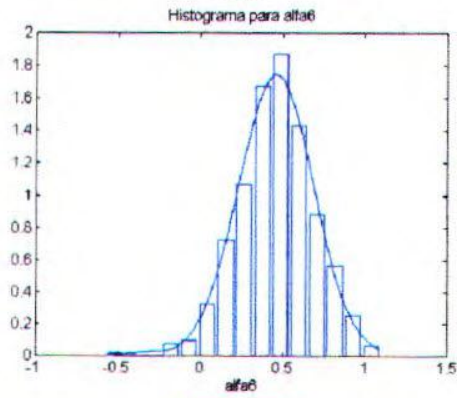

Histograma para atta:

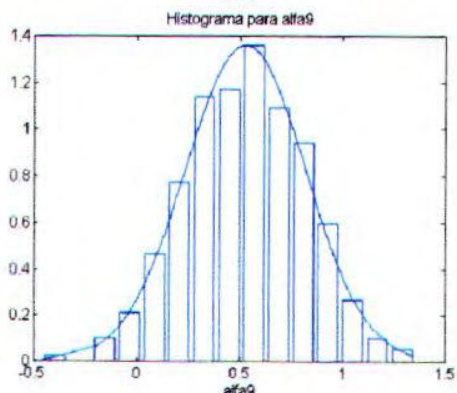

Hstograma para deta2

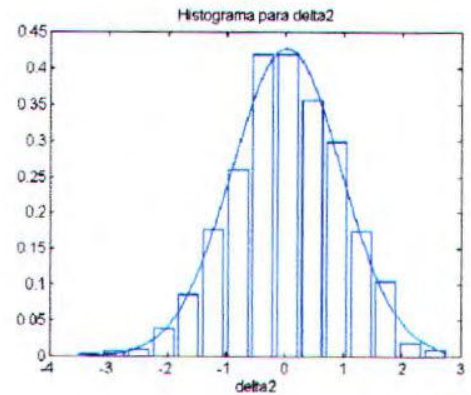

Figura 8: Distribuições marginais a posteriori para $\alpha_{1}, \ldots, \alpha_{1}, \mu_{1}, \ldots, \mu_{1}, \delta_{1}, \delta_{2}, \sigma_{1}^{2}, \sigma_{2}^{2}$, respectivamente. 
A Figura 9 mostra graficamente a convergência dos parâmetros que foi confirmada através do critério de convergência proposto por Gelman e Rubin (1992).
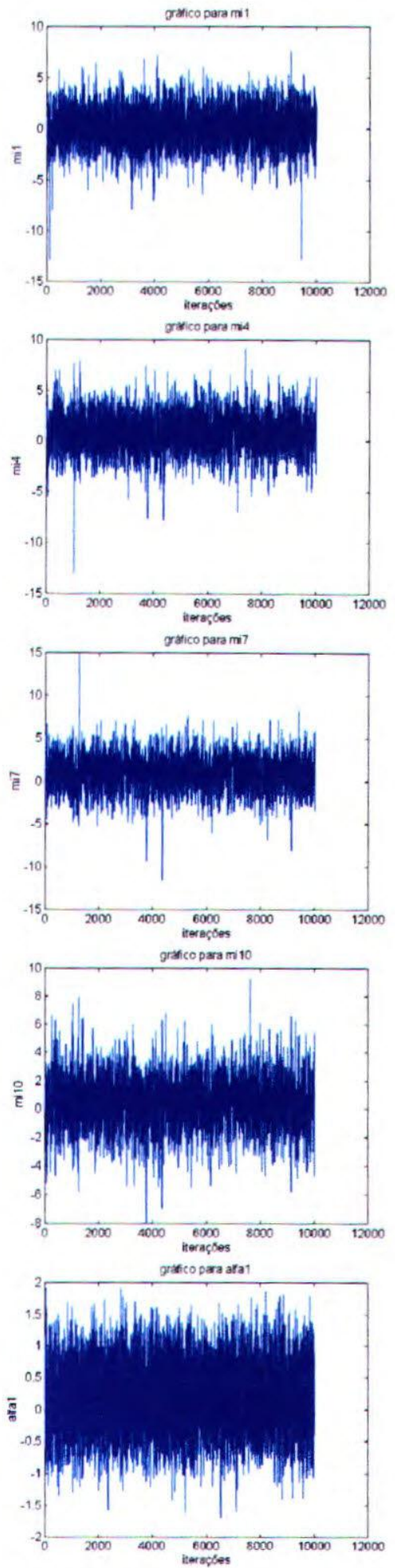
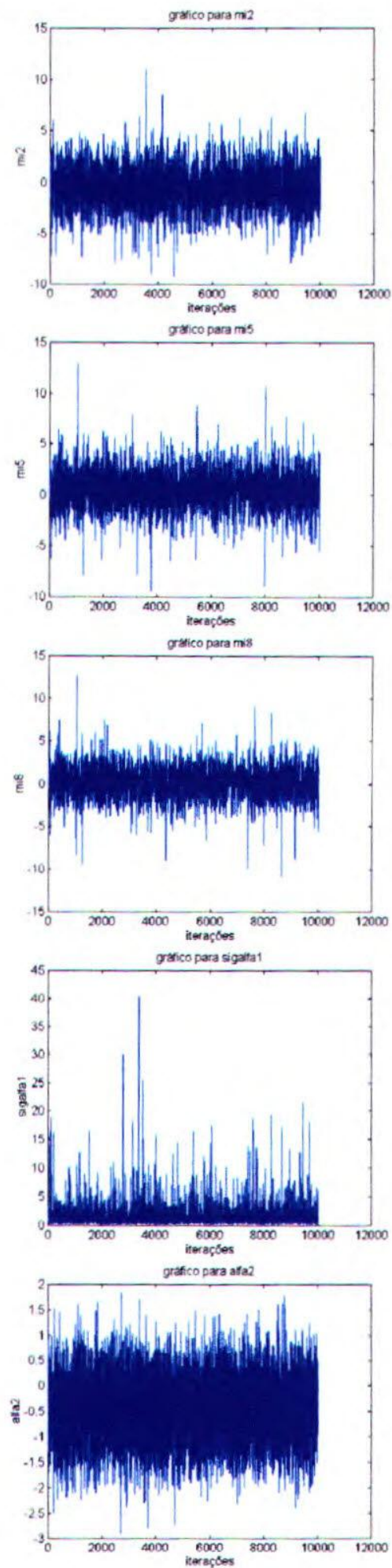
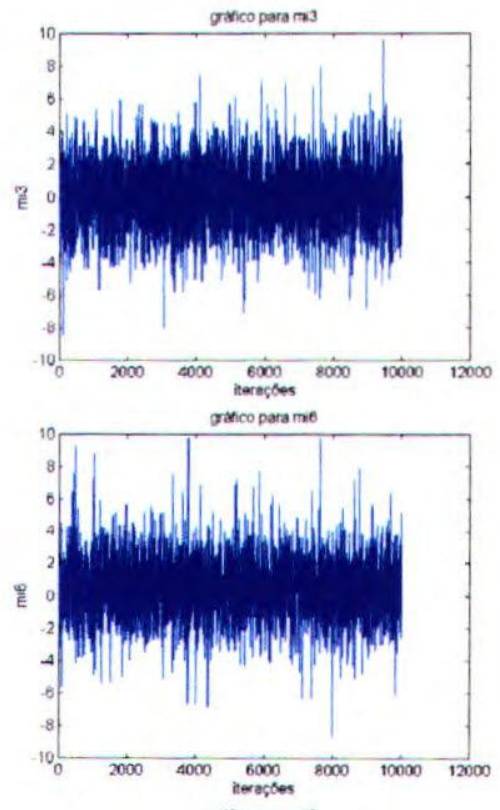
gratico para ms
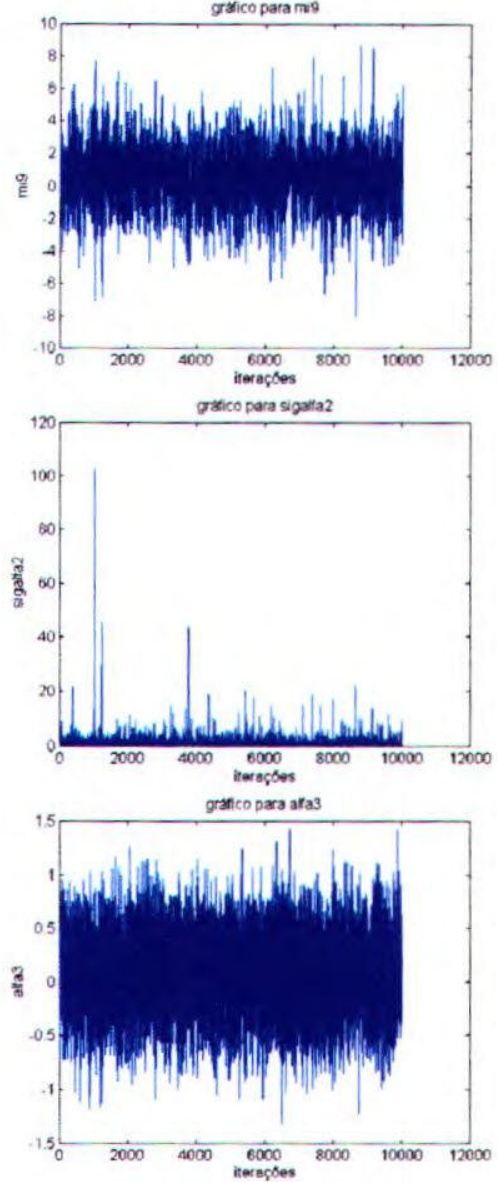

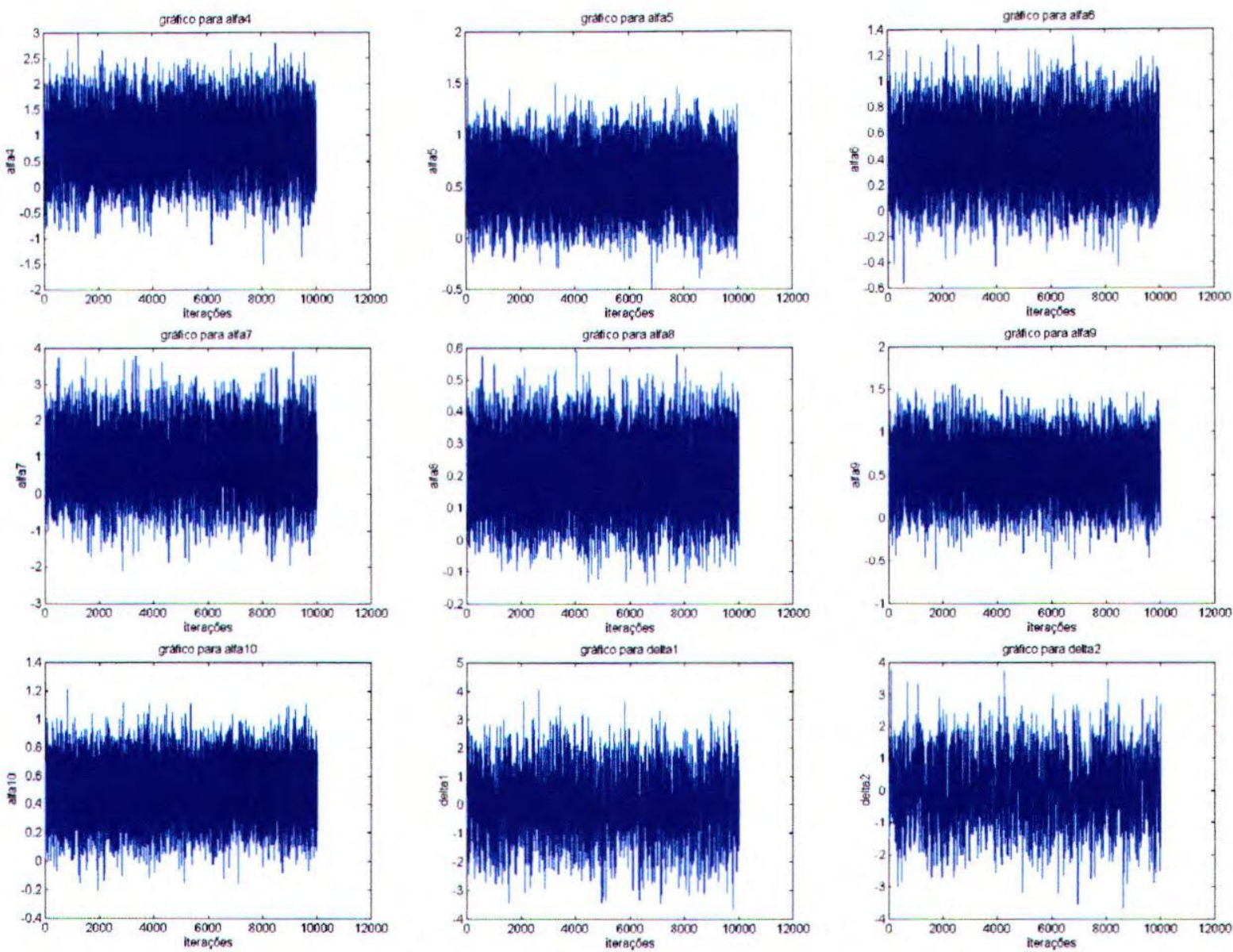

Figuras 9: Gráficos com todos os valores gerados para $\alpha_{1}, \ldots, \alpha_{1}, \mu_{1}, \ldots, \mu_{1}, \delta_{1}, \delta_{2}, \sigma_{1}^{2}, \sigma_{2}^{2}$, respectivamente.

Esta parte computacional também foi desenvolvida utilizando o software MATLAB, gerando cinco cadeias de 2000 valores cada, totalizando uma amostra de 10000 valores gerados. Desprezando $25 \%$ dos valores iniciais gerados por cada cadeia e selecionando os $75 \%$ restantes de 10 em 10, obtivemos uma amostra selecionada de 750 valores, pretendendo com isso assegurar a suposição de independência.

O critério proposto por Gelman e Rubin (1992) para a verificação da convergência foi aplicado e os valores obtidos estão descritos na Tabela 9 
Tabela 9: Valores usados como indicador de convergência, obtidos aplicando o critério de Gelman e Rubin (1992)

\begin{tabular}{ll}
\hline Parâmetros & Valores Obtidos $(\sqrt{\hat{\boldsymbol{R}}})$ \\
\hline$\mu_{1}$ & 0.9971 \\
$\mu_{2}$ & 1.0040 \\
$\mu_{3}$ & 1.0069 \\
$\mu_{4}$ & 1.0013 \\
$\mu_{5}$ & 1.0019 \\
$\mu_{6}$ & 1.0012 \\
$\mu_{7}$ & 1.0057 \\
$\mu_{8}$ & 1.0021 \\
$\mu_{9}$ & 0.9991 \\
$\mu_{10}$ & 1.0054 \\
$\sigma_{1}^{2}$ & 1.0149 \\
$\sigma_{2}^{2}$ & 1.0008 \\
$\alpha_{1}$ & 1.0074 \\
$\alpha_{2}$ & 1.0077 \\
$\alpha_{3}$ & 1.0006 \\
$\alpha_{4}$ & 0.9986 \\
$\alpha_{5}$ & 1.0045 \\
$\alpha_{6}$ & 1.0068 \\
$\alpha_{7}$ & 1.0000 \\
$\alpha_{8}$ & 1.0046 \\
$\alpha_{9}$ & 1.0087 \\
$\delta_{2}$ & 1.0000 \\
\hline \hline & 1.0016 \\
\hline & 0.9999 \\
\hline
\end{tabular}

Analisando os resultados da Tabela 9, podemos concluir pela convergência dos parâmetros. 


\subsection{Escolha e verificação do ajuste do modelo}

Analisando os modelos através da densidade preditiva ordenada definida em (2.6), e utilizando o critério proposto na Seção 2.5, encontramos $c_{l}=4.2485 \times 10^{-42}$ e $c_{2}=5.0779 \times 10^{-43}$, portanto, o modelo escolhido dentre os dois analisados neste Capítulo foi o modelo 1. Pudemos concluir que este modelo estava adequado utilizando o procedimento proposto na Seção 2.6.1, onde o valor de $\hat{p}_{v}$ (definido em (2.11)) usando a estatística de teste definida em (2.10) foi 0.55 . Portanto, podemos decidir pela não rejeição do modelo, já que $0.05<\hat{p}_{v}<0.95$. 


\section{CAPÍTULO 6 - RELAÇÃO ENTRE O USO DE HORMÔNIO E CÂNCER EM MULHERES: UMA APLICAÇÃO DE META- ANÁLISE SOB UM ENFOQUE BAYESIANO}

\subsection{Introdução}

Nos últimos anos tem havido muita pesquisa médica para avaliar a possível associação entre a incidência de câncer nos seios, ovários e útero e o uso de hormônios (ver por exemplo, Adami,1992; Kaufman, 1991; ou Brinton \& Schairer, 1993)

Câncer nos seios útero e ovários representam mais que um quarto dos casos de mortes entre mulheres (Kelsey \& Whittemore, 1994). Nos Estados Unidos entre 1960 e 1975, a taxa de câncer endometrial mostrou um aumento sem precedentes ao mesmo tempo em que o uso de hormônio (estrógeno) durante este periodo também aumentou bruscamente (Jick, Walker, e Jick, 1993). Daí veio a suspeita de que o uso de estrógeno poderia estar contribuindo para o desenvolvimento de câncer endometrial.

Em 1975, foram publicados dois artigos os quais mostraram grandes riscos relativos (de 5 a 7 vezes) para o câncer endometrial entre mulheres que já fizeram uso de estrógeno e mulheres que nunca foram usuárias. (Ziel \& Finkle, 1975; Smith et al, 1975)

O efeito desta publicação entre 1975 e 1980, foi a redução em 50\% das receitas preparadas com estrógeno sem anticoncepcionais, ao mesmo tempo, a incidência de câncer endometrial também diminuiu (Jick, Walker, e Jick, 1993).

Mais recentemente, o efeito preventivo do estrógeno sob moléstias cardiovasculares e osteoporose, levou, outra vez, a um aumento no uso de estrógeno. 
Hoje, por volta de $32 \%$ das mulheres americanas com idade entre 50 e 65 anos usam estrógeno para menopausa, a reposição de estrógeno é, como colocou Adami em 1992, "potencialmente um dos meios mais importantes de prevenir a maioria das causas de morbidez e mortalidade de mulheres mais velhas".

Apesar do fato de que estrógeno pode gerar câncer endometrial ter sido geralmente aceito, conclusões quantitativas a respeito dos efeitos do uso do estrógeno no risco de câncer no seio permanece indescritivel. Meta-análise tem sido usada para tentar resolver este dilema.

Recentemente foram desenvolvidas algumas meta-análises mostrando o risco relativo de usuários de estrógeno com respeito a câncer nos seios estar acima de 1.06 , onde risco relativo é a medida da força da associação entre estrógeno e câncer e pode ser obtido da seguinte forma (ver Fleiss, 1973):

Definindo:

$A=$ pacientes com câncer;

$\bar{A}=$ pacientes sem câncer;

$B=$ pacientes recebendo estrógeno;

$\bar{B}=$ pacientes não recebendo estrógeno.

A partir das definições acima, temos o seguinte quadro,

\begin{tabular}{|c|cc|c|}
\hline & $A$ & $\bar{A}$ & Total \\
\hline$B$ & $a$ & $b$ & $a+b$ \\
$\bar{B}$ & $c$ & $d$ & $c+d$ \\
\hline Total & $a+c$ & $b+d$ & $a+b+c+d$ \\
\hline
\end{tabular}

onde, $a$ é o número de casos expostos, $b$ é o número de controles expostos, $c$ é o número de casos não expostos e $d$ é o número de controles não expostos.

Assim, definimos o risco para dois grupos. O primeiro é definido a partir de pacientes que são usuárias de estrógeno e o segundo é definido a partir de pacientes que não são usuárias de estrógeno, ou seja, 
pacientes usuárias de estrógeno: $O_{B}=\frac{P(A \mid B)}{P(\bar{A} \mid B)}$

pacientes não usuárias de estrógeno: $O_{\bar{B}}=\frac{P(A \mid \bar{B})}{P(\bar{A} \mid \bar{B})}$

Uma medida de associação entre $O_{B}$ e $O_{\bar{B}}$ será denotada por:

$o=\frac{O_{B}}{O_{\bar{B}}}=\frac{p_{11} / p_{12}}{p_{21} / p_{22}}=\frac{p_{11} p_{22}}{p_{12} p_{21}}$

onde

$\hat{p}_{11}=a /$ n. ; $\hat{p}_{12}=b /$ n. ; $\hat{p}_{21}=c /$ n.; $\hat{p}_{22}=d / n .$. e $n .=a+b+c+d$

Portanto, um estimador desta associação, chamado de risco relativo $(R R)$, é dado por:

$$
R R=\hat{o}=\frac{(a / n . .)(d / n . .)}{(b / n . .)(c / n . .)}=\frac{a d}{b c}
$$

Se $R R>1$, isso indica que a chance de uma mulher ter câncer (seios, útero e ovários) é maior para as usuárias de estrógeno.

Para este tipo de problema, a modelagem hierárquica Bayesiana descreve de forma natural os efeitos de covariáveis e outras características do modelo. Portanto, a motivação é forte para aplicar novos modelos Bayesianos para a investigação da ligação sugerida entre uso de estrógeno e câncer nos seios e endométrio.

O objetivo principal deste estudo é tirar conclusões quantitativas relacionadas principalmente aos efeitos do estrógeno em câncer nos seios, útero e ovários. Porém, os efeitos de outras covariáveis também são analisados para complementar o estudo. 
Em geral os estudos são feitos por vários pesquisadores independentes, portanto, o uso de meta-análise para verificar se existe relação entre estrógeno e câncer desse tipo é adequado.

Interesse do pesquisador:

- Há alguma associação entre o uso de estrógeno e câncer nos seios ou endométrio? Isto é, “a hipótese do modelo de nulidade é rejeitada?"

- Há alguma covariável associada com o risco de câncer? Isto é, para uma dada covariável $m$, a posteriori para $\mu_{m}$ é diferente de zero?

- Há alguma diferença quantificável entre os estudos com dados perdidos? Isto é, $\delta$ indica algum efeito significativo entre os estudos com dados perdidos?

\subsection{Relação entre o uso de estrógeno e a incidência de câncer no útero e ovários}

Para sabermos se existe alguma relação entre estrógeno e câncer endometrial, vamos analisar os dados descritos na Tabela 10 (dados em Larose \& Dey, 1995 b):

Tabela 10: Dados para laboratórios diferentes (incidência de câncer de útero e ovários)

\begin{tabular}{|c|c|c|c|c|c|c|c|c|}
\hline $\begin{array}{c}\text { Laborató- } \\
\text { rios }\end{array}$ & Resposta & \multicolumn{3}{|c|}{$\begin{array}{c}\text { Duração do Tratamento } \\
\text { com Estrógeno (em meses) }\end{array}$} & \multicolumn{2}{c|}{ Tipo de Estrógeno } & \multicolumn{2}{c|}{ Obesidade } \\
\hline & $\log (\boldsymbol{R R})$ & $<\mathbf{1 2}$ & {$[\mathbf{1 2 , 6 0 )}$} & $\geq \mathbf{6 0}$ & Conjugado & Outros & Não & Sim \\
\hline $\mathbf{1}$ & 2.08 & 22 & 55 & 56 & 137 & 40 & 186 & 129 \\
\hline $\mathbf{2}$ & -0.06 & 68 & 12 & 11 & 68 & 134 & 584 & 126 \\
\hline $\mathbf{3}$ & 0.76 & 49 & 70 & 121 & 334 & 122 & 405 & 146 \\
\hline $\mathbf{4}$ & 1.58 & 18 & 25 & 39 & 74 & 10 & - & - \\
\hline $\mathbf{5}$ & 0.87 & 11 & 17 & 49 & - & - & 722 & 140 \\
\hline $\mathbf{6}$ & 0.71 & 57 & 85 & 39 & - & - & 991 & 188 \\
\hline $\mathbf{7}$ & 1.24 & 26 & 61 & 98 & - & - & 596 & 190 \\
\hline $\mathbf{8}$ & 1.99 & 8 & 17 & 27 & 54 & 45 & - & - \\
\hline
\end{tabular}

Na Tabela 10, observamos que para vários laboratórios há informação perdida para as covariáveis. Para analisar estes dados; podemos considerar o uso de meta-análise sob o enfoque Bayesiano (ver por exemplo, DuMouchel \& Harris, 1983; ou Larose \& Dey, 1995) 
Assumir o seguinte modelo para analisar os dados da Tabela 10:

$$
\underset{\sim}{y}=X+\underset{\sim}{\beta} \delta+\varepsilon
$$

onde $y$ é um vetor (nxl) das respostas dadas por $y=\log (R R)$; $X$ é uma matriz (nx3) de covariáveis dada por,

$$
X=\left(\begin{array}{lll}
x_{11} & x_{21} & x_{31} \\
x_{12} & x_{22} & x_{32} \\
\cdot & \cdot & \cdot \\
\cdot & \cdot & \cdot \\
\cdot & \cdot & \cdot \\
x_{1 n} & x_{3 n} & x_{3 n}
\end{array}\right)=\left(x_{1}, x_{2}, x_{3}\right)
$$

onde $x_{1}$ é o vetor da covariável denotando a proporção do tempo de tratamento maior ou igual à 60 meses; $x_{2}$ é o vetor da covariável denotando a proporção de usuárias de estrógeno conjugado e $x_{3}$ é o vetor da covariável denotando a proporção de obesas em cada estudo; $\beta$ é o vetor ( $\left.3 \times 1\right)$ de parâmetros de regressão $\beta_{m}, m=1,2,3 ; \delta$ é o vetor $(2 \times 1)$ de parâmetros $\delta$, é uma variável dummy que mede o efeito dos dois grupos diferentes (com informação completa nas covariáveis, ou não); $w$ é um vetor (nxl) dado por,

$$
w=\left(\begin{array}{l}
w_{11} \\
w_{12} \\
\\
w_{1 n}
\end{array}\right)
$$

onde $w_{i}=1(0) ; i=1,2, \ldots, n$ indica informação completa (ou não) para as covariáveis, e $\varepsilon$ é o vetor de erros com elementos $\varepsilon_{i}, i=1,2, \ldots, n$. 
Assumir que as variáveis aleatórias $\varepsilon_{i}, i=1,2, \ldots, n$ sejam independentes, identicamente distribuidas com distribuição normal com média zero e variância $\sigma^{2}$.

Associado ao modelo (6.2) com os dados da Tabela 10, temos:

$$
X=\left(\begin{array}{ccc}
0.42 & 0.77 & 0.41 \\
0.12 & 0.33 & 0.18 \\
0.50 & 0.73 & 0.26 \\
0.47 & 0.88 & - \\
0.64 & - & 0.16 \\
0.21 & - & 0.16 \\
0.53 & - & 0.24 \\
0.52 & 0.54 & -
\end{array}\right) ; \quad \boldsymbol{w}=\left(\begin{array}{l}
1 \\
1 \\
1 \\
0 \\
0 \\
0 \\
0 \\
0
\end{array}\right)
$$

Para as observações perdidas em $X$, podemos considerar um estimador simplificado dado pela média das observações completas em cada vetor $x_{j}, j=1,2,3$; ou usar um método de simulação para gerar essas observações perdidas.

Larose e Dey (1995 b) consideram uma extensão do modelo de componentes de variância hierárquico de DuMouchel e Harris (1983) para analisar os dados da Tabela 10, usando métodos de Monte Cario em cadeias de Markov (MCMC) para uma análise Bayesiana.

Nesta dissertação assumimos o modelo (6.2) e uma análise Bayesiana não-hierárquica usando o algoritmo Gibbs Sampling (ver por exemplo, Gelfand \& Smith, 1990) para determinar as quantidades a posteriori de interesse.

Além do uso de meta-análise sob o enfoque Bayesiano para relacionar o uso de hormônio (estrógeno) e a incidência de câncer no útero e ovários, também consideramos o modelo (6.2) para analisar um conjunto de dados de vários institutos de pesquisa introduzido por Larose e Dey (1995 b) relacionando a incidência de câncer nos seios e o uso de hormônios associados com vários fatores de risco. 


\subsection{Uma análise Bayesiana para os dados da Tabela 10}

Como visto anteriormente, um modelo simplificado para analisar os dados da Tabela 10 é dada por,

$$
\underline{y}=X \beta+\underset{\sim}{w} \delta+\varepsilon
$$

\subsubsection{Função de verossimilhança}

A função de verossimilhança para os parâmetros $\beta, \delta$ e $\sigma^{2}$ do modelo (6.2) é dada por,

$$
\begin{aligned}
L\left(\beta, \delta, \sigma^{2}\right) & =\coprod_{i=1}^{n} \frac{1}{\sqrt{2 \pi \sigma^{2}}} \exp \left\{-\frac{1}{2 \sigma^{2}}\left(\underset{\sim}{\left.\mathcal{y}-(X \beta-w \delta)^{2}\right)}\right)\right\} \\
& \propto\left(\sigma^{2}\right)^{-n / 2} \exp \left\{-\frac{1}{2 \sigma^{2}} \sum_{i=1}^{n}\left(y_{i}-x_{1 i} \beta_{1}-x_{2 i} \beta_{2}-x_{3 i} \beta_{3}-w_{i} \delta\right)^{2}\right\}
\end{aligned}
$$

\subsubsection{Distribuição a priori}

Para uma análise Bayesiana, assumir as seguintes distribuições a priori para os parâmetros:

$\delta \sim N\left(0, a^{2}\right) ; a$ conhecido;

$\beta_{m} \sim N\left(0, b_{-m}^{2}\right) ; m=1,2,3 ; b_{m}$ conhecidos;

$\sigma^{2} \sim I G(c, d) ; c, d$ conhecidos,

onde $N\left(a, b^{2}\right)$ denota uma distribuição normal com média $a$ e variância $b^{2}$ e $I G(c, d)$ denota uma distribuição gama inversa com média $d /(c-1)$ e variância $d^{2} /(c-l)^{2}(c-2)$. Também assumir independência a priori entre os parâmetros 
Assumindo independência a priori entre os parâmetros, e considerando $b_{1}=b_{2}=b_{3}=b$, podemos expressar a distribuição a priori conjunta para os parâmetros $\beta, \delta$ e $\sigma^{2}$ por,

$$
\pi\left(\beta, \delta, \sigma^{2}\right) \propto \exp \left\{-\frac{1}{2 a^{2}}\left(\delta^{2}\right)\right\} \exp \left\{-\frac{1}{2 b^{2}}\left(\beta_{1}^{2}+\beta_{3}^{2}+\beta_{3}^{2}\right)\right\}\left(\sigma^{2}\right)^{-(c+1)} \exp \left\{-\frac{d}{\sigma^{2}}\right\}
$$

\subsubsection{Distribuição a posteriori conjunta}

Partindo de (6.6) e (6.7) temos que a distribuição a posteriori conjunta para $\beta, \delta$ e $\sigma^{2}$ é dada por:

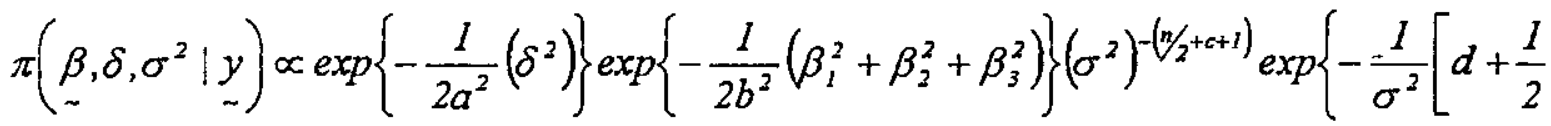

$$
\begin{aligned}
& \left.\left.\sum_{i=1}^{n}\left(y_{t}-x_{l i} \beta_{1}-x_{3 i} \beta_{2}-x_{3 i} \beta_{3}-w_{i} \delta\right)^{2}\right]\right\}
\end{aligned}
$$

Observamos que $\sum_{i=1}^{n}\left(y_{1}-x_{1 i} \beta_{1}-x_{2 i} \beta_{2}-x_{3 i} \beta_{3}-w_{i} \delta\right)^{2}$ pode ser escrito como $(\underset{\sim}{y-X} \underset{\sim}{\beta} \underset{\sim}{w})^{\prime}(\underset{\sim}{y-X} \underset{\sim}{\beta}-\underset{\sim}{\delta})$, onde

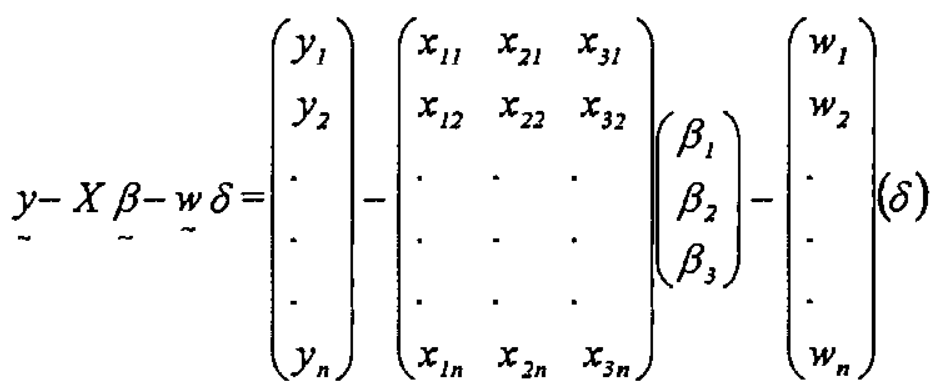

Desenvolvendo a expressão $(\underset{\sim}{y-X} \underset{\sim}{\beta-w} \delta)^{\prime}(\underset{\sim}{y-X \beta-\underset{\sim}{w} \delta})$, temos, 


$$
\begin{aligned}
& (\underset{\sim}{y-X} \underset{\sim}{\beta}-\underset{\sim}{w} \delta)^{\prime}(\underset{\sim}{y}-\underset{\sim}{\beta}-\underset{\sim}{w} \delta)=\left(\underset{\sim}{y^{\prime}}-\beta^{\prime} X^{\prime}-\delta^{\prime} \underset{\sim}{w}\right)(\underset{\sim}{y}-X \underset{\sim}{\beta}-\underset{w}{w} \delta)=
\end{aligned}
$$

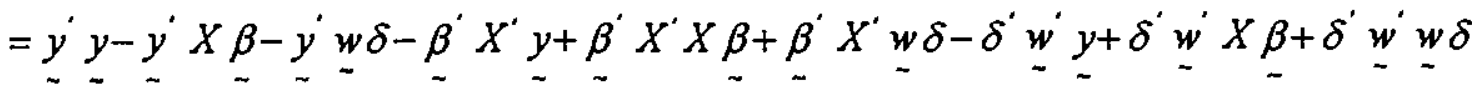

onde $y^{\prime}, w^{\prime}$ e $\beta^{\prime}$ denotam a transposta do vetor $y, \underline{w}$ e $\beta$ respectivamente, e $X^{\prime}$ denota a transposta da matriz $X$.

Assim podemos rescrever a distribuição a posteriori conjunta dada em (6.9) por,

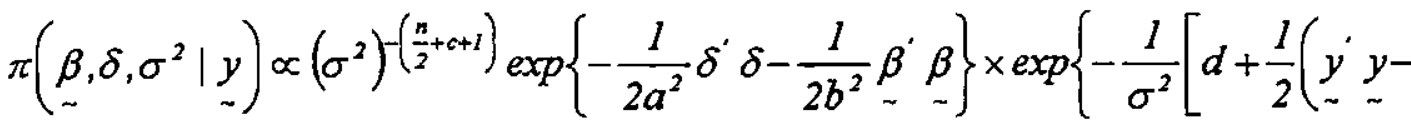

$$
\begin{aligned}
& \left.\left.\left.-2 \underline{y^{\prime}} X \underline{\beta}-2 \underset{\sim}{y^{\prime}} \underset{\sim}{w} \delta+\beta^{\prime} X^{\prime} X \underline{\beta}+2 \underset{\sim}{\beta} X^{\prime} \underset{\sim}{w} \delta+\delta^{\prime} \underset{\sim}{w^{\prime}} \underset{\sim}{w} \delta\right)\right]\right\}
\end{aligned}
$$

\subsubsection{Aspectos computacionais}

A partir da distribuição a posteriori conjunta dada em (6.11), as distribuições condicionais necessárias para o algoritmo Gibbs Sampling são dadas por.

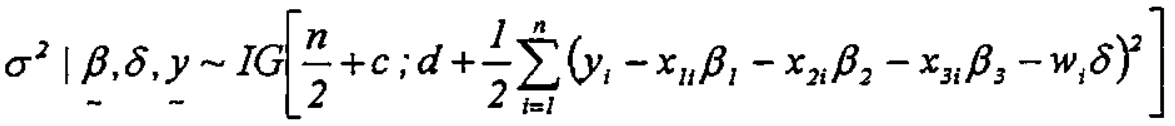

$$
\begin{aligned}
& \pi\left(\underset{\sim}{\beta} \mid \sigma^{2}, \delta, \underset{\sim}{y}\right) \propto \exp \left\{-\frac{1}{2 b^{2}} \beta_{\sim}^{\prime} \beta+\frac{1}{\sigma^{2}} y_{\sim}^{\prime} X \underset{\sim}{\beta}-\frac{1}{2 \sigma^{2}} \beta_{\sim}^{\prime} X^{\prime} X \beta-\frac{1}{\sigma^{2}} \beta_{\sim}^{\prime} X^{\prime} \underset{\sim}{w} \delta\right\} \\
& \pi\left(\delta \mid \sigma^{2}, \beta, y\right) \propto \exp \left\{-\frac{1}{2 a^{2}} \delta^{\prime} \delta+\frac{1}{\sigma^{2}} y_{\sim}^{\prime} \underset{\sim}{w} \delta-\frac{1}{2 \sigma^{2}} \delta^{\prime} \underset{\sim}{w} \underset{\sim}{w} \delta-\frac{1}{\sigma^{2}} \beta^{\prime} X^{\prime} w \delta\right\}
\end{aligned}
$$

Usando alguns resultados para $\pi\left(\underset{\sim}{\beta} \mid \sigma^{2}, \delta, y\right)$ tais como os descritos abaixo, 
(1) $y^{\prime} X \beta=a_{1} \beta_{1}+a_{2} \beta_{2}+a_{3} \beta_{3}$

onde $a_{j}=\sum_{i=1}^{n} y_{i} x_{j i}, j=1,2,3$

(2) $\beta^{\prime} X^{\prime} X \beta=b_{11} \beta_{1}^{2}+2 b_{12} \beta_{1} \beta_{2}+b_{22} \beta_{3}^{2}+2 b_{3 l} \beta_{1} \beta_{3}+2 b_{23} \beta_{2} \beta_{3}+b_{33} \beta_{3}^{2}$

onde $b_{j k}=\sum_{i=1}^{n} x_{j i} x_{k i}, j, k=1,2,3$

(3) $\beta^{\prime} X^{\prime} \underset{\sim}{w} \delta=c_{1} \beta_{1} \delta+c_{2} \beta_{2} \delta+c_{3} \beta_{3} \delta$

onde $c_{j}=\sum_{i=1}^{n} x_{j i} w_{i}, j=1,2,3$

podemos rescrever $\pi\left(\underset{\sim}{\beta} \mid \sigma^{2}, \delta, y\right)$ por,

$$
\begin{aligned}
\pi\left(\underset{\sim}{\beta} \mid \sigma^{2}, \delta, \underset{\sim}{y}\right) \propto & \exp \left\{-\frac{1}{2 b^{2}}\left(\beta_{1}^{2}+\beta_{2}^{2}+\beta_{3}^{2}\right)+\frac{1}{\sigma^{2}}\left(a_{1} \beta_{1}+a_{2} \beta_{2}+a_{3} \beta_{3}\right)-\right. \\
& -\frac{I}{2 \sigma^{2}}\left(b_{11} \beta_{1}^{2}+2 b_{12} \beta_{1} \beta_{2}+b_{22} \beta_{2}^{2}+2 b_{31} \beta_{1} \beta_{3}+2 b_{23} \beta_{2} \beta_{3}+b_{33} \beta_{3}^{2}\right)- \\
& \left.-\frac{I}{\sigma^{2}}\left(c_{1} \beta_{1} \delta+c_{2} \beta_{2} \delta+c_{3} \beta_{3} \delta\right)\right\}
\end{aligned}
$$

Desta forma, as distribuições condicionais para $\beta=\left(\beta_{1}, \beta_{2}, \beta_{3}\right)$ individualmente, são dadas por:

$$
\begin{aligned}
\pi\left(\beta_{1} \mid \beta_{(1)}, \delta, \sigma^{2}, y\right) \propto & \exp \left\{-\frac{l}{2 b^{2}} \beta_{1}^{2}+\frac{a_{1} \beta_{1}}{\sigma^{2}}-\frac{1}{2 \sigma^{2}}\left(b_{11} \beta_{1}^{2}+2 b_{12} \beta_{1} \beta_{2}+2 b_{13} \beta_{1} \beta_{3}\right)-\right. \\
& \left.-\frac{1}{\sigma^{2}}\left(c_{1} \beta_{1} \delta\right)\right\} \\
\pi\left(\beta_{2} \mid \beta_{(2)}, \delta, \sigma^{2}, y\right) & \propto \exp \left\{-\frac{1}{2 b^{2}} \beta_{2}^{2}+\frac{a_{2} \beta_{2}}{\sigma^{2}}-\frac{I}{2 \sigma^{2}}\left(b_{22} \beta_{2}^{2}+2 b_{12} \beta_{1} \beta_{2}+2 b_{23} \beta_{2} \beta_{3}\right)-\right. \\
& \left.-\frac{1}{\sigma^{2}}\left(c_{2} \beta_{2} \delta\right)\right\}
\end{aligned}
$$




$$
\begin{aligned}
\pi\left(\beta_{3} \mid \beta_{(3)}, \delta, \sigma^{2}, y\right) \propto & \exp \left\{-\frac{1}{2 b^{2}} \beta_{3}^{2}+\frac{a_{3} \beta_{3}}{\sigma^{2}}-\frac{1}{2 \sigma^{2}}\left(b_{33} \beta_{3}^{2}+2 b_{31} \beta_{3} \beta_{1}+2 b_{23} \beta_{2} \beta_{3}\right)-\right. \\
& \left.-\frac{1}{\sigma^{2}}\left(c_{3} \beta_{3} \delta\right)\right\}
\end{aligned}
$$

onde $\beta_{(i)}=\left(\beta_{j}, \beta_{k}\right) ; j \neq i, k \neq i ; i, j, k=1,2,3$;

\section{Definindo,}

$$
\begin{aligned}
& k_{j 1}=\frac{1}{b^{2}}+\frac{b_{i j}}{\sigma^{2}} ; j=1,2,3 ; \\
& k_{12}=\frac{a_{1}}{\sigma^{2}}-\frac{b_{12} \beta_{2}}{\sigma^{2}}-\frac{b_{31} \beta_{3}}{\sigma^{2}}-\frac{c_{1} \delta}{\sigma^{2}} ; \\
& k_{22}=\frac{a_{2}}{\sigma^{2}}-\frac{b_{12} \beta_{1}}{\sigma^{2}}-\frac{b_{23} \beta_{3}}{\sigma^{2}}-\frac{c_{2} \delta}{\sigma^{2}} ; \\
& k_{32}=\frac{a_{3}}{\sigma^{2}}-\frac{b_{31} \beta_{1}}{\sigma^{2}}-\frac{b_{23} \beta_{2}}{\sigma^{2}}-\frac{c_{3} \delta}{\sigma^{2}} ;
\end{aligned}
$$

podemos rescrever as distribuições condicionais para $\beta_{1}, \beta_{2} e \beta_{3}$ como:

$$
\begin{aligned}
& \pi\left(\beta_{1} \mid \beta_{(1)}, \delta, \sigma^{2}, \underset{\sim}{y}\right) \propto \exp \left\{-\frac{k_{11}}{2} \beta_{1}^{2}+k_{12} \beta_{1}\right\}=\exp \left\{-\frac{k_{11}}{2}\left(\beta_{1}-\frac{k_{12}}{k_{11}}\right)^{2}\right\} \\
& \underbrace{\left(\beta_{2} \mid \beta_{(2)}, \delta, \sigma^{2}, y\right)}_{\sim} \underset{\sim}{\exp }\left\{\frac{k_{21}}{2}\left(\beta_{2}-\frac{k_{22}}{k_{21}}\right)^{2}\right\} \\
& \pi\left(\beta_{3} \mid \beta_{(3)}, \delta, \sigma^{2}, y\right) \propto \exp \left\{\frac{k_{31}}{2}\left(\beta_{3}-\frac{k_{32}}{k_{31}}\right)^{2}\right\}
\end{aligned}
$$

Portanto as distribuições condicionais necessárias para o algoritmo Gibbs Sampling para $\beta_{1}, \beta_{2}$ e $\beta_{3}$ são dadas por. 
$\beta_{1} \mid \beta_{(1)}, \delta, \sigma^{2}, \underline{\sim} \sim N\left\{\frac{k_{12}}{k_{l 1}} ; \frac{l}{k_{11}}\right\}$

$\beta_{2} \mid \beta_{(2)}, \delta, \sigma^{2}, \underset{\sim}{y} \sim N\left\{\frac{k_{22}}{k_{21}} ; \frac{1}{k_{21}}\right\}$

$\beta_{3} \mid \beta_{(3)}, \delta, \sigma^{2}, y \sim N\left\{\frac{k_{32}}{k_{31}} ; \frac{1}{k_{31}}\right\}$

Considerar os resultados,

(1) $\delta^{\prime} \delta=\delta^{2}$

(2) $\underset{\sim}{y^{\prime}} \underset{\sim}{\sim} \delta=f \delta$

onde $f=\sum_{i=1}^{n} y_{i} w_{i}$

(3) $\beta^{\prime} X^{\prime} w \delta=c_{1} \beta_{1} \delta+c_{2} \beta_{2} \delta+c_{3} \beta_{3} \delta$

onde $c_{j}=\sum_{i=1}^{n} x_{j} w_{i}, j=1,2,3$

(4) $\delta^{\prime} \underset{\sim}{w} \underset{\sim}{w}=e \delta^{2}$

onde $e=\sum_{i=1}^{n} w_{i}^{2}$

A partir dos resultados obtidos em (6.13) podemos rescrever $\pi\left(\underset{\sim}{\delta} \underset{\sim}{\beta} \sigma^{2}, y\right)$ por,

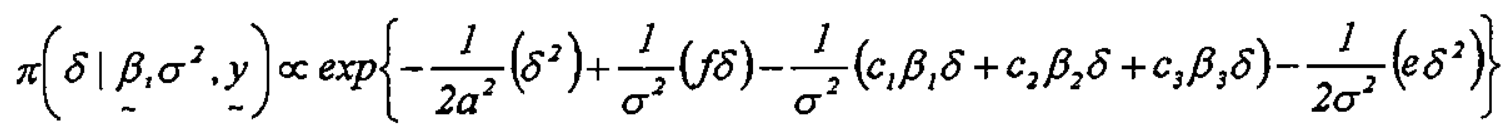


Definindo,

$r_{1}=\frac{l}{a^{2}}+\frac{e}{\sigma^{2}}$

$r_{2}=\frac{f}{\sigma^{2}}-\frac{c_{1} \beta_{1}}{\sigma^{2}}-\frac{c_{2} \beta_{2}}{\sigma^{2}}-\frac{c_{3} \beta_{3}}{\sigma^{2}}$

rescrevemos a distribuição condicional para $\delta$ da seguinte forma:

$$
\pi\left(\delta \mid \underset{\sim}{\beta}, \sigma^{2}, \underset{\sim}{y}\right) \propto \exp \left\{-\frac{r_{1}}{2}\left(\delta-\frac{r_{2}}{r_{1}}\right)^{2}\right\}
$$

Isto é,

$$
\delta \mid \underset{\sim}{\beta, \sigma^{2}}, \underset{\sim}{y \sim N}\left\{\frac{r_{2}}{r_{1}} ; \frac{1}{r_{1}}\right\}
$$

Em resumo, as distribuições condicionais necessárias para o algoritmo Gibbs Sampling são dadas por:

$$
\begin{aligned}
& \underset{\sim}{\sigma^{2}} \underset{\sim}{\beta, \delta, y \sim I G}\left[\frac{n}{2}+c ; d+\frac{I}{2} \sum_{i=1}^{n}\left(y_{i}-x_{1 i} \beta_{1}-x_{2 i} \beta_{2}-x_{3 i} \beta_{3}-w_{i} \delta\right)^{2}\right] \\
& \beta_{1} \mid \beta_{(1)}, \delta, \sigma^{2}, y \sim N\left\{\frac{k_{12}}{k_{11}} ; \frac{l}{k_{11}}\right\} \\
& \beta_{2} \mid \beta_{(2)}, \delta, \sigma^{2}, y \sim N\left\{\frac{k_{22}}{k_{21}} ; \frac{1}{k_{21}}\right\} ; \\
& \beta_{3} \mid \beta_{(3)}, \delta, \sigma^{2}, \underset{\sim}{y} \sim N\left\{\frac{k_{32}}{k_{31}} ; \frac{l}{k_{31}}\right\} \\
& \delta \mid \underset{\sim}{\beta, \sigma^{2}}, \underset{\sim}{y \sim N}\left\{\frac{r_{2}}{r_{1}} ; \frac{1}{r_{1}}\right\} ;
\end{aligned}
$$


onde,

$$
\begin{aligned}
& k_{j 1}=\frac{1}{b^{2}}+\frac{b_{i j}}{\sigma^{2}} ; j=1,2,3 ; \\
& k_{12}=\frac{a_{1}}{\sigma^{2}}-\frac{b_{12} \beta_{2}}{\sigma^{2}}-\frac{b_{31} \beta_{3}}{\sigma^{2}}-\frac{c_{1} \delta}{\sigma^{2}} ; \\
& k_{22}=\frac{a_{2}}{\sigma^{2}}-\frac{b_{12} \beta_{1}}{\sigma^{2}}-\frac{b_{23} \beta_{3}}{\sigma^{2}}-\frac{c_{2} \delta}{\sigma^{2}} ; \\
& k_{32}=\frac{a_{3}}{\sigma^{2}}-\frac{b_{31} \beta_{1}}{\sigma^{2}}-\frac{b_{23} \beta_{2}}{\sigma^{2}}-\frac{c_{3} \delta}{\sigma^{2}} ; \\
& r_{1}=\frac{1}{a^{2}}+\frac{e}{\sigma^{2}} ; \\
& r_{2}=\frac{f}{\sigma^{2}}-\frac{c_{1} \beta_{1}}{\sigma^{2}}-\frac{c_{2} \beta_{2}}{\sigma^{2}}-\frac{c_{3} \beta_{3}}{\sigma^{2}} ;
\end{aligned}
$$

Com os dados da Tabela 10 temos os resultados para $a_{j}=\sum_{i=l}^{n} y_{i} x_{j i}, j=1,2,3$; $b_{j k}=\sum_{i=1}^{n} x_{j i} x_{b}, j, k=1,2,3 ; c_{j}=\sum_{i=1}^{n} x_{j i} w_{i}, j=1,2,3 ; f=\sum_{i=1}^{n} y_{i} w_{i}$ e $e=\sum_{i=1}^{n} w_{i}^{2}$, dados por,

$a_{1}=4.387 ; \quad a_{2}=6.435 ; \quad a_{3}=2.411 ; \quad b_{11}=1.667 ; \quad b_{12}=b_{21}=2.319 ; \quad b_{13}=b_{31}=0.815 ; \quad b_{22}=3.568 ;$ $b_{23}=b_{32}=1.255 ; b_{33}=0.483 ; c_{1}=1.04 ; c_{2}=1.83 ; c_{3}=0.85 ; f=2.78 ; e=3.0$.

Para as observações perdidas nos vetores de covariáveis $x_{i}, i=1,2,3$, geramos valores em cada iteração do algoritmo Gibbs Sampling a partir da distribuição $N\left(\bar{x}_{i}, S_{i}^{2}\right)$ onde $\bar{x}_{i}$ é a média amostral das observações completas em $x_{i}$ e $S_{1}^{2}$ é a variância amostral.

Considerando o modelo (6.2) para os dados da Tabela 10, e as distribuições a priori (6.7) para os parâmetros do modelo, com $a=2, b=2, c=2.5, d=2$, geramos cinco cadeias de Gibbs cada uma com 2000 iterações, sendo as 400 primeiras observações eliminadas ("burn-in samples"). A convergência das amostras de Gibbs foram monitoradas usando o método de Gelman e Rubin 
(1992) que usa a técnica de análise de variância para determinar se iterações adicionais são necessárias. Para cada parâmetro consideramos uma iteração em cada 10.

\subsubsection{Resultados}

Na Tabela 11 temos os sumários a posteriori e os valores $\sqrt{\hat{R}}$ (critério de Gelman \& Rubin). Para todos os parâmetros observamos a convergência do algoritmo Gibbs Sampling ( $\sqrt{\hat{R}}<1.1$ para todos os parâmetros).

Observamos que as covariáveis $x_{1}$ (duração do tratamento); $x_{2}$ (tipo de hormônio) e $x_{3}$ (obesidade) não indicam efeitos significativos no risco relativo (observar que os intervalos de credibilidade incluem zero). Também observamos que não há uma diferença significativa entre os possiveis grupos: estudos com dados perdidos e estudos com dados completos para as covariáveis, pois o intervalo de credibilidade para $\delta$ também inclui zero.

Tabela 11: Sumários a posteriori ( $x_{1}$ é o vetor duração maior ou igual à 60 meses)

\begin{tabular}{cccc}
\hline Parâmetros & Média a posteriori & $\begin{array}{c}\text { Intervalo de } \\
\text { Credibilidade (95\%) }\end{array}$ & $\sqrt{\hat{\mathbf{R}}}$ \\
\hline$\beta_{1}$ & 0.8487 & $(-1.7650 ; 3.3098)$ & 1.0079 \\
$\beta_{2}$ & 0.9030 & $(-1.1760 ; 2.8863)$ & 1.0076 \\
$\beta_{3}$ & 1.2799 & $(-2.3440 ; 4.6683)$ & 0.9987 \\
$\delta$ & -2.5403 & $(-1.4336 ; 1.0459)$ & 0.9988 \\
$\sigma^{2}$ & 0.6881 & $(2.4733 ; 1.6809)$ & 1.0007 \\
\hline
\end{tabular}

Nas Figuras 10 e 11 temos as representações gráficas dos sumários a posteriori: os gráficos das distribuições a posteriori marginais aproximadas pelas amostras geradas pelo algoritmo de Gibbs e gráficos de convergência das amostras geradas. 

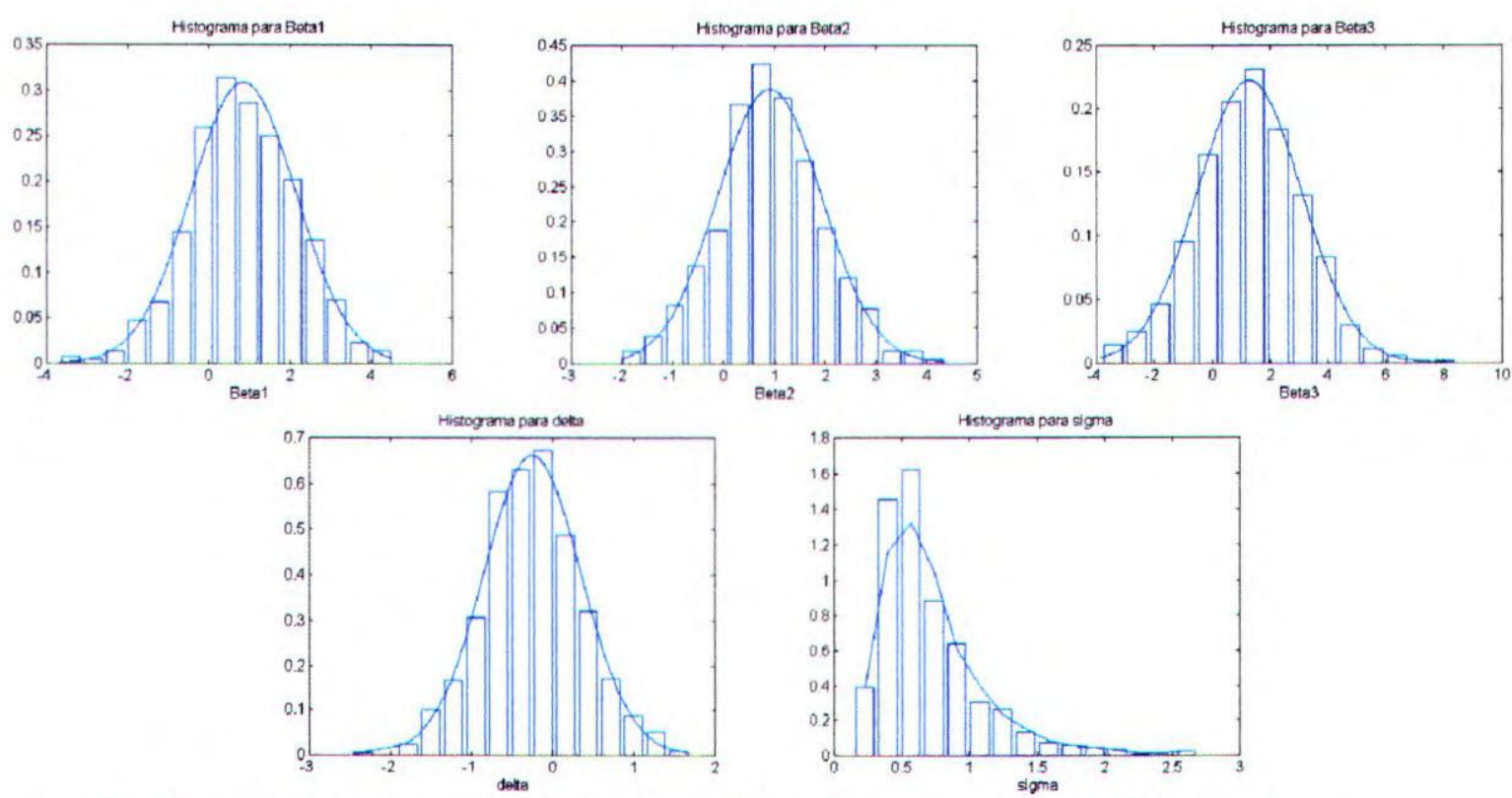

Figura 10: Densidades a posteriori marginais (duração maior do que 60 meses)
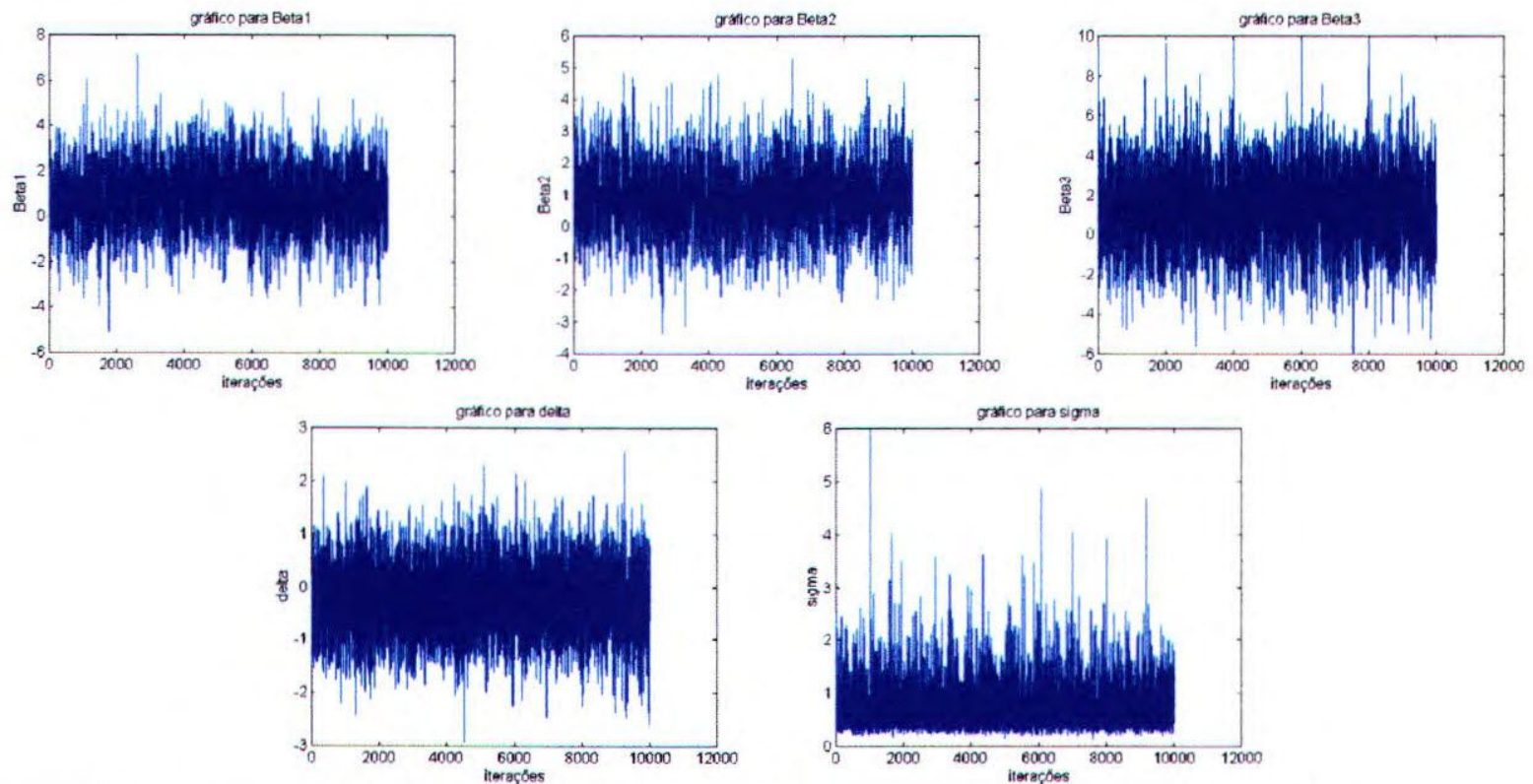

Figura 11: Convergência das amostras geradas (duração maior do que 60 meses)

Também poderíamos considerar o vetor de covariáveis $x_{1}$ como a proporção de usuárias de estrógeno por pelo menos 36 meses em lugar dos 60 meses considerados previamente. Essa proporção pode ser medida pelo número médio das usuárias de estrógeno no intervalo de 12 a 60 meses adicionado das usuárias de estrógeno por pelo menos 60 meses. Dessa forma, a primeira coluna da matriz $X$ dada em (6.5) é substituída por, $x_{1}^{\prime}=(0.63,0.19,0.65,0.63,0.75,0.45,0.69$, 
0.68). Na Tabela 12 temos os sumários a posteriori e os valores de $\sqrt{\hat{R}}$ considerando $x_{1}$ a proporção de usuárias de estrógeno por pelo menos 36 meses.

Tabela 12: Sumários a posteriori ( $x$, é o vetor de duração maior ou igual à 36 meses)

\begin{tabular}{cccc}
\hline Parâmetros & Média a posteriori & $\begin{array}{c}\text { Intervalo de } \\
\text { Credibilidade (95\%) }\end{array}$ & $\sqrt{\hat{\boldsymbol{R}}}$ \\
\hline$\beta_{1}$ & 0.9420 & $(-1.2622 ; 3.2106)$ & 1.0031 \\
$\beta_{2}$ & 0.6367 & $(-1.6514 ; 2.9367)$ & 1.0000 \\
$\beta_{3}$ & 1.2541 & $(-2.5251 ; 4.8782)$ & 1.0004 \\
$\delta$ & -2.5060 & $(-1.4308 ; 1.0045)$ & 1.0015 \\
$\sigma^{2}$ & 0.6990 & $(0.2873 ; 0.1593)$ & 1.0057 \\
\hline
\end{tabular}

Nas Figuras 12 e 13, temos as representações gráficas dos sumários a posteriori.
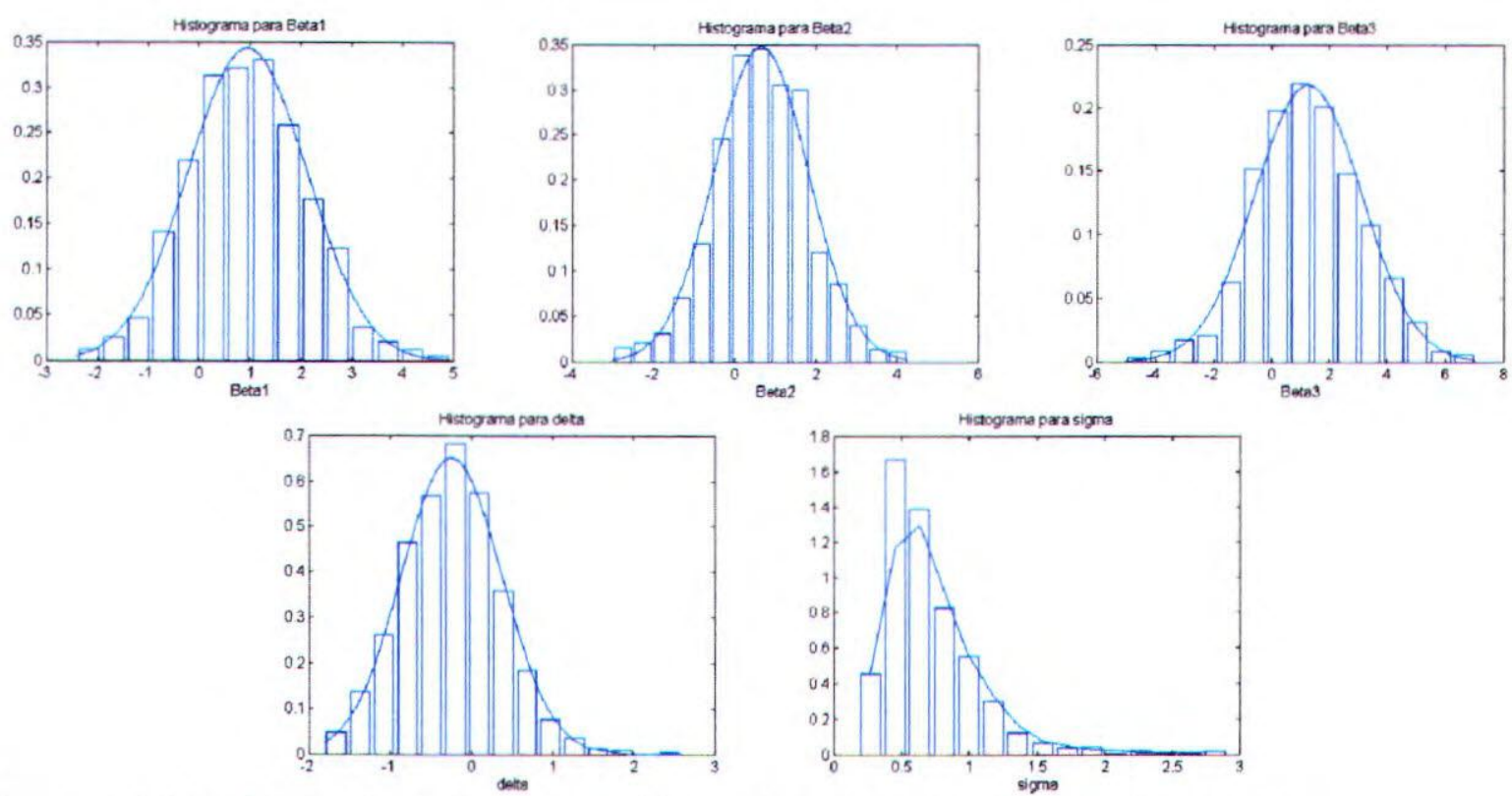

Figura 12: Densidades a posteriori marginais (duração maior do que 36 meses) 

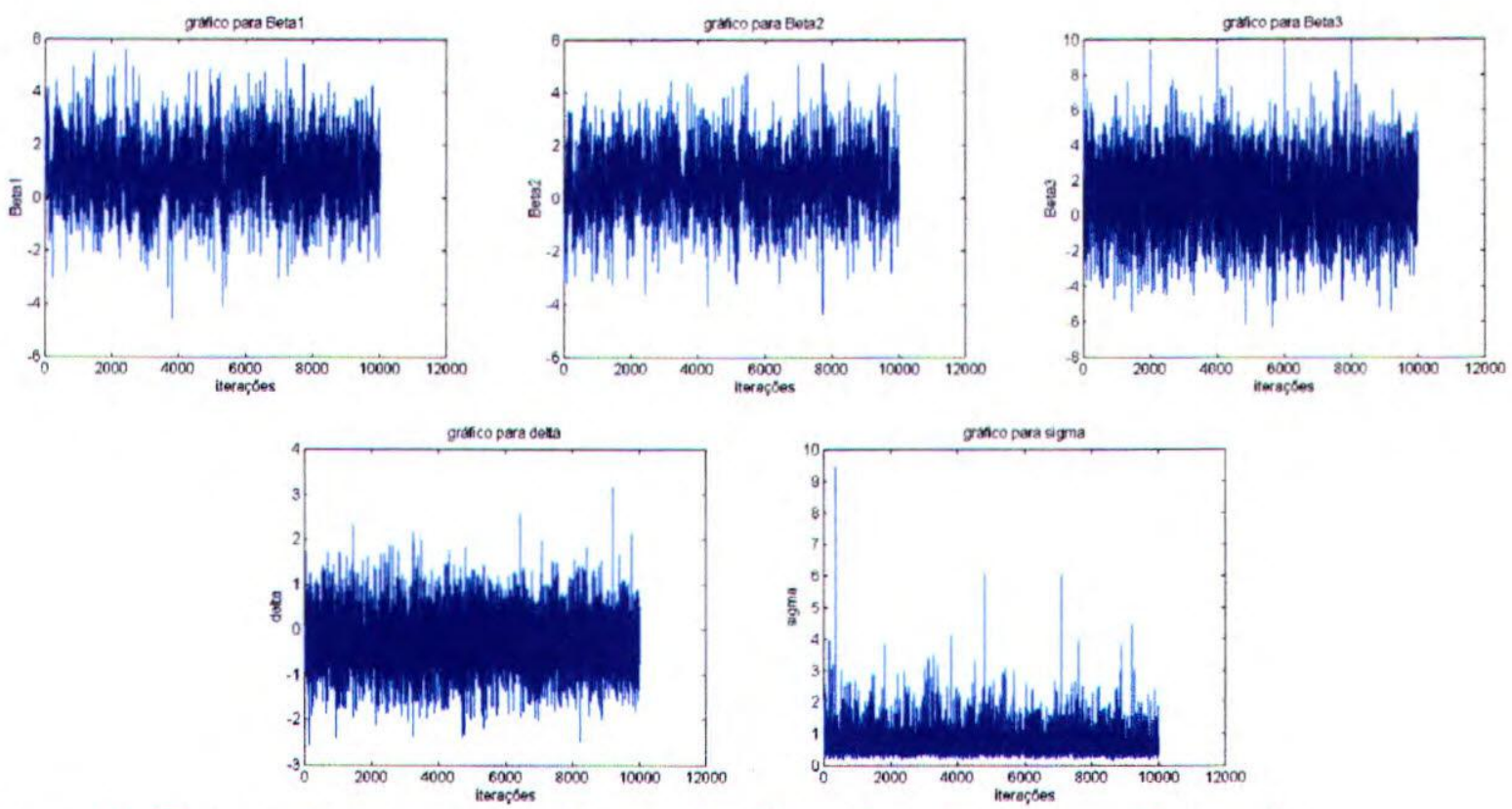

Figura 13: Convergência das amostras geradas (duração maior do que 36 meses)

A partir dos resultados obtidos na Tabela 12, observamos uma concordância com os resultados obtidos na Tabela 11, isto é, ainda indicando um efeito positivo da duração do tempo usando estrógeno (36 meses), tipo de estrógeno e obesidade, mas não significativos.

\subsection{Relação entre o uso de estrógeno e a incidência de casos de câncer nos seios}

Na Tabela 13, temos um conjunto de dados introduzidos por Larose e Dey (1995 b) envolvendo vários estudos sobre a relação da incidência de câncer nos seios e o uso de hormônios .

Para analisar os dados da Tabela 13, assumir o modelo (6.2) onde $y$ é o vetor das respostas $y=\log (R R), R R$ denota o risco relativo de uma mulher ter câncer nos seios entre as

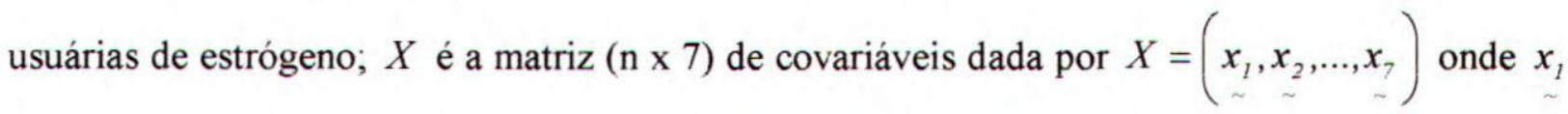
é o vetor de covariáveis representando a proporção de mulheres que tiveram pelo menos três filhos; $x_{2}$ denota a proporção de mulheres que tiveram o primeiro filho com pelo menos 30 anos; $x_{3}$ denota a proporção de mulheres que tiveram a primeira menstruação com pelo menos 
15 anos; $x_{4}$ denota a proporção de mulheres com tipo de menopausa cirúrgica; $x_{5}$ denota a proporção de mulheres com história de casos de câncer na família; $x_{6}$ denota a proporção de mulheres que usaram estrógeno por pelo menos 10 anos e $x_{7}$ denota a proporção de mulheres que entraram na menopausa com pelo menos 50 anos. Também consideramos o vetor $(\mathrm{n} \times 1) w$ onde $w_{i}=I(0) ; i=1,2, \ldots, n$ indica informação completa (ou não) para as covariáveis.

Tabela 13: Dados de laboratórios diferentes (incidência de câncer nos seios)

\begin{tabular}{|c|c|c|c|c|c|c|c|c|c|c|}
\hline $\begin{array}{c}\text { Labora } \\
\text {-tórios }\end{array}$ & Resposta & \multicolumn{4}{|c|}{$\mathbf{N}^{\mathbf{0}}$ de Filhos } & \multicolumn{3}{c|}{$\begin{array}{c}\text { Idade no Primeiro } \\
\text { Parto }\end{array}$} & \multicolumn{2}{|c|}{$\begin{array}{c}\text { Idade na Primeira } \\
\text { Menstruação }\end{array}$} \\
\hline & $\log (\boldsymbol{R R})$ & $\mathbf{0}$ & $\mathbf{1 - 2}$ & $\geq \mathbf{3}$ & $<\mathbf{2 0}$ & $\mathbf{2 0 - 2 9}$ & $\geq \mathbf{3 0}$ & $<\mathbf{1 2}$ & $\mathbf{1 2 - 1 4}$ & $\geq \mathbf{1 5}$ \\
\hline $\mathbf{1}$ & 0.0245 & 345 & 683 & 539 & 155 & 848 & 219 & 287 & 1031 & 242 \\
\hline $\mathbf{2}$ & 0.0746 & 576 & 1266 & 1230 & 387 & 2556 & 376 & 590 & 2098 & 408 \\
\hline $\mathbf{3}$ & 0.0827 & 204 & 731 & 770 & 113 & 1116 & 271 & 294 & 1194 & 201 \\
\hline $\mathbf{4}$ & -0.0419 & 331 & 981 & 1678 & 721 & 1740 & 136 & 666 & 1987 & 352 \\
\hline $\mathbf{5}$ & 0.0185 & 359 & 733 & 844 & 146 & 1120 & 311 & 317 & 1361 & 250 \\
\hline $\mathbf{6}$ & 0.0172 & 210 & 440 & 422 & 81 & 613 & 168 & 194 & 691 & 181 \\
\hline $\mathbf{7}$ & 0.1793 & - & - & - & - & - & - & 202 & 717 & 403 \\
\hline $\mathbf{8}$ & 0.1487 & 112 & 298 & 340 & 53 & 433 & 121 & 172 & 467 & 109 \\
\hline $\mathbf{9}$ & 0.3338 & 197 & 411 & 473 & - & - & - & - & - & - \\
\hline
\end{tabular}

\begin{tabular}{|c|c|c|c|c|c|c|c|c|c|c|}
\hline $\begin{array}{c}\text { Labora } \\
\text {-tórios }\end{array}$ & \multicolumn{3}{|c|}{$\begin{array}{c}\text { Tipo de } \\
\text { Menopausa }\end{array}$} & $\begin{array}{c}\text { História de Câncer } \\
\text { nos Seios na } \\
\text { Família }\end{array}$ & \multicolumn{2}{c|}{$\begin{array}{c}\text { Duração do Trata- } \\
\text { mento com Estró- } \\
\text { geno (em anos) }\end{array}$} & \multicolumn{3}{|c|}{$\begin{array}{c}\text { Idade no Início da } \\
\text { Menopausa }\end{array}$} \\
\hline & Natural & Cirúrg. & Positivo & Negativo & $\leq \mathbf{1}$ & $\mathbf{1 - 9}$ & $\geq \mathbf{1 0}$ & $<\mathbf{4 0}$ & $\mathbf{4 0 - 4 9}$ & $\geq \mathbf{5 0}$ \\
\hline $\mathbf{1}$ & 600 & 244 & 130 & 1438 & 49 & 108 & 45 & 42 & 243 & 370 \\
\hline $\mathbf{2}$ & 2134 & 961 & 335 & 2761 & 187 & 259 & 105 & 571 & 1156 & 1239 \\
\hline $\mathbf{3}$ & 1274 & 432 & 244 & 1416 & 177 & 142 & 29 & 164 & 695 & 808 \\
\hline $\mathbf{4}$ & 1913 & 1101 & 279 & 1639 & 186 & 632 & 137 & - & - & - \\
\hline $\mathbf{5}$ & 2435 & 1002 & 498 & 1440 & 589 & 1025 & 536 & 304 & 879 & 761 \\
\hline $\mathbf{6}$ & - & - & 85 & 982 & 115 & 308 & 89 & 168 & 378 & 378 \\
\hline $\mathbf{7}$ & 942 & 380 & - & - & 100 & 211 & 177 & 126 & 629 & 557 \\
\hline $\mathbf{8}$ & - & - & 92 & 656 & - & - & - & 96 & 276 & 376 \\
\hline $\mathbf{9}$ & 1081 & 201 & 70 & 1011 & 63 & 103 & 41 & - & - & - \\
\hline
\end{tabular}


Assim a matriz $X$ é dada por

$$
X=\left(\begin{array}{ccccccc}
0.34 & 0.18 & 0.15 & 0.29 & 0.08 & 0.22 & 0.56 \\
0.40 & 0.11 & 0.13 & 0.31 & 0.11 & 0.19 & 0.42 \\
0.45 & 0.18 & 0.12 & 0.25 & 0.15 & 0.08 & 0.48 \\
0.56 & 0.05 & 0.12 & 0.36 & 0.14 & 0.14 & - \\
0.44 & 0.20 & 0.13 & 0.29 & 0.26 & 0.25 & 0.39 \\
0.39 & 0.19 & 0.17 & - & 0.08 & 0.17 & 0.41 \\
- & - & 0.30 & 0.29 & - & 0.36 & 0.42 \\
0.45 & 0.20 & 0.15 & - & 0.12 & - & 0.50 \\
0.44 & - & - & 0.16 & 0.06 & 0.20 & -
\end{array}\right)
$$

\subsection{Análise Bayesiana para os dados da Tabela 13}

Com as mesmas suposições consideradas anteriormente e com as mesmas distribuições a priori para os parâmetros dadas em (6.7), onde $\beta_{m}, m=1,2, \ldots, 7$ são os parâmetros de regressão associados com as 7 covariáveis, a função de verossimilhança e a distribuição a priori conjunta para os parâmetros são definidas como segue.

\subsubsection{Função de verossimilhança}

A função de verossimilhança para os parâmetros $\beta, \delta$ e $\sigma^{2}$ é dada por,

$$
\begin{aligned}
L\left(\beta, \delta, \sigma^{2}\right)= & \coprod_{i=1}^{n} \frac{1}{\sqrt{2 \pi \sigma^{2}}} \exp \left\{-\frac{1}{2 \sigma^{2}}\left(y-(X \underset{\sim}{\sim}-W \delta)^{2}\right)\right\} \\
\propto & \left(\sigma^{2}\right)^{-n / 2} \exp \left\{-\frac{1}{2 \sigma^{2}} \sum_{i=1}^{n}\left(y_{i}-x_{1 i} \beta_{1}-x_{2 i} \beta_{2}-x_{3 i} \beta_{3}-x_{4 i} \beta_{4}-x_{5 i} \beta_{5}-x_{6 i} \beta_{6}-\right.\right. \\
& \left.\left.-x_{7 i} \beta_{7}-w_{i} \delta\right)^{2}\right\}
\end{aligned}
$$




\subsubsection{Distribuição a priori}

Considerando as mesmas distribuições a priori dadas em (6.7), a distribuição a priori conjunta é dada por,

$$
\begin{aligned}
\pi\left(\beta, \delta, \sigma^{2}\right) \propto & \exp \left\{-\frac{1}{2 a^{2}}\left(\delta^{2}\right)\right\} \exp \left\{-\frac{1}{2 b^{2}}\left(\beta_{1}^{2}+\beta_{2}^{2}+\beta_{3}^{2}+\beta_{4}^{2}+\beta_{5}^{2}+\beta_{6}^{2}+\beta_{7}^{2}\right)\right\} \times \\
& \times\left(\sigma^{2}\right)^{-(c+1)} \exp \left\{-\frac{d}{\sigma^{2}}\right\}
\end{aligned}
$$

\subsubsection{Distribuição a posteriori conjunta}

A distribuição a posteriori conjunta para $\beta, \delta$ e $\sigma^{2}$, obtida a partir de (6.16) e (6.17) é dada por,

$$
\begin{aligned}
& \pi\left(\beta, \delta, \sigma^{2} \mid \underset{\sim}{y}\right) \propto \exp \left\{-\frac{1}{2 a^{2}}\left(\delta^{2}\right)\right\} \exp \left\{-\frac{1}{2 b^{2}}\left(\beta_{1}^{2}+\beta_{2}^{2}+\beta_{3}^{2}+\beta_{4}^{2}+\beta_{s}^{2}+\beta_{6}^{2}+\beta_{7}^{2}\right)\right\} \times \\
& \times\left(\sigma^{2}\right)^{-(n / 2+c+1)} \exp \left\{-\frac{1}{\sigma^{2}}\left[d+\frac{1}{2} \sum_{i=1}^{n}\left(y_{i}-x_{1 i} \beta_{1}-x_{2 i} \beta_{2}-x_{3 i} \beta_{3}-x_{4 i} \beta_{4}-x_{5 i} \beta_{5}-\right.\right.\right. \\
& \left.\left.\left.-x_{6 i} \beta_{6}-x_{7 i} \beta_{7}-w_{i} \delta\right)^{2}\right]\right\}
\end{aligned}
$$

$\mathrm{Na}$ forma matricial, usando o resultado (6.10), a distribuição a posteriori conjunta pode ser rescrita da mesma forma que (6.11), ou seja,

$$
\begin{aligned}
& \left.\pi\left(\underset{\sim}{\beta}, \delta, \sigma^{2} \mid \underset{\sim}{y}\right) \propto\left(\sigma^{2}\right)^{-\left(\frac{n}{2}+c+1\right.}\right) \exp \left\{-\frac{1}{2 a^{2}} \delta^{\prime} \delta-\frac{1}{2 b^{2}} \beta_{\sim}^{\prime} \underset{\sim}{\beta}\right\} \times \exp \left\{-\frac{1}{\sigma^{2}}\left[d+\frac{1}{2}\left(\underset{\sim}{y^{\prime}} \underset{\sim}{y-}\right.\right.\right. \\
& \left.\left.\left.-2 \underset{\sim}{y^{\prime}} X \underset{\sim}{\beta} \underset{\sim}{2} \underline{\sim}^{\prime} \underset{\sim}{w} \delta+\underline{\sim}^{\prime} X^{\prime} X \underset{\sim}{\beta}+2 \underset{\sim}{\beta} X^{\prime} \underset{\sim}{w} \delta+\delta^{\prime} \underset{\sim}{w^{\prime}} \underset{\sim}{w} \delta\right)\right]\right\}
\end{aligned}
$$




\subsubsection{Aspectos computacionais}

Com a distribuição a posteriori conjunta dada acima, as distribuições condicionais requeridas para o algoritmo Gibbs Sampling são dadas por:

$$
\begin{aligned}
& \sigma_{\sim}^{2} \underset{\sim}{\beta, \delta, y \sim I G}\left[\begin{array}{c}
\frac{n}{2}+c ; d+\frac{1}{2} \sum_{i=1}^{n}\left(y_{i}-x_{11} \beta_{1}-x_{2 i} \beta_{2}-x_{3 i} \beta_{3}-x_{41} \beta_{4}-x_{51} \beta_{5}-\right. \\
\left.-x_{6 i} \beta_{6}-x_{7 i} \beta_{7}-w_{i} \delta\right)^{2}
\end{array}\right] \\
& \pi\left(\underset{\sim}{\beta} \mid \sigma^{2}, \delta, \underset{\sim}{y}\right) \propto \exp \left\{-\frac{1}{2 b^{2}} \boldsymbol{\beta}_{\sim}^{\prime} \underset{\sim}{\beta}+\frac{1}{\sigma^{2}} y_{\sim}^{\prime} X \underset{\sim}{\beta}-\frac{1}{2 \sigma^{2}} \beta_{\sim}^{\prime} X^{\prime} X \underset{\sim}{\beta}-\frac{1}{\sigma^{2}} \beta^{\prime} X^{\prime} w \delta\right\} \\
& \pi\left(\delta \mid \sigma^{2}, \underset{\sim}{\beta, y}\right) \propto \exp \left\{-\frac{1}{2 a^{2}} \delta^{\prime} \delta+\frac{1}{\sigma^{2}}{\underset{\sim}{\prime}}_{\sim}^{w} \delta-\frac{1}{2 \sigma^{2}} \delta^{\prime} \underset{\sim}{w^{\prime}} \underset{\sim}{w} \delta-\frac{1}{\sigma^{2}} \beta_{\sim}^{\prime} X^{\prime} \underset{\sim}{w} \delta\right\}
\end{aligned}
$$

Usando uma generalização dos resultados dados em (6.12) e (6.13) para as sete covariáveis consideradas nesta análise, temos

(1) $y^{\prime} X \underset{\beta}{\beta}=a_{1} \beta_{1}+a_{2} \beta_{2}+a_{3} \beta_{3}+a_{4} \beta_{4}+a_{5} \beta_{5}+a_{6} \beta_{6}+a_{7} \beta_{7}$

onde $a_{j}=\sum_{i=1}^{n} y_{i} x_{j i}, j=1,2, \ldots, 7$

(2) $\beta^{\prime} X^{\prime} X \underset{\sim}{\beta}=b_{11} \beta_{1}^{2}+2 b_{12} \beta_{1} \beta_{2}+2 b_{13} \beta_{1} \beta_{3}+2 b_{14} \beta_{1} \beta_{4}+2 b_{15} \beta_{1} \beta_{5}+2 b_{16} \beta_{1} \beta_{6}+2 b_{17} \beta_{1} \beta_{7}+$

$$
\begin{aligned}
& +b_{22} \beta_{2}^{2}+2 b_{23} \beta_{2} \beta_{3}+2 b_{24} \beta_{2} \beta_{4}+2 b_{25} \beta_{2} \beta_{5}+2 b_{26} \beta_{2} \beta_{6}+2 b_{27} \beta_{2} \beta_{7}+b_{33} \beta_{3}^{2}+ \\
& +2 b_{34} \beta_{3} \beta_{4}+2 b_{35} \beta_{3} \beta_{5}+2 b_{36} \beta_{3} \beta_{6}+2 b_{37} \beta_{3} \beta_{7}+b_{44} \beta_{4}^{2}+2 b_{45} \beta_{4} \beta_{5}+ \\
& +2 b_{46} \beta_{4} \beta_{6}+2 b_{47} \beta_{4} \beta_{7}+b_{55} \beta_{5}^{2}+2 b_{56} \beta_{5} \beta_{6}+2 b_{57} \beta_{5} \beta_{7}+b_{66} \beta_{6}^{2}+2 b_{67} \beta_{6} \beta_{7}+ \\
& +b_{77} \beta_{7}^{2}
\end{aligned}
$$

onde $b_{j k}=\sum_{i=1}^{n} x_{\mu} x_{k}, j, k=1,2, \ldots, 7$

(3) $\beta^{\prime} X^{\prime} \underset{\sim}{w} \delta=c_{1} \beta_{1} \delta+c_{2} \beta_{2} \delta+c_{3} \beta_{3} \delta+c_{4} \beta_{4} \delta+c_{5} \beta_{5} \delta+c_{6} \beta_{6} \delta+c_{7} \beta_{7} \delta$

onde $c_{j}=\sum_{i=1}^{n} x_{j i} w_{i}, j=1,2, \ldots, 7$;

(4) $\delta^{\prime} \delta=\delta^{2}$ 
(5) $y^{\prime} \underset{w}{\delta}=f \delta$

onde $f=\sum_{i=1}^{n} y_{i} w_{i}$

(6) $\delta^{\prime} \underset{w}{w} \delta=e \delta^{2}$

onde $e=\sum_{i=1}^{n} w_{i}^{2}$

Com os resuitados dados em (6.20), podemos rescrever $\pi\left(\underset{\sim}{\beta} \mid \sigma^{2}, \delta, \underset{\sim}{\mathcal{y}}\right)$ e $\pi\left(\underset{\sim}{\delta} \underset{\sim}{\beta, \sigma^{2}}, \underset{\sim}{y}\right)$ respectivamente por,

$$
\begin{aligned}
\pi\left(\beta \mid \sigma^{2}, \delta, y\right) \propto & \exp \left\{-\frac{l}{2 b^{2}}\left(\beta_{1}^{2}+\beta_{2}^{2}+\beta_{3}^{2}+\beta_{4}^{2}+\beta_{5}^{2}+\beta_{6}^{2}+\beta_{7}^{2}\right)+\frac{1}{\sigma^{2}}\left(a_{1} \beta_{1}+a_{2} \beta_{2}+\right.\right. \\
& \left.+a_{3} \beta_{3}+a_{4} \beta_{4}+a_{5} \beta_{5}+a_{6} \beta_{6}+a_{7} \beta_{7}\right)-\frac{l}{2 \sigma^{2}}\left(b_{11} \beta_{1}^{2}+2 b_{12} \beta_{1} \beta_{2}+2 b_{13} \beta_{1} \beta_{3}+\right. \\
& +2 b_{14} \beta_{1} \beta_{4}+2 b_{15} \beta_{1} \beta_{5}+2 b_{16} \beta_{1} \beta_{6}+2 b_{17} \beta_{1} \beta_{7}+b_{23} \beta_{2}^{2}+2 b_{23} \beta_{2} \beta_{3}+ \\
& +2 b_{24} \beta_{2} \beta_{4}+2 b_{25} \beta_{2} \beta_{s}+2 b_{26} \beta_{2} \beta_{6}+2 b_{27} \beta_{2} \beta_{7}+b_{33} \beta_{3}^{2}+2 b_{34} \beta_{3} \beta_{4}+ \\
& +2 b_{35} \beta_{3} \beta_{5}+2 b_{36} \beta_{3} \beta_{6}+2 b_{37} \beta_{3} \beta_{7}+b_{44} \beta_{4}^{2}+2 b_{45} \beta_{4} \beta_{5}+2 b_{46} \beta_{4} \beta_{6}+ \\
& \left.+2 b_{47} \beta_{4} \beta_{7}+b_{s 5} \beta_{5}^{2}+2 b_{56} \beta_{5} \beta_{6}+2 b_{57} \beta_{5} \beta_{7}+b_{66} \beta_{6}^{2}+2 b_{67} \beta_{6} \beta_{7}+b_{77} \beta_{7}^{2}\right)- \\
& \left.-\frac{1}{\sigma^{2}}\left(c_{1} \beta_{1} \delta+c_{2} \beta_{2} \delta+c_{3} \beta_{3} \delta+c_{4} \beta_{4} \delta+c_{5} \beta_{5} \delta+c_{6} \beta_{6} \delta+c_{7} \beta_{7} \delta\right)\right\}
\end{aligned}
$$

e

$$
\begin{aligned}
\pi\left(\delta \mid \beta, \sigma^{2}, y\right) \propto & \exp \left\{-\frac{1}{2 a^{2}}\left(\delta^{2}\right)+\frac{1}{\sigma^{2}}(f \delta)-\frac{1}{\sigma^{2}}\left(c_{1} \beta_{1} \delta+c_{2} \beta_{2} \delta+c_{3} \beta_{3} \delta+c_{4} \beta_{4} \delta+c_{5} \beta_{5} \delta+\right.\right. \\
& \left.\left.+c_{6} \beta_{6} \delta+c_{7} \beta_{7} \delta\right)-\frac{1}{2 \sigma^{2}}\left(e \delta^{2}\right)\right\}
\end{aligned}
$$

Individualmente, as distribuições condicionais para $\beta_{1}, \beta_{2}, \beta_{2}, \beta_{4}, \beta_{5}, \beta_{6}, \beta_{7}$ e $\delta$ são dadas respectivamente por: 


$$
\begin{aligned}
\pi\left(\beta_{1} \mid \beta_{(1)}, \delta, \sigma^{2}, \underset{\sim}{y}\right) \propto & \exp \left\{-\frac{I}{2 b^{2}} \beta_{l}^{2}+\frac{a_{l} \beta_{l}}{\sigma^{2}}-\frac{1}{2 \sigma^{2}}\left(b_{11} \beta_{l}^{2}+2 b_{12} \beta_{1} \beta_{2}+2 b_{13} \beta_{l} \beta_{3}+\right.\right. \\
& \left.\left.+2 b_{14} \beta_{l} \beta_{4}+2 b_{15} \beta_{1} \beta_{5}+2 b_{16} \beta_{l} \beta_{6}+2 b_{17} \beta_{1} \beta_{7}\right)-\frac{1}{\sigma^{2}}\left(c_{1} \beta_{l} \delta\right)\right\}
\end{aligned}
$$

$$
\begin{aligned}
\pi\left(\beta_{2} \mid \beta_{(2)}, \delta, \sigma^{2}, \underset{\sim}{y}\right) \propto & \exp \left\{-\frac{1}{2 b^{2}} \beta_{2}^{2}+\frac{a_{2} \beta_{2}}{\sigma^{2}}-\frac{1}{2 \sigma^{2}}\left(b_{22} \beta_{2}^{2}+2 b_{12} \beta_{1} \beta_{2}+2 b_{23} \beta_{2} \beta_{3}+\right.\right. \\
& \left.\left.+2 b_{24} \beta_{2} \beta_{4}+2 b_{25} \beta_{2} \beta_{5}+2 b_{26} \beta_{2} \beta_{6}+2 b_{27} \beta_{2} \beta_{7}\right)-\frac{1}{\sigma^{2}}\left(c_{2} \beta_{2} \delta\right)\right\}
\end{aligned}
$$

$\pi\left(\beta_{3} \mid \beta_{(3)}, \delta, \sigma^{2}, \underset{\sim}{y}\right) \propto \exp \left\{-\frac{1}{2 b^{2}} \beta_{3}^{2}+\frac{a_{3} \beta_{3}}{\sigma^{2}}-\frac{1}{2 \sigma^{2}}\left(b_{33} \beta_{3}^{2}+2 b_{31} \beta_{3} \beta_{1}+2 b_{32} \beta_{3} \beta_{2}+\right.\right.$

$$
\left.\left.+2 b_{34} \beta_{3} \beta_{4}+2 b_{35} \beta_{3} \beta_{5}+2 b_{36} \beta_{3} \beta_{6}+2 b_{37} \beta_{3} \beta_{7}\right)-\frac{1}{\sigma^{2}}\left(c_{3} \beta_{3} \delta\right)\right\}
$$

$\pi\left(\beta_{4} \mid \beta_{\sim}^{\left.\beta_{4}\right)}, \delta, \sigma^{2}, \underset{\sim}{y}\right) \propto \exp \left\{-\frac{I}{2 b^{2}} \beta_{4}^{2}+\frac{a_{4} \beta_{4}}{\sigma^{2}}-\frac{1}{2 \sigma^{2}}\left(b_{44} \beta_{4}^{2}+2 b_{41} \beta_{4} \beta_{1}+2 b_{42} \beta_{4} \beta_{2}+\right.\right.$

$$
\left.\left.+2 b_{43} \beta_{4} \beta_{3}+2 b_{45} \beta_{4} \beta_{5}+2 b_{46} \beta_{4} \beta_{6}+2 b_{47} \beta_{4} \beta_{7}\right)-\frac{1}{\sigma^{2}}\left(c_{4} \beta_{4} \delta\right)\right\}
$$

$\pi\left(\beta_{s} \mid \underset{\sim}{\beta_{(s)}}, \delta, \sigma^{2}, \underset{\sim}{y}\right) \propto \exp \left\{-\frac{1}{2 b^{2}} \beta_{s}^{2}+\frac{a_{s} \beta_{s}}{\sigma^{2}}-\frac{I}{2 \sigma^{2}}\left(b_{s s} \beta_{s}^{2}+2 b_{s 1} \beta_{s} \beta_{1}+2 b_{s 2} \beta_{s} \beta_{2}+\right.\right.$

$$
\left.\left.+2 b_{53} \beta_{s} \beta_{3}+2 b_{54} \beta_{s} \beta_{4}+2 b_{s 6} \beta_{s} \beta_{6}+2 b_{s 7} \beta_{s} \beta_{7}\right)-\frac{l}{\sigma^{2}}\left(c_{5} \beta_{5} \delta\right)\right\}
$$

$$
\begin{aligned}
\pi\left(\beta_{6} \mid \beta_{(\sigma)}, \delta, \sigma^{2}, \underset{\sim}{y}\right) \propto & \exp \left\{-\frac{1}{2 b^{2}} \beta_{6}^{2}+\frac{a_{6} \beta_{6}}{\sigma^{2}}-\frac{l}{2 \sigma^{2}}\left(b_{66} \beta_{6}^{2}+2 b_{61} \beta_{6} \beta_{1}+2 b_{62} \beta_{6} \beta_{2}+\right.\right. \\
& \left.\left.+2 b_{63} \beta_{6} \beta_{3}+2 b_{64} \beta_{6} \beta_{4}+2 b_{65} \beta_{6} \beta_{5}+2 b_{67} \beta_{6} \beta_{7}\right)-\frac{1}{\sigma^{2}}\left(c_{6} \beta_{6} \delta\right)\right\}
\end{aligned}
$$

$$
\begin{array}{r}
\pi\left(\beta_{7} \mid \beta_{(7)}, \delta, \sigma^{2}, \underset{\sim}{y}\right) \propto \exp \left\{-\frac{1}{2 b^{2}} \beta_{7}^{2}+\frac{a_{7} \beta_{7}}{\sigma^{2}}-\frac{l}{2 \sigma^{2}}\left(b_{77} \beta_{7}^{2}+2 b_{71} \beta_{7} \beta_{1}+2 b_{72} \beta_{7} \beta_{2}+\right.\right. \\
\left.\left.+2 b_{73} \beta_{7} \beta_{3}+2 b_{74} \beta_{7} \beta_{4}+2 b_{75} \beta_{7} \beta_{5}+2 b_{76} \beta_{7} \beta_{6}\right)-\frac{l}{\sigma^{2}}\left(c_{7} \beta_{7} \delta\right)\right\}
\end{array}
$$

$$
\beta_{(i)}=\left(\beta_{1}, \ldots, \beta_{i-1}, \beta_{i+1}, \ldots, \beta_{7}\right) ; i=1,2, \ldots, 7
$$




$$
\begin{aligned}
\pi\left(\delta \mid \beta, \sigma^{2}, y\right) \propto & \exp \left\{-\frac{1}{2 a^{2}}\left(\delta^{2}\right)+\frac{1}{\sigma^{2}}(f \delta)-\frac{1}{\sigma^{2}}\left(c_{1} \beta_{1} \delta+c_{2} \beta_{2} \delta+c_{3} \beta_{3} \delta+c_{4} \beta_{4} \delta+\right.\right. \\
& \left.\left.+c_{5} \beta_{5} \delta+c_{6} \beta_{6} \delta+c_{7} \beta_{7} \delta\right)-\frac{1}{2 \sigma^{2}}\left(e \delta^{2}\right)\right\}
\end{aligned}
$$

Definir os parâmetros,

$$
\begin{aligned}
& k_{j 1}=\frac{1}{b^{2}}+\frac{b_{j i}}{\sigma^{2}} ; j=1,2, \ldots, 7 ; \\
& k_{12}=\frac{a_{1}}{\sigma^{2}}-\frac{b_{12} \beta_{2}}{\sigma^{2}}-\frac{b_{13} \beta_{3}}{\sigma^{2}}-\frac{b_{14} \beta_{4}}{\sigma^{2}}-\frac{b_{15} \beta_{5}}{\sigma^{2}}-\frac{b_{16} \beta_{6}}{\sigma^{2}}-\frac{b_{17} \beta_{7}}{\sigma^{2}}-\frac{c_{1} \delta}{\sigma^{2}} \\
& k_{22}=\frac{a_{2}}{\sigma^{2}}-\frac{b_{12} \beta_{1}}{\sigma^{2}}-\frac{b_{23} \beta_{3}}{\sigma^{2}}-\frac{b_{24} \beta_{4}}{\sigma^{2}}-\frac{b_{25} \beta_{5}}{\sigma^{2}}-\frac{b_{26} \beta_{6}}{\sigma^{2}}-\frac{b_{27} \beta_{7}}{\sigma^{2}}-\frac{c_{2} \delta}{\sigma^{2}} \\
& k_{32}=\frac{a_{3}}{\sigma^{2}}-\frac{b_{13} \beta_{1}}{\sigma^{2}}-\frac{b_{23} \beta_{2}}{\sigma^{2}}-\frac{b_{34} \beta_{4}}{\sigma^{2}}-\frac{b_{35} \beta_{5}}{\sigma^{2}}-\frac{b_{36} \beta_{6}}{\sigma^{2}}-\frac{b_{37} \beta_{7}}{\sigma^{2}}-\frac{c_{3} \delta}{\sigma^{2}} \\
& k_{42}=\frac{a_{4}}{\sigma^{2}}-\frac{b_{14} \beta_{1}}{\sigma^{2}}-\frac{b_{23} \beta_{2}}{\sigma^{2}}-\frac{b_{34} \beta_{3}}{\sigma^{2}}-\frac{b_{45} \beta_{5}}{\sigma^{2}}-\frac{b_{46} \beta_{6}}{\sigma^{2}}-\frac{b_{47} \beta_{7}}{\sigma^{2}}-\frac{c_{4} \delta}{\sigma^{2}} \\
& k_{52}=\frac{a_{5}}{\sigma^{2}}-\frac{b_{15} \beta_{1}}{\sigma^{2}}-\frac{b_{25} \beta_{2}}{\sigma^{2}}-\frac{b_{35} \beta_{3}}{\sigma^{2}}-\frac{b_{45} \beta_{4}}{\sigma^{2}}-\frac{b_{56} \beta_{6}}{\sigma^{2}}-\frac{b_{57} \beta_{7}}{\sigma^{2}}-\frac{c_{5} \delta}{\sigma^{2}} \\
& k_{62}=\frac{a_{6}}{\sigma^{2}}-\frac{b_{16} \beta_{1}}{\sigma^{2}}-\frac{b_{26} \beta_{2}}{\sigma^{2}}-\frac{b_{36} \beta_{3}}{\sigma^{2}}-\frac{b_{46} \beta_{4}}{\sigma^{2}}-\frac{b_{56} \beta_{5}}{\sigma^{2}}-\frac{b_{67} \beta_{7}}{\sigma^{2}}-\frac{c_{6} \delta}{\sigma^{2}} ; \\
& k_{72}=\frac{a_{7}}{\sigma^{2}}-\frac{b_{17} \beta_{1}}{\sigma^{2}}-\frac{b_{27} \beta_{2}}{\sigma^{2}}-\frac{b_{37} \beta_{3}}{\sigma^{2}}-\frac{b_{47} \beta_{4}}{\sigma^{2}}-\frac{b_{57} \beta_{5}}{\sigma^{2}}-\frac{b_{67} \beta_{6}}{\sigma^{2}}-\frac{c_{7} \delta}{\sigma^{2}}
\end{aligned}
$$$$
r_{j l}=\frac{1}{a^{2}}+\frac{e_{i j}}{\sigma^{2}} ; j=1,2
$$$$
r_{12}=\frac{f}{\sigma^{2}}-\frac{c_{1} \beta_{1}}{\sigma^{2}}-\frac{c_{2} \beta_{2}}{\sigma^{2}}-\frac{c_{3} \beta_{3}}{\sigma^{2}}-\frac{c_{4} \beta_{4}}{\sigma^{2}}-\frac{c_{5} \beta_{5}}{\sigma^{2}}-\frac{c_{6} \beta_{6}}{\sigma^{2}}-\frac{c_{7} \beta_{7}}{\sigma^{2}}
$$

onde,

$$
a_{j}=\sum_{i=1}^{n} y_{i} x_{j i} ; j=1,2, \ldots, 7
$$




$$
\begin{aligned}
& b_{j k}=\sum_{i=1}^{n} x_{j i} x_{k j} ; j, k=1,2, \ldots, 7 ; \\
& c_{j}=\sum_{i=1}^{n} x_{j t} w_{i} ; j=1,2, \ldots, 7 \\
& f=\sum_{i=1}^{n} y_{i} w_{i} ; \\
& e=\sum_{i=1}^{n} w_{i}^{2} ; j, k=1,2 ;
\end{aligned}
$$

As distribuições condicionais necessárias para o algoritmo Gibbs Sampling para $\sigma^{2}, \beta_{1}, \beta_{2}, \beta_{3}, \beta_{4}, \beta_{5}, \beta_{6}, \beta_{7}$ e $\delta$ são dadas por:

$\sigma_{\sim}^{2} \underset{\sim}{\beta, \delta, y \sim I G}\left[\begin{array}{c}\frac{n}{2}+c ; d+\frac{1}{2} \sum_{i=i}^{n}\left(y_{i}-x_{1 i} \beta_{1}-x_{2 i} \beta_{2}-x_{3 i} \beta_{3}-x_{4 i} \beta_{4}-x_{5 i} \beta_{5}-\right. \\ \left.-x_{6 i} \beta_{6}-x_{7 i} \beta_{7}-w_{i} \delta\right)^{2}\end{array}\right]$

$$
\beta_{1} \mid \beta_{(1)}, \delta, \sigma^{2}, \underset{\sim}{y} \sim N\left\{\frac{k_{l 2}}{k_{l l}} ; \frac{1}{k_{l l}}\right\}
$$$$
\beta_{2} \mid \beta_{(2)}, \delta, \sigma^{2}, \underset{\sim}{y} \sim N\left\{\frac{k_{22}}{k_{21}} ; \frac{1}{k_{21}}\right\} ;
$$$$
\beta_{3} \mid \beta_{(3)}, \delta, \sigma^{2}, y \sim N\left\{\frac{k_{32}}{k_{31}} ; \frac{1}{k_{31}}\right\}
$$$$
\beta_{4} \mid \beta_{(4)}, \delta, \sigma^{2}, y \sim N\left\{\frac{k_{42}}{k_{4 J}} ; \frac{1}{k_{4 I}}\right\}
$$

$$
\beta_{s} \mid \beta_{(5)}, \delta, \sigma^{2}, y \sim N\left\{\frac{k_{52}}{k_{51}} ; \frac{1}{k_{51}}\right\}
$$$$
\beta_{\sigma} \mid \beta_{(\sigma)}, \delta, \sigma^{2}, y \sim N\left\{\frac{k_{62}}{k_{6 I}} ; \frac{l}{k_{\sigma l}}\right\} \text {; }
$$$$
\beta_{7} \mid \beta_{(7)}, \delta, \sigma^{2}, y \sim N\left\{\frac{k_{72}}{k_{71}} ; \frac{l}{k_{71}}\right\} ;
$$ 
$\delta \mid \beta, \sigma^{2}, y \sim N\left\{\frac{r_{12}}{r_{11}} ; \frac{1}{r_{11}}\right\}$

Com os dados, os resultados para $a_{j}=\sum_{i=1}^{n} y_{i} x_{j i}, j=1,2, \ldots, 7 ; b_{j k}=\sum_{i=1}^{n} x_{j i} x_{k i}, j, k=1,2, \ldots, 7 ;$ $c_{j}=\sum_{i=1}^{n} x_{j i} w_{i} ; j=1,2, \ldots, 7 ; f=\sum_{i=1}^{n} y_{i} w_{i} ; e=\sum_{i=1}^{n} w_{l}^{2}$, foram encontradas como sendo:

$a_{1}=0.358 ; \quad a_{2}=0.144 ; \quad a_{3}=0.153 ; \quad a_{4}=0.193 ; \quad a_{5}=0.082 ; \quad a_{6}=0.189 ; \quad a_{7}=0.380 ; \quad b_{11}=1.718 ;$ $b_{12}=b_{21}=0.606 ; \quad b_{13}=b_{31}=0.615 ; \quad b_{14}=b_{41}=1.095 ; \quad b_{15}=b_{51}=0.495 ; \quad b_{16}=b_{61}=0.774 ;$ $b_{17}=b_{71}=1.762 ; \quad b_{22}=0.247 ; \quad b_{23}=b_{32}=0.231 ; \quad b_{24}=b_{42}=0.389 ; \quad b_{25}=b_{52}=0.181$; $b_{26}=b_{62}=0.294 ; \quad b_{27}=b_{72}=0.651 ; \quad b_{33}=0.252 ; \quad b_{34}=b_{43}=0.397 ; \quad b_{35}=b_{53}=0.172 ;$ $b_{36}=b_{63}=0.316 ; \quad b_{37}=b_{73}=0.644 ; \quad b_{44}=0.723 ; \quad b_{45}=b_{54}=0.321 ; \quad b_{46}=b_{64}=0.506 ;$ $b_{47}=b_{74}=1.136 ; \quad b_{55}=0.167 ; \quad b_{56}=b_{65}=0.228 ; \quad b_{57}=b_{75}=0.498 ; \quad b_{66}=0.412 ; \quad b_{67}=b_{76}=0.813 ;$ $b_{77}=1.872 ; \quad c_{1}=1.19 ; \quad c_{2}=0.470 ; \quad c_{3}=0.400 ; \quad c_{4}=0.850 ; \quad c_{5}=0.340 ; \quad c_{6}=0.490 ; \quad c_{7}=1.460 ;$ $f=0.1818 ; e=3$.

Para as observações perdidas na matriz $X$ dada em (6.15) usamos o mesmo procedimento de geração usado para os dados da Tabela 10.

Com as distribuições a priori (6.7) $\operatorname{com} a=0.2, b=0.1, c=2.5$ e $d=2$, geramos cinco cadeias de Gibbs cada uma com 2000 iterações, e após descartar as 400 primeiras observações, tomamos cada décima observação na nossa amostra final. Assim em cinco cadeias, temos uma amostra de 800 observações.

\subsubsection{Resultados}

Na Tabela 14, temos os sumários a posteriori e os valores de $\sqrt{\hat{R}}$ (Critério de Gelman \& Rubin). Para todos os parâmetros, observamos a convergência do algoritmo Gibbs Sampling ( $\sqrt{\hat{\mathrm{R}}}<1.1$ para todos os parâmetros). 
Tabela 14: Sumários a posteriori

\begin{tabular}{cccc}
\hline Parâmetros & Média a posteriori & $\begin{array}{c}\text { Intervalo de } \\
\text { Credibilidade (95\%) }\end{array}$ & $\sqrt{\hat{\boldsymbol{R}}}$ \\
\hline$\beta_{1}$ & 0.0162 & $(-0.1730 ; 0.1986)$ & 1.0000 \\
$\beta_{2}$ & 0.0066 & $(-0.1905 ; 0.2068)$ & 1.0032 \\
$\beta_{3}$ & 0.0127 & $(-0.1667 ; 0.2053)$ & 0.9983 \\
$\beta_{4}$ & 0.0083 & $(-0.2010 ; 0.2062)$ & 1.0017 \\
$\beta_{s}$ & 0.0046 & $(-0.2117 ; 0.1982)$ & 1.0063 \\
$\beta_{6}$ & 0.0067 & $(-0.2052 ; 0.2078)$ & 1.0033 \\
$\beta_{7}$ & 0.0053 & $(-0.1966 ; 0.1872)$ & 1.0000 \\
$\delta$ & 0.0228 & $(-0.2847 ; 0.3240)$ & 1.0038 \\
$\sigma^{2}$ & 0.3689 & $(0.1570 ; 0.8173)$ & 1.0123 \\
\hline
\end{tabular}

Nas Figuras 14 e 15 temos os gráficos das distribuições a posteriori marginais aproximadas pelas amostras geradas pelo algoritmo de Gibbs e gráficos de convergência das amostras geradas.
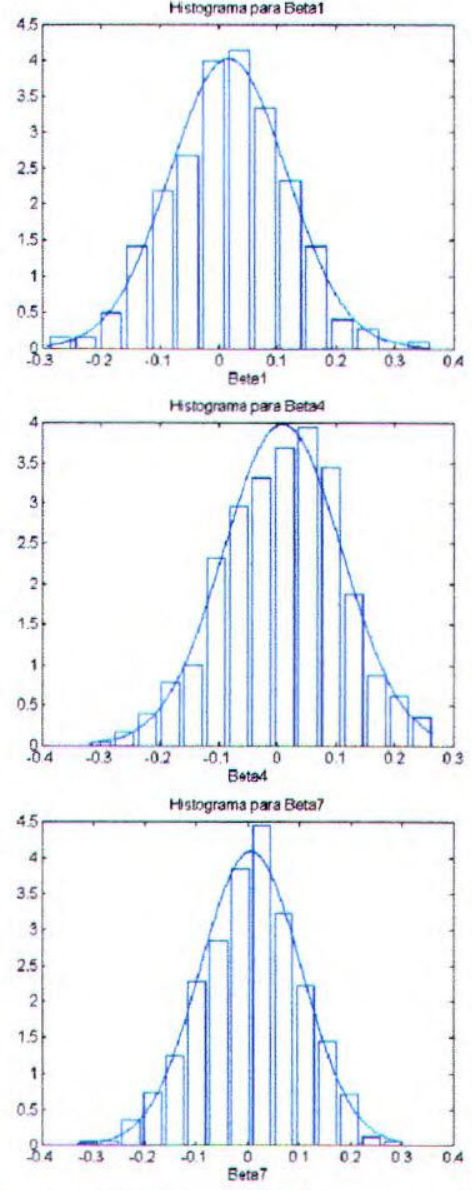

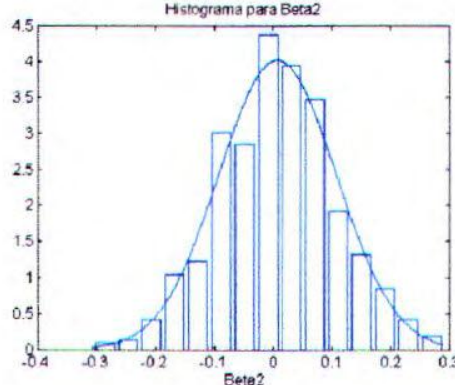

Hstograma para Betas

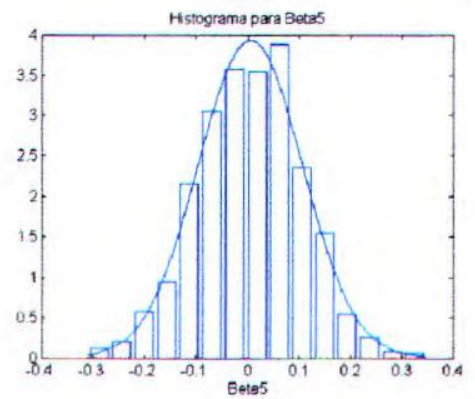

Histograma para det:

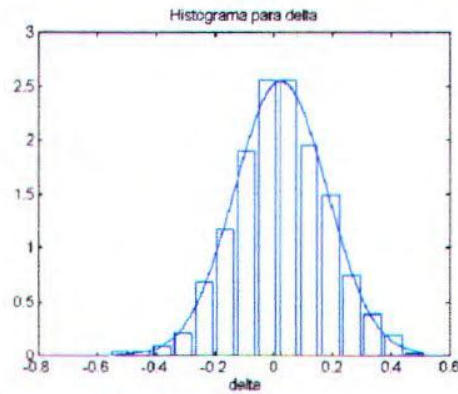

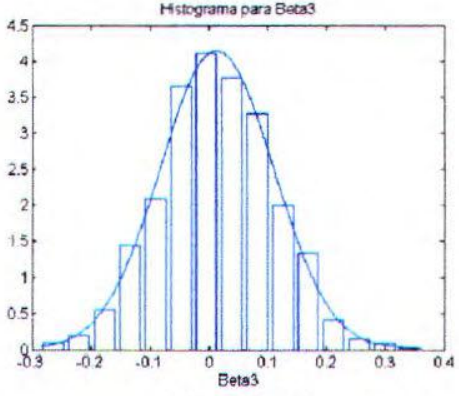

Hstograma para Betra

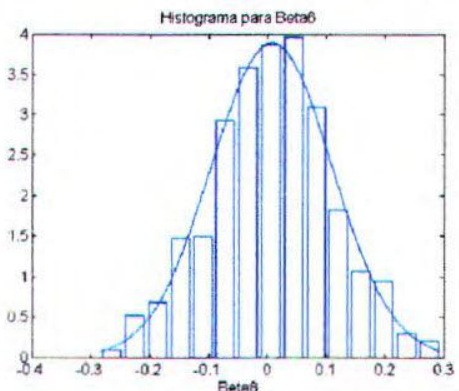

Hostograma para sigme

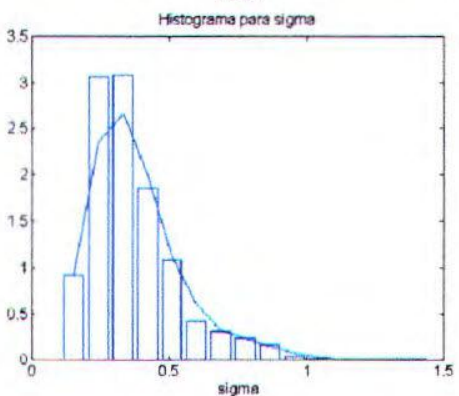

Figura 14: Densidades a posteriori marginais 

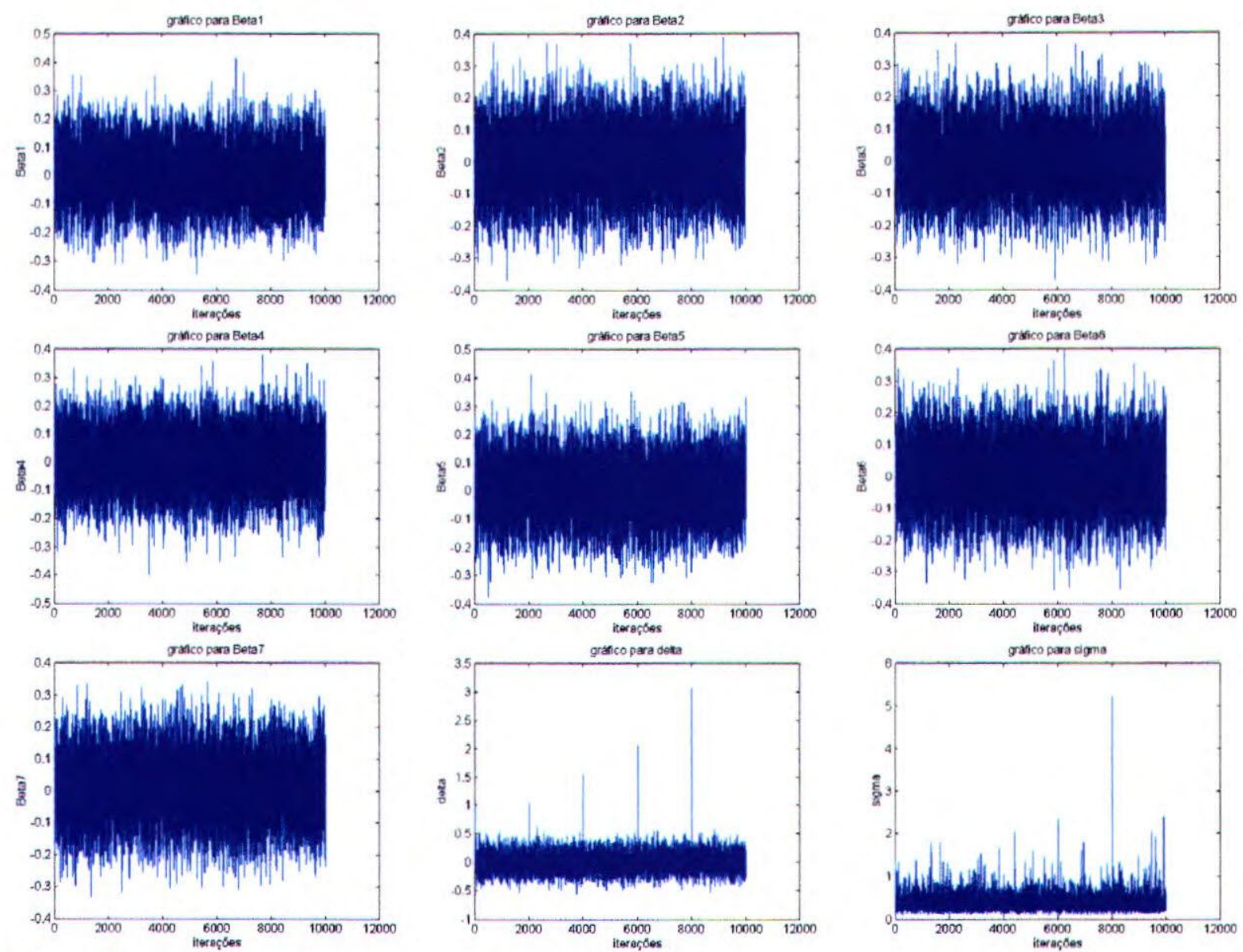

Figura 15: Convergência das amostras geradas

A partir dos resultados dados na Tabela 14, observamos que as covariáveis $x_{1}, x_{2}, \ldots, x_{7}$ não apresentam efeitos significativos na resposta $y=\log (R R)$ onde $R R$ é o risco relativo de câncer entre as usuárias de estrógeno.

Também consideramos o vetor de covariáveis $x_{7}$ denotando a proporção de mulheres que entraram na menopausa com menos de 40 anos por ser um fato menos freqüente e que talvez pudesse apresentar alguma significância, mas não houve nenhuma alteração nas conclusões.

Observando a Tabela 13 percebemos uma possível divisão dos dados com relação a resposta $(\log (R R))$, onde, os laboratórios $1,2,3,4,5$ e 6 apresentam valores baixos para as respostas $(\log (R R))$ quando comparados com os valores referentes aos laboratórios 7, 8 e 9. A partir deste fato, usando o modelo (6.2), tentamos encontrar uma possível diferença entre grupos. 
Consideramos o grupo 1 formado pelos laboratórios $1,2,3,4,5$ e 6 e o grupo 2 formado pelos laboratórios 7,8 e 9 . A partir desta divisão, o vetor $w_{i}=1(0)$, ficou definido como $\underset{\sim}{w^{\prime}}=\left(\begin{array}{lllllll}1 & 1 & 1 & 0 & 0 & 0\end{array}\right)$, onde, $w_{i}=1$ para $i=1,2,3,4,5$ e 6 correspondendo ao grupo $1 \mathrm{e}$ $w_{i}=0$ para $i=7,8$ e 9 correspondendo ao grupo 2 .

Refizemos todas as análises, mas os resultados continuaram de acordo com os resuitados

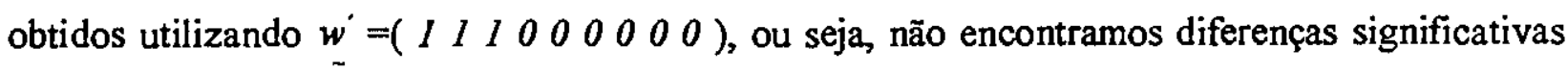
para as covariáveis, nem entre estes supostos grupos.

\subsubsection{Escolha do modelo}

Dentre os modelos utilizados para analisar os dados da Tabela 13, onde, um considera 0 vetor $w^{\prime}=(111000000)($ modelo 1$)$ e o outro considera $w_{\sim}^{\prime}=(111111000)$ (modelo 2), utilizamos o critério proposto na Seção 2.5 para a escolha do melhor modelo.

Através da densidade preditiva ordenada definida na Seção (2.4.1), cujos valores para $c_{\ell}$, $\ell=1,2$ (definido na Seção 2.5) foram encontrados como sendo, $c_{1}=8.414 \times 10^{-38} \mathrm{e}$ $c_{2}=2.4377 \times 10^{-39}$, o modelo escolhido foi o modelo 1 , ou seja, o modelo definido em (6.2) com o vetor $w^{\prime}=(111000000)$

\subsection{Verificação do ajuste do modelo}

Os modelos propostos para analisar os dados das Tabelas 10 e 13 também foram verificados através do procedimento descrito na Seção 2.6.1.

Os valores encontrados para $\hat{p}_{v}$ (definido em (2.11)) usando a estatística de teste definida em (2.10), para cada caso, se encontram dentro do intervalo de aceitação, ou seja, $0.05<\hat{p}_{v}<0.95$ (para cada caso, $\hat{p}_{v}$ se encontrou em tomo de 0.65 ).

Portanto, podemos concluir que o modelo definido em (6.2) está adequado aos dados de forma satisfatória. 


\section{CAPITULO 7 - CONCLUSÕES FINAIS E PROPOSTAS FUTURAS}

O uso de meta-análise tem permitido a combinação de resultados provenientes de vários estudos independentes, possibilitando uma conclusão geral sobre o corpo da pesquisa. A metaanálise tem sido freqüentemente empregada na área médica, principalmente na comparação de grupos de estudo, utilizando, na maioria das vezes a modelagem hierárquica. Tem sido útil também para avaliar se há alguma associação entre uma determinada doença e seus possíveis fatores de risco, como foi exemplificado neste trabalho.

Com o avanço na área computacional, o uso de métodos Bayesianos são facilmente implementados. Em especial, os métodos de simulação de Monte Carlo via Cadeias de Markov (MCMC) têm sido uma alternativa prática de grande interesse nas aplicações de meta-análise. É importante lembrar que na maioria dos casos onde a meta-análise é aplicada, os modelos usados requerem muitos parâmetros, o que torna os métodos clássicos intratáveis.

Além disso, estimadores de Monte Carlo para densidades preditivas são obtidos de maneira simples, permitindo a comparação e discriminação entre os modelos usados.

Como propostas futuras, as técnicas desenvolvidas neste trabalho podem ser estendidas em modelagens de distribuições ponderadas. Este tipo de modelagem pode ser aplicada em muitas situações onde, por exemplo, uma amostra aleatória usual de uma população de interesse não é disponível devido aos dados terem probabilidades desiguais de entrarem na amostra. Existe também a possibilidade de uma amostra aleatória ser possivel, mas o experimentador pode optar 
por não usá-la, desde que uma amostra escolhida cuidadosamente (viciada) pode vir a ser mais informativa (ver Bayarri \& Degroot; 1987).

Outra proposta de estudo é sobre vícios de publicação. Vícios de publicação ocorrem quando pesquisadores ou editores baseiam suas decisões considerando a apresentação ou aceitação de manuscritos. O fato da existência de publicação viciada como um problema sério, já vem sido difundido a muito tempo. Vários exemplos são dados na literatura desde 1661. Neste sentido, uma analogia com distribuições ponderadas pode ser feita para a modelagem de publicações viciadas (ver por exemplo Larose \& Dey (1995 c).

Outros aspectos, tais como, métodos alternativos para testar a convergência dos parâmetros e para a comparação e discriminação dos modelos usados podem ser interessantes. 


\section{APÊNDICE A - O ALGORÍTMO GIBBS SAMPLING}

O Amostrador de Gibbs é um esquema iterativo de amostragem de uma Cadeia de Markov cujo núcleo de transição é formado pelas distribuições condicionais completas.

Para descrever esse algoritmo, supor que $\pi(\theta)$ seja a distribuição de interesse onde $\theta=\left(\theta_{1}, \ldots, \theta_{p}\right)$ e que seja praticamente impossivel gerar amostras diretamente de $\pi(\theta)$; se, as densidades condicionais a posteriori $\pi_{i}\left(\theta_{i}\right)=\pi\left(\theta \mid \theta_{-i}\right), i=1, \ldots, p$ estiverem disponíveis, então podemos gerar amostras de $\pi(\theta)$ através de sucessivas gerações dessas distribuições condicionais $\pi_{\mathrm{i}}\left(\theta_{\mathrm{i}}\right)$.

Este método pode ser descrito da seguinte forma:

Passol: Inicialize 0 contador da cadeia $k=1$ e escolha valores inicias para $\theta^{(0)}=\left(\theta_{1}{ }^{(0)}, \ldots, \theta_{p}{ }^{(0)}\right)$;

Passo 2: Obtenha um novo valor para $\theta^{(\mathbf{k})}=\left(\theta_{1}{ }^{(\mathbf{x})}, \ldots, \theta_{\mathbf{p}}{ }^{(\mathbf{k})}\right)$ a partir de $\theta^{(\mathbf{x - 1 )}}$ através de sucessivas gerações de valores,

$$
\begin{aligned}
& \theta_{1}^{(\mathbf{k})} \sim \pi\left(\theta_{1} \mid \theta_{2}^{(\mathbf{k}-1)}, \ldots, \theta_{\mathrm{p}}^{(\mathrm{k}-1)}\right) \\
& \theta_{2}^{(\mathbf{k})} \sim \pi\left(\theta_{2} \mid \theta_{1}^{(\mathbf{k})}, \ldots, \theta_{\mathrm{p}}^{(\mathrm{k}-1)}\right) \\
& \theta_{3}^{(\mathbf{k})} \sim \pi\left(\theta_{3} \mid \theta_{1}^{(\mathbf{k})}, \theta_{2}^{(\mathbf{k})}, \ldots, \theta_{\mathrm{p}}^{(\mathbf{k}-1)}\right) \\
& \ldots \\
& \theta_{\mathrm{p}}^{(\mathbf{k})} \sim \pi\left(\theta_{\mathrm{p}} \mid \theta_{1}^{(\mathbf{k})}, \theta_{2}^{(\mathbf{k})}, \theta_{3}^{(\mathbf{k})}, \ldots, \theta_{\mathrm{p}-1}^{(\mathbf{k})}\right)
\end{aligned}
$$

Passo3: Atualize o contador de $\mathrm{k}$ para $\mathrm{k}+1$ e retome ao passo2 até atingir a convergência. 


\section{APÊNDICE B - O ALGORITMO METROPOLIS HASTINGS}

Este algoritmo é usado para gerar amostras de uma densidade de forma indireta, quando não se sabe gerar diretamente. Assim, seja a distribuição de interesse denotada por $\pi$ e supor que seja muito difícil ou impossível gerar amostras diretamente de $\pi$, se as densidades condicionais a posteriori não tiverem formas conhecidas, neste caso, usamos o algoritmo MetropolisHastings.

Nesse algoritmo, se no estágio $\mathrm{k}$ a cadeia esta no estado $x$, o próximo estado da cadeia é encontrado gerando um valor candidato $y$ de uma densidade arbitrária $q(x, y)$.Se $q(x, y)$ satisfaz a condição de reversibilidade da cadeia, então:

$q(x, y) \pi(x)=q(y, x) \pi(y)$

e assim temos um núcleo de transição satisfatório e todos os valores gerados serão aceitos. Mas, no entanto, para algum $(x, y)$ podemos ter que

$q(x, y) \pi(x)>q(y, x) \pi(y)$

nesse caso, a cadeia se move de $x$ para $y$ com maior freqüência do que de $y$ para $x$. Par corrigir isso, o número de movimentos de $x$ para $y$ é reduzido introduzindo a probabilidade de ocorrer este movimento $\alpha(x, y)<1$. Se o movimento ocorre, então $x^{(k+1)}=y$, caso contrário $x^{(k+1)}=x^{(k)}$. Assim, temos a relação:

$q(x, y) \pi(x) \alpha(x, y)=q(y, x) \pi(y)$. 
A partir da relação acima, temos que a condição de reversibilidade da cadeia é satisfeita por construção e a probabilidade de aceitação do valor gerado é:

$$
\alpha(x, y)= \begin{cases}\min \left\{\frac{q(y, x) \pi(y)}{q(x, y) \pi(x)}, 1\right\} & \text { se } q(x, y) \pi(x)>0 \\ 1 & \text { caso contrário }\end{cases}
$$

Com as definições acima o algoritmo segue da seguinte forma:

Passol: Inicie o contador da cadeia $\mathrm{k}=1$ e escolha o valor inicial para $x, \dot{x}^{(0)}$;

Passo2: Mova a cadeia para um novo valor $y$ gerado da densidade $q\left(x^{(k-1)}, y\right)$;

Passo 3: Calcule a probabilidade de aceitação do movimento, $\alpha\left(x^{(k-1)}, y\right)$ e comparar com um valor $u$ gerado de uma distribuição Uniforme[0,1]. Se o movimento for aceito, ou seja, $u \leq \alpha\left(x^{(k-1)}, y\right)$, então $x^{(k)}=y$, se não for aceito, $x^{(k)}=x^{(k-1)}$ e a cadeia não se move;

Passo4: Mude o contador de $\mathrm{k}$ para $\mathrm{k}+1$ e retorne ao passo 2 até obter uma distribuição estacionária. 


\section{APÊNDICE C - VERIFICAÇÃO DE CONVERGÊNCIA}

Uma forma de verificar a convergência dos algoritmos Gibbs Sampling e MetropolisHastings é gerar várias cadeias com diferentes condições iniciais para se certificar que se trata de uma Cadeia de Markov irredutível, ou seja, que existe uma distribuição de equilíbrio. Existem vários critérios para verificar a convergência dos algoritmos.

Uma das propostas iniciais de verificação da convergência foi feita informalmente por Gelfand e Smith (1990). Eles sugeriram técnicas gráficas para a verificação da convergência do seguinte modo:

Gerava-se um grande número de iterações $\mathrm{N}$ em $\mathrm{m}$ cadeias paralelas. Com a amostra gerada, construía-se um histograma. O mesmo procedimento é repetido com $\mathrm{N}+\mathrm{k}$ iterações, se não houver diferença perceptivel entre os gráficos, então conclui-se pela convergência das cadeias.

Gelman e Rubin (1992) propuseram um método formal para verificação da convergência.

Esse método é baseado em técnicas de análise de variância e sugere a convergência da cadeia apenas quando a variância entre as cadeias for bem menor que a variância dentro de cada cadeia ou, quando os histogramas das cadeias misturadas são similares ao de cada uma delas isoladamente.

Esse método pode ser descrito da seguinte forma: 
Considerando $\mathrm{m}$ cadeias paralelas em uma função real $t(\theta)$, tem-se $\mathrm{m}$ trajetórias $\left\{f_{i}^{(1)}, t_{i}^{(2)}, \ldots, t_{i}^{(n)}\right\}, \mathrm{i}=1, \ldots, \mathrm{m}$ para cada $t$. Portanto, podem ser obtidas a variância entre as cadeias B e a variância dentro das cadeias $W$, dadas por:

$$
\mathrm{B}=\frac{\mathrm{n}}{\mathrm{m}-1} \sum_{i=1}^{m}\left(\bar{t}_{i}-\bar{t}\right)^{2} \quad \text { e } \quad \mathrm{W}=\frac{1}{\mathrm{~m}(\mathrm{n}-1)} \sum_{i=1}^{m} \sum_{j=1}^{n}\left(t_{i}^{(j)}-\bar{t}_{i}\right)^{2},
$$

onde $\bar{t}_{\imath}$ é a média das observações da cadeia i e $\bar{t}$ é a média dessas médias, $\mathrm{i}=1, \ldots, \mathrm{m}$.

Sob convergência, todos m.n valores são gerados da posteriori e a variância $t$ pode ser estimada de forma não viciada por

$$
\hat{\sigma}^{2}=\frac{\mathrm{n}-1}{\mathrm{n}}(\mathrm{W})+\frac{1}{\mathrm{n}}(\mathrm{B})
$$

e a média desejada pode ser estimada por $\hat{\mu}=\bar{t}$.

Um indicador da convergência é dado pela redução potencial estimada de escala $\mathrm{R}=\sqrt{\hat{\sigma}^{2} / \mathrm{W}}$. Assim a medida que $\mathrm{n}$ cresce, $\mathrm{R}$ convergirá para 1 . Logo, $\mathrm{R}$ pode ser usado como indicador da convergência pela avaliação de sua proximidade a 1 , e então, admitindo que a convergência ocorreu, admitimos também que as amostras das iterações selecionadas são independentes e identicamente distribuídas sobre a distribuição desejada. 


\section{APÊNDICE D - ALGUNS PROGRAMAS DESENVOLVIDOS EM MATLAB}

Neste apêndice, apresentamos alguns programas desenvolvidos no software MATLAB, utilizados para obter amostras da distribuição de interesse através dos algoritmos Gibbs Sampling e Metropolis- Hastings.

1 - Este primeiro programa desenvolvido é referente ao exemplo de aplicação introduzido no capitulo 3 utilizando a reparametrização.

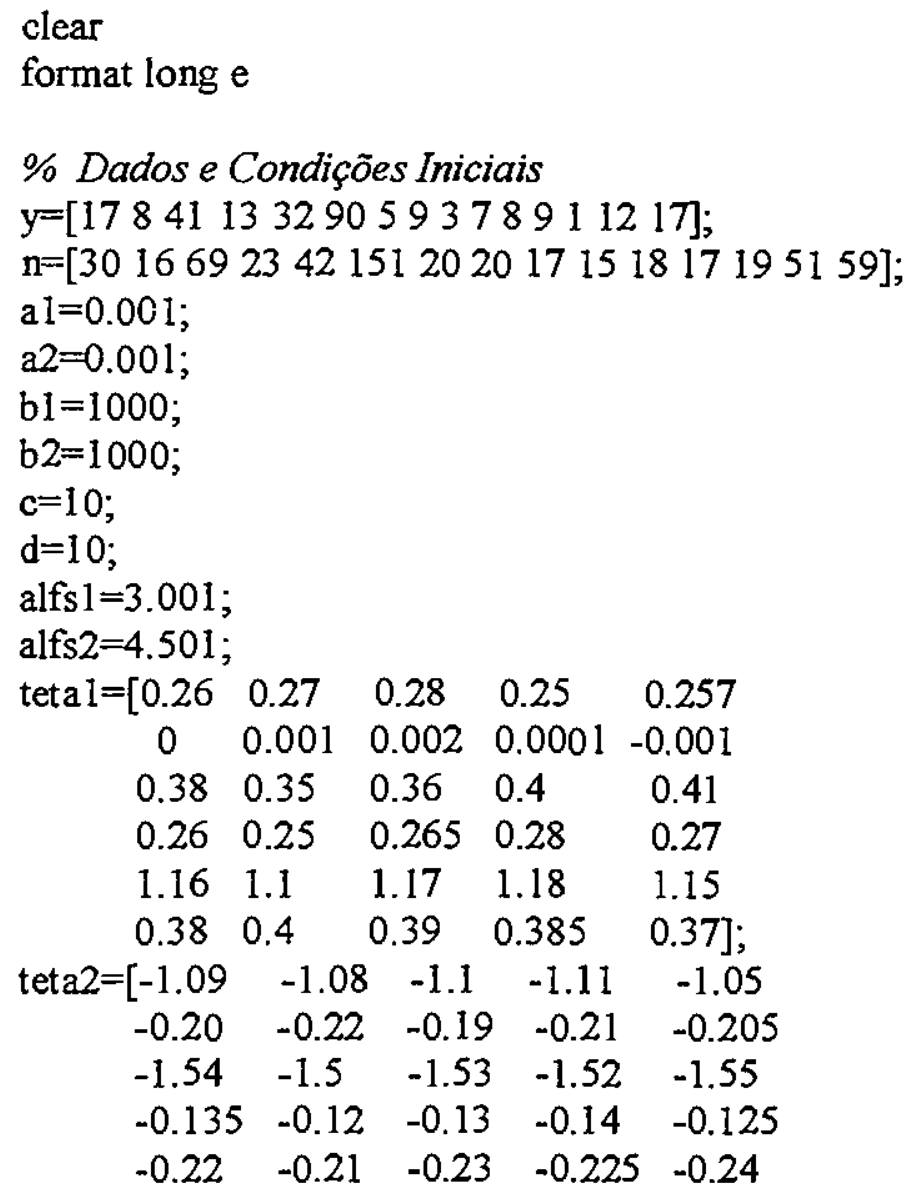




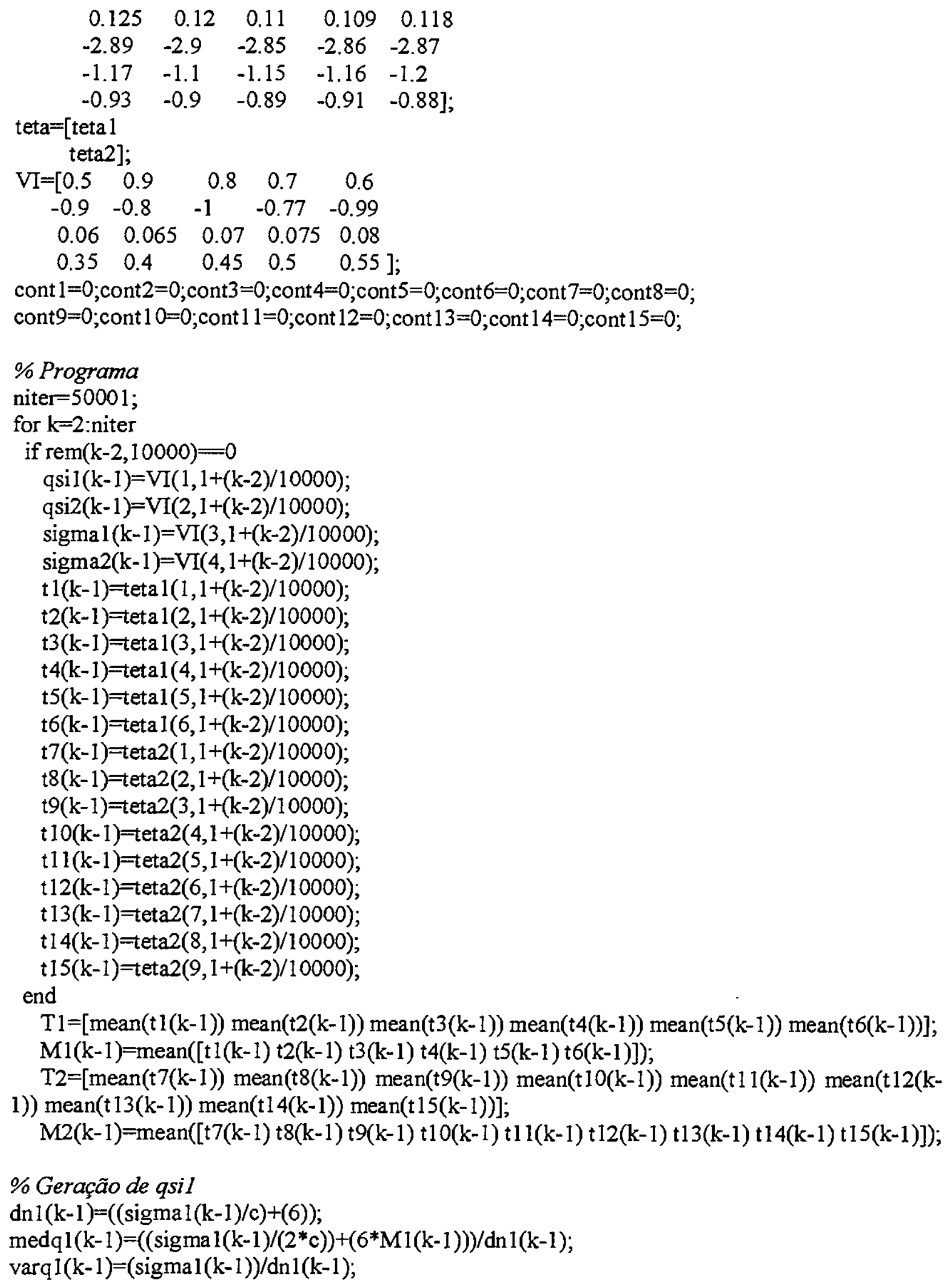




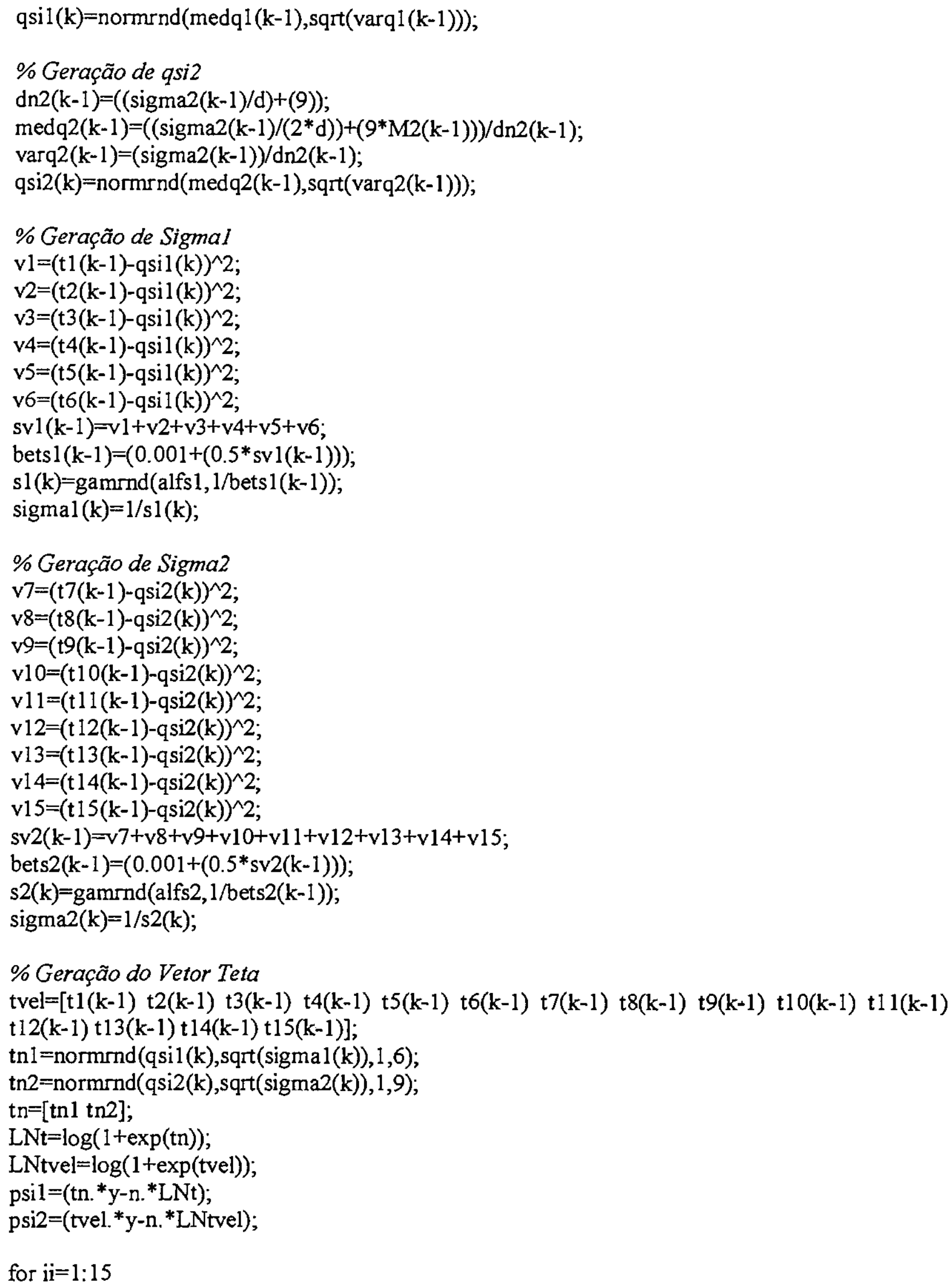

for $\mathrm{ii}=1: 15$ 


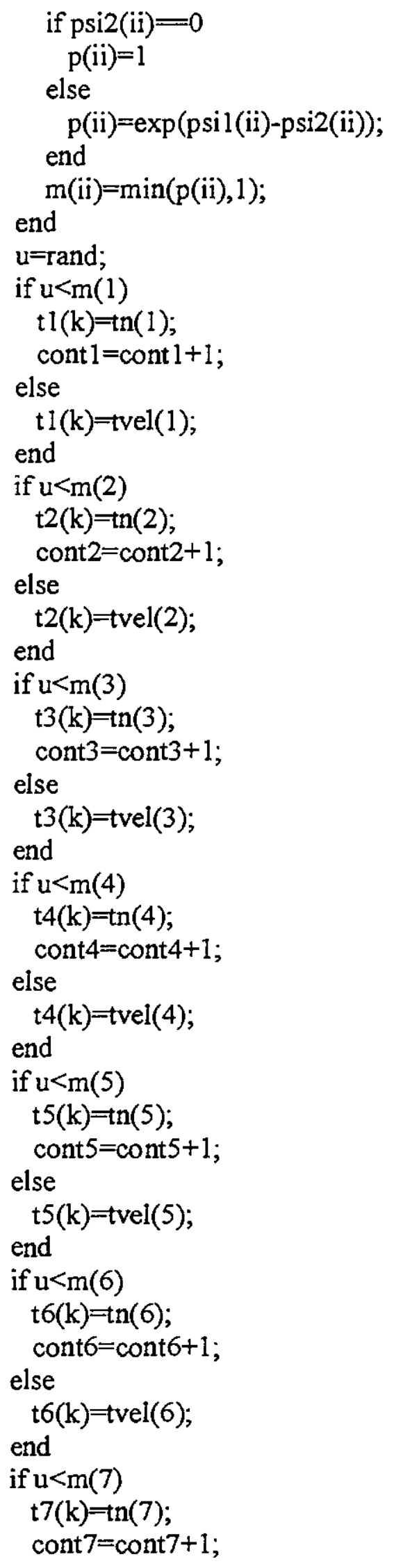




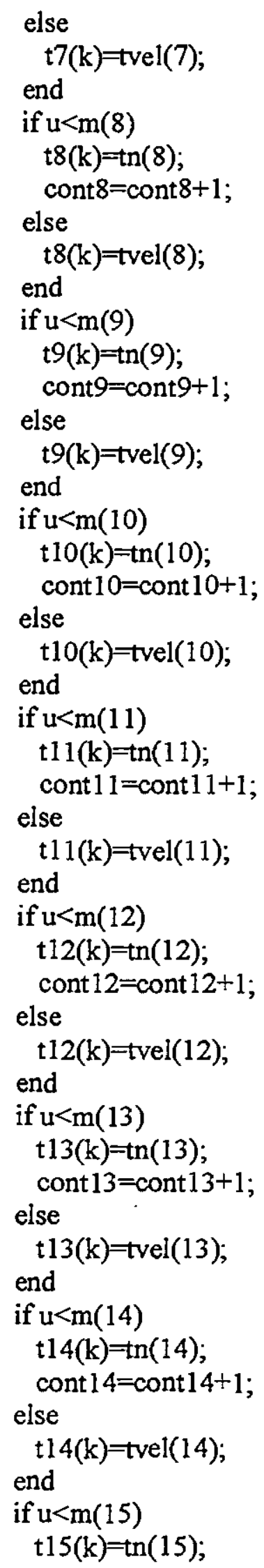




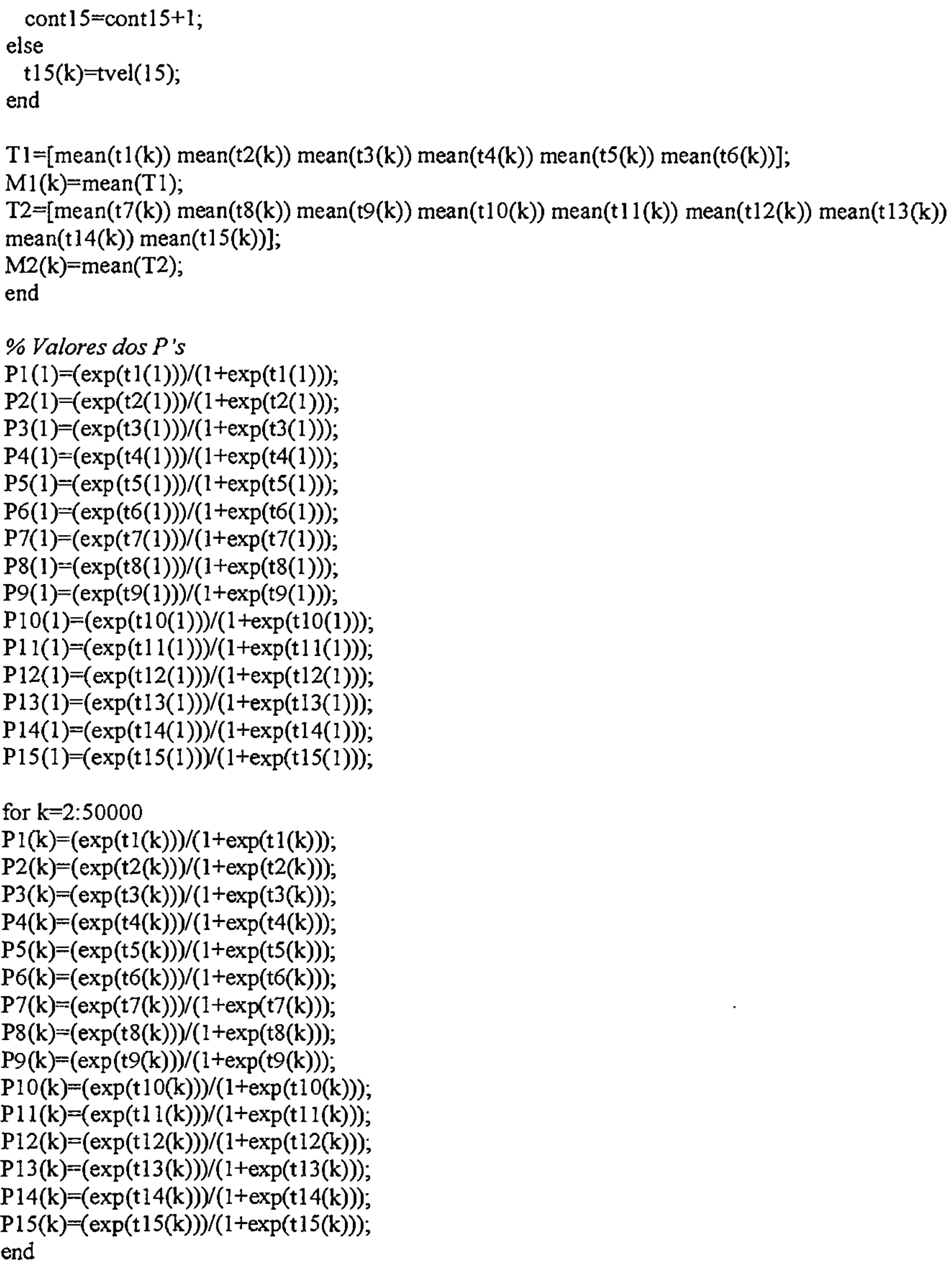


\% Seleção da Amostra e Resultados $\mathrm{g}=1$;

for $j=4001: 10000: 50000$

for $\mathrm{i}=\mathrm{j}: \mathrm{j}+5999$

if $\operatorname{rem}(\mathrm{i}-\mathrm{j}+1,15)=0$

$q \operatorname{sil} s(\mathrm{~g})=\mathrm{qsil}(\mathrm{i})$;

$\mathrm{qsi} 2 \mathrm{~s}(\mathrm{~g})=\mathrm{qsi}(\mathrm{i})$;

$\operatorname{sigmal} \mathrm{s}(\mathrm{g})=\operatorname{sigmal}(\mathrm{i})$;

$\operatorname{sigma} 2 \mathrm{~s}(\mathrm{~g})=\operatorname{sigma} 2(\mathrm{i})$;

$\mathrm{P} 1 \mathrm{~s}(\mathrm{~g})=\mathrm{P} 1(\mathrm{i})$;

$\mathrm{P} 2 \mathrm{~s}(\mathrm{~g})=\mathrm{P} 2(\mathrm{i})$;

$\mathrm{P} 3 \mathrm{~s}(\mathrm{~g})=\mathrm{P} 3(\mathrm{i})$;

$\mathrm{P} 4 \mathrm{~s}(\mathrm{~g})=\mathrm{P} 4(\mathrm{i})$;

$\mathrm{P} 5 \mathrm{~s}(\mathrm{~g})=\mathrm{P} 5(\mathrm{i})$;

P6s $(g)=P 6(i)$;

$\mathrm{P} 7 \mathrm{~s}(\mathrm{~g})=\mathrm{P} 7(\mathrm{i})$;

$\mathrm{P} 8 \mathrm{~s}(\mathrm{~g})=\mathrm{P} 8(\mathrm{i})$;

$\mathrm{P9s}(\mathrm{g})=\mathrm{P9}(\mathrm{i})$;

$\mathrm{P} 10 \mathrm{~s}(\mathrm{~g})=\mathrm{P} 10(\mathrm{i})$;

Pl1s $(\mathrm{g})=\mathrm{P} 11(\mathrm{i})$;

$\mathrm{P} 12 \mathrm{~s}(\mathrm{~g})=\mathrm{P} 12(\mathrm{i})$;

$\mathrm{P} 13 \mathrm{~s}(\mathrm{~g})=\mathrm{P} 13(\mathrm{i})$;

$\mathrm{P} 14 \mathrm{~s}(\mathrm{~g})=\mathrm{P} 14(\mathrm{i})$;

$\mathrm{P} 15 \mathrm{~s}(\mathrm{~g})=\mathrm{P} 15(\mathrm{i})$;

$\mathrm{g}=\mathrm{g}+1$;

end

end

end

\section{\% Inferências}

mqsi $1=[$ mean(qsils) $]$

mqsi2 $=[\operatorname{mean}(q s i 2 s)]$

msigma $1=[$ mean $($ sigmals $)]$

msigma2 $=[$ mean $(\operatorname{sigma} 2 \mathrm{~s})]$

$\mathrm{mP} 1=[\operatorname{mean}(\mathrm{P} 1 \mathrm{~s})]$

$\mathrm{mP} 2=[\operatorname{mean}(\mathrm{P} 2 \mathrm{~s})]$

$\mathrm{mP} 3=[\operatorname{mean}(\mathrm{P} 3 \mathrm{~s})]$

$\mathrm{mP} 4=[\operatorname{mean}(\mathrm{P} 4 \mathrm{~s})]$

$\mathrm{mP5}=[$ mean(P5s)

mP6 $=[$ mean $(\mathrm{P} 6 \mathrm{~s})]$

$\mathrm{mP7}=[$ mean $(\mathrm{P} 7 \mathrm{~s})]$

$\mathrm{mP8}=[$ mean $(\mathrm{P} 8 \mathrm{~s})]$

$\mathrm{mP9}=[$ mean $(\mathrm{P9})]$

$\mathrm{mP} 10=[$ mean $(\mathrm{P} 10 \mathrm{~s})]$

$\mathrm{mP} 11=[\operatorname{mean}(\mathrm{P} 11 \mathrm{~s})]$

$\mathrm{mP} 12=[\operatorname{mean}(\mathrm{P} 12 \mathrm{~s})]$

$\mathrm{mPl} 3=[$ mean $(\mathrm{P} 13 \mathrm{~s})]$ 


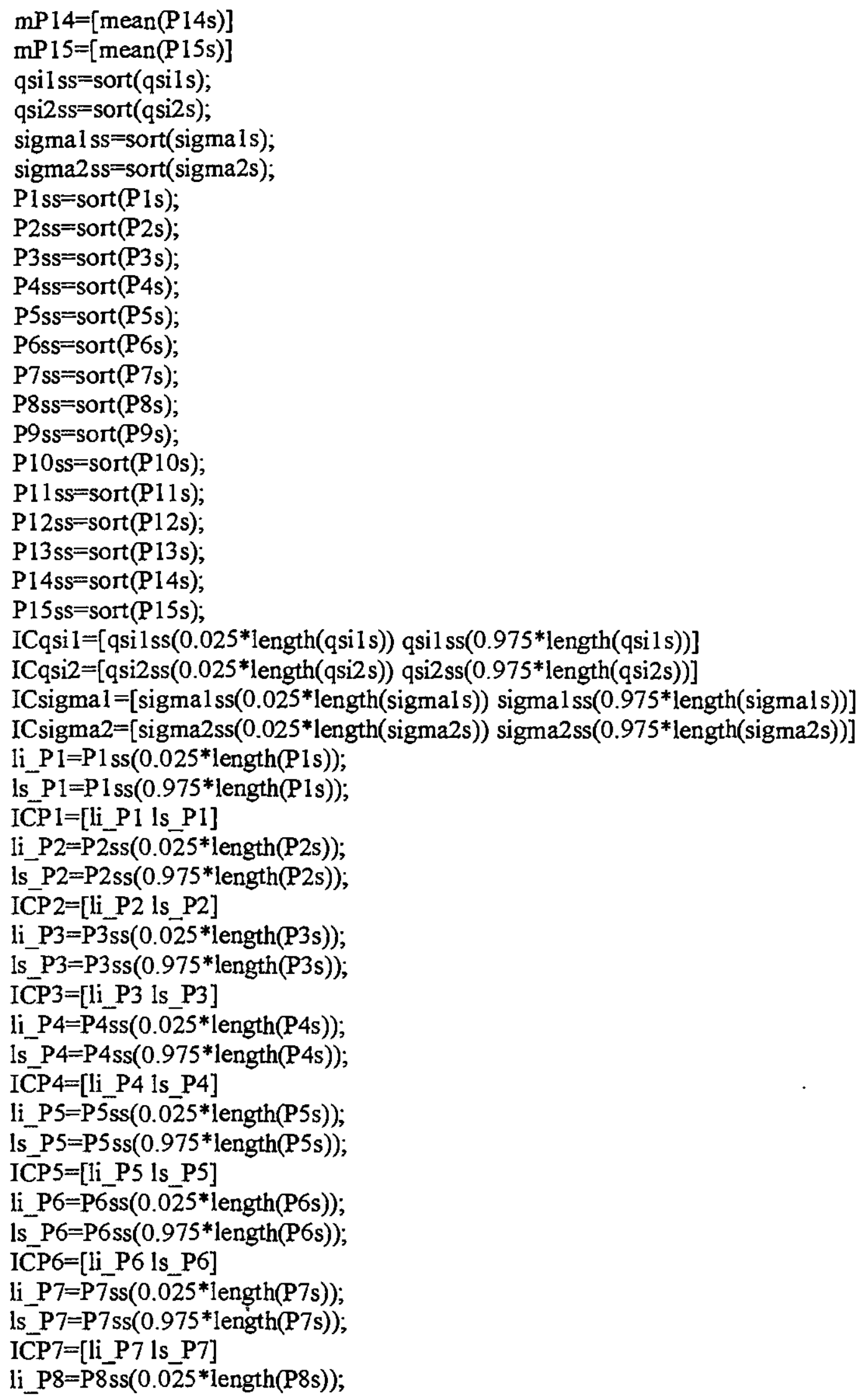




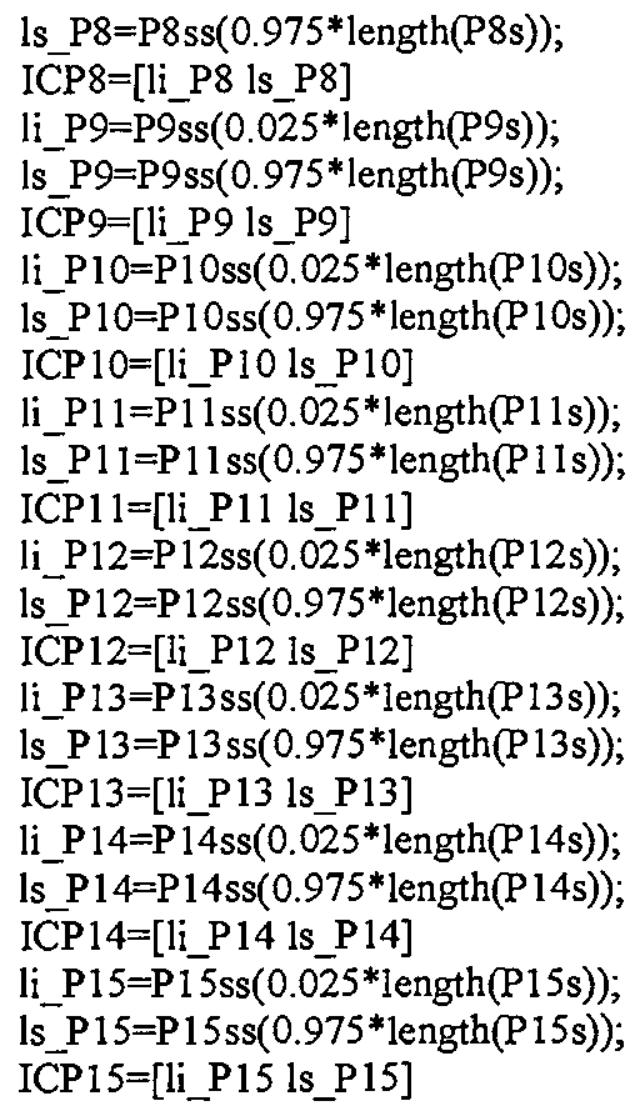




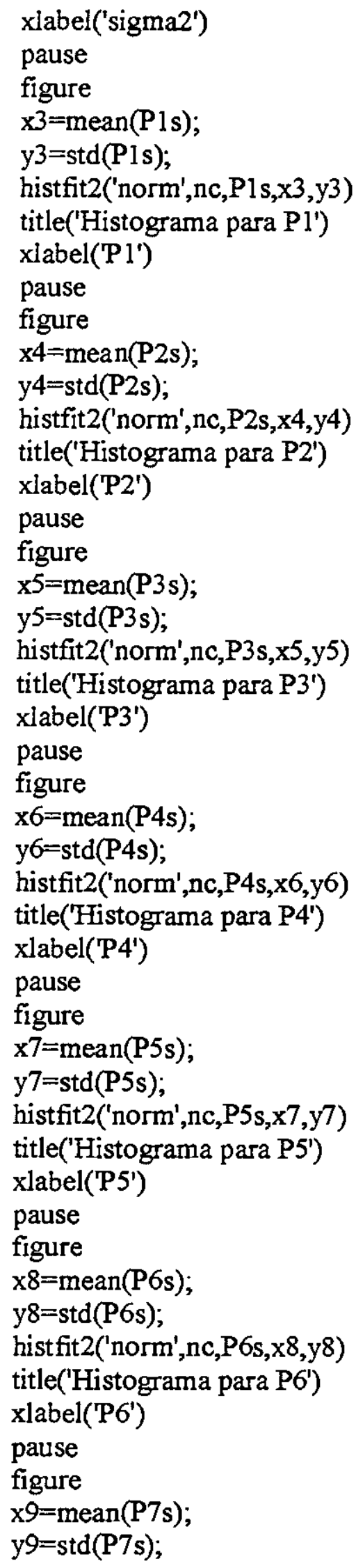




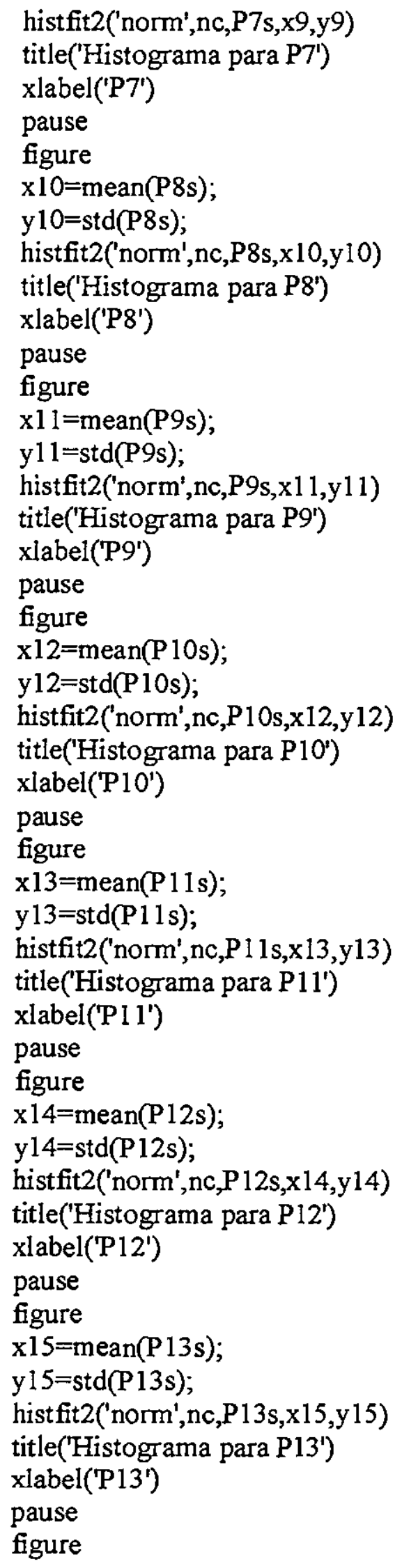




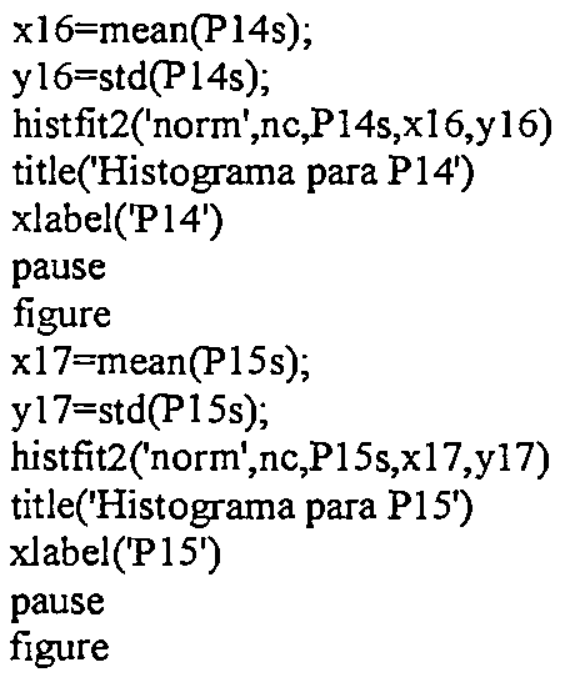

\section{\% Gráficos de Convergência}

plot(qsil)

title('gráfico para qsil')

xlabel('iterações')

ylabel('qsi1')

pause

figure

plot(qsi2)

title('gráfico para qsi2')

xlabel('iterações')

ylabel('qsi2')

pause

figure

plot(sigmal)

title('gráfico para sigmal')

xlabel('iterações')

ylabel('sigma1')

pause

figure

plot(sigma2)

title('gráfico para sigma2')

xlabel('iterações')

ylabel('sigma2')

pause

figure

plot(Pl)

title('gráfico para P1')

xlabel('iterações')

ylabel('P1')

pause

figure

plot(P2) 


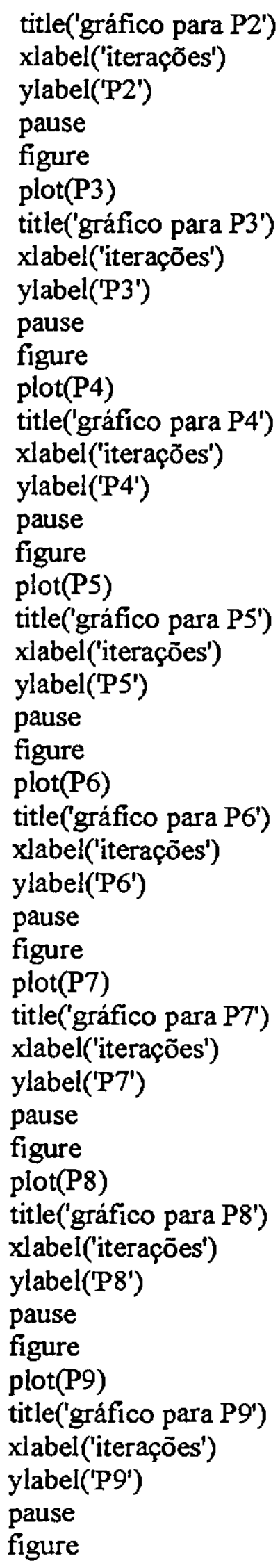




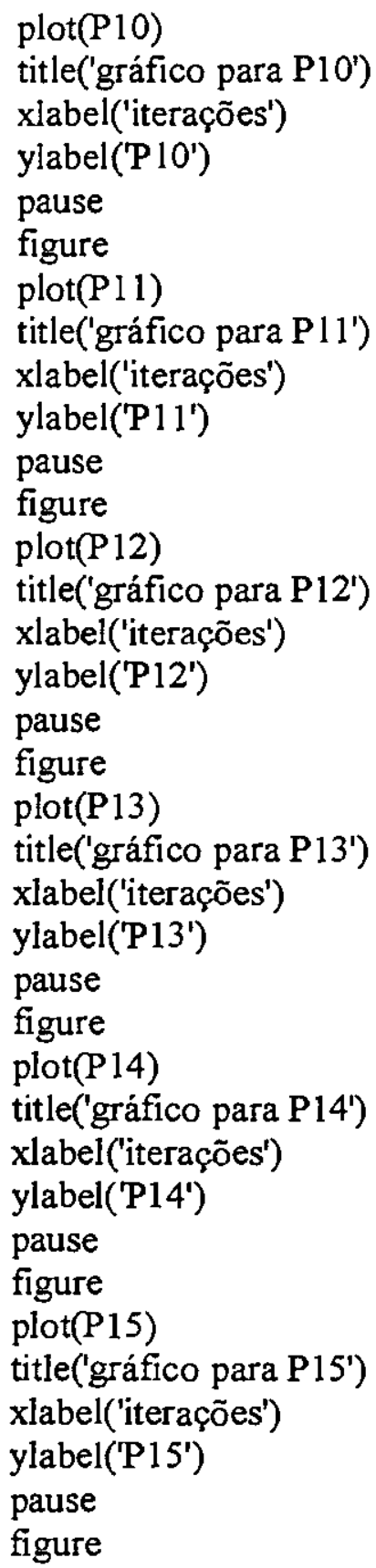


2 - O próximo programa, é referente ao exemplo de aplicação introduzido no capítulo 6 ,

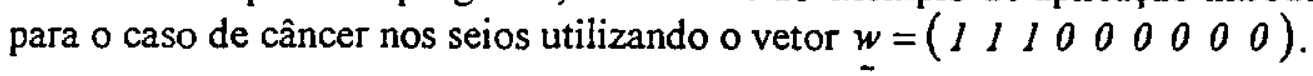

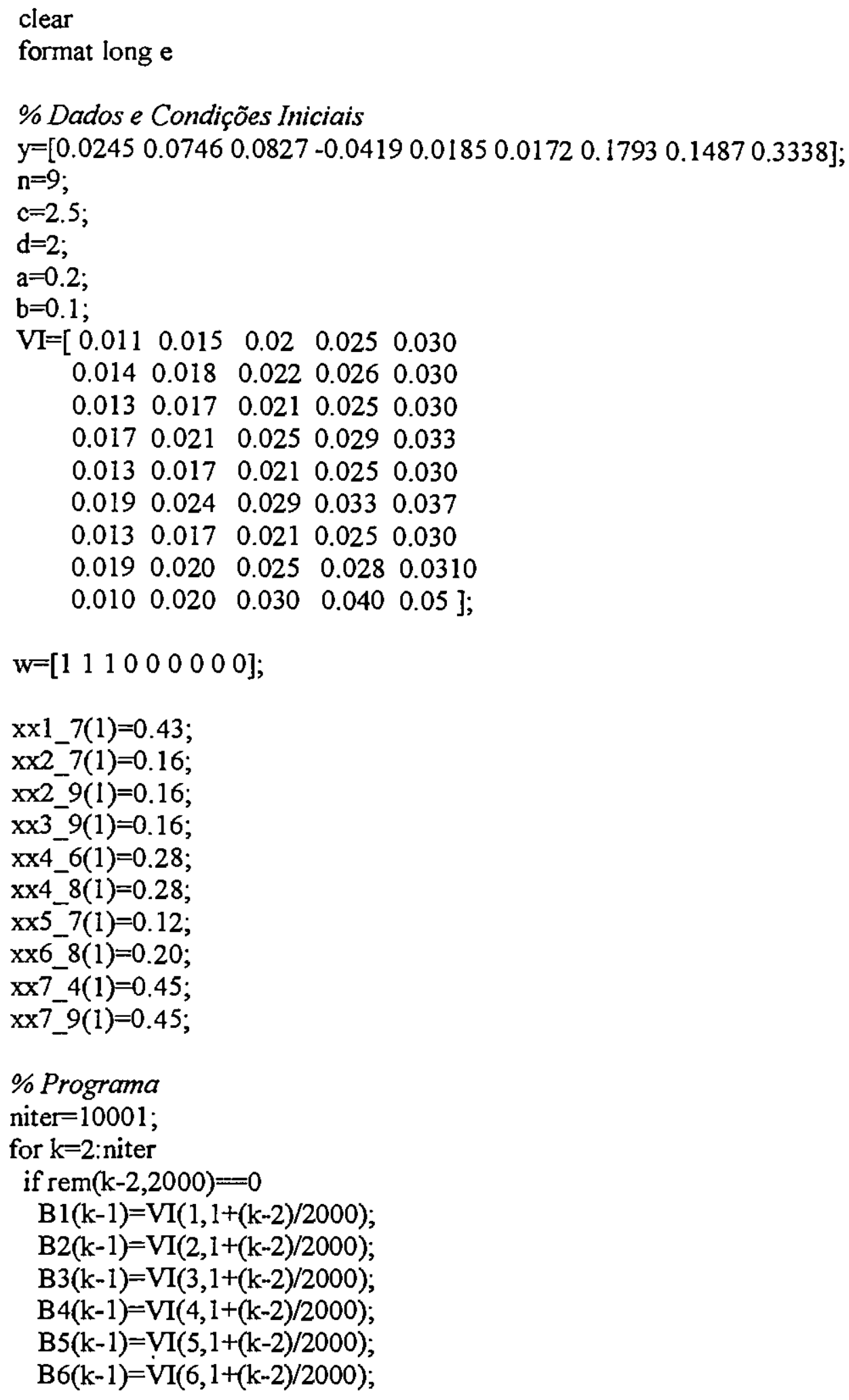




\section{$\mathrm{B} 7(\mathrm{k}-1)=\mathrm{VI}(7,1+(\mathrm{k}-2) / 2000)$; \\ $\operatorname{delta}(\mathrm{k}-1)=\mathrm{VI}(8,1+(\mathrm{k}-2) / 2000)$; \\ $\operatorname{sigma}(\mathrm{k}-1)=\mathrm{VI}(9,1+(\mathrm{k}-2) / 2000)$;}

end

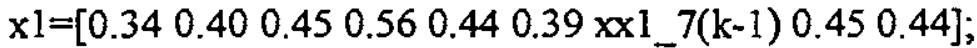

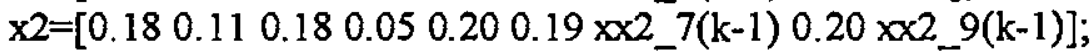

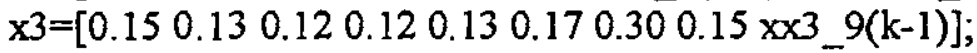

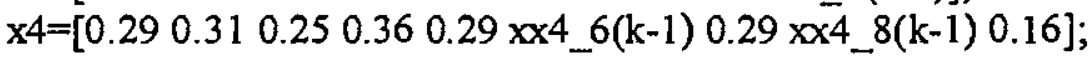

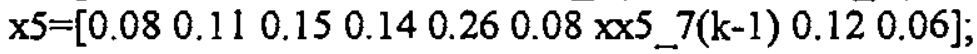

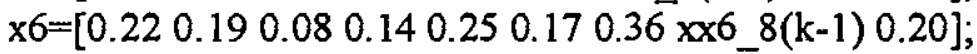

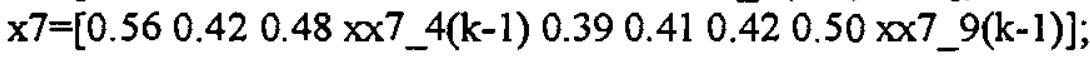

$x \times 1 \_7(\mathrm{k})=$ normrnd $(0.43,0.06)$;

$\mathrm{xx} 2$ 7(k) $=$ normrnd $(0.16,0.06)$;

$\mathrm{xx} 2$ 29(k)=normrnd $(0.16,0.06)$;

$x \times 39(\mathrm{k})=$ normrnd $(0.16,0.06)$;

$\mathrm{xx} 46(\mathrm{k})=$ normrnd $(0.28,0.06)$;

$\mathrm{xx} 4 \_8(\mathrm{k})=$ normrnd $(0.28,0.06)$;

$\mathrm{xx} 5 \_7(\mathrm{k})=$ normrnd $(0.12,0.06)$;

$x \times 6 \_8(\mathrm{k})=$ normrnd $(0.20,0.08)$;

$x \times 7$ _ $4(\mathrm{k})=$ normrnd $(0.45,0.06)$;

$x x 7 \_9(k)=$ normrnd $(0.45,0.06)$;

\section{\% Geração de sigma}

$\mathrm{v} 1=\left(\left(\mathrm{y}(1)-(\mathrm{x} 1(1) * \mathrm{~B} 1(\mathrm{k}-1))-(\mathrm{x} 2(1) * \mathrm{~B} 2(\mathrm{k}-1))-\left(\mathrm{x} 3(1)^{*} \mathrm{~B} 3(\mathrm{k}-1)\right)-(\mathrm{x} 4(1) * \mathrm{~B} 4(\mathrm{k}-1))-(\mathrm{x} 5(1) * \mathrm{~B} 5(\mathrm{k}-1))-\right.\right.$ $(\mathrm{x} 6(1) * \mathrm{~B} 6(\mathrm{k}-1))-(\mathrm{x} 7(1) * \mathrm{~B} 7(\mathrm{k}-1))-(\mathrm{w}(1) * \operatorname{delta}(\mathrm{k}-1))) \wedge 2)$

$\mathrm{v} 2=((\mathrm{y}(2)-(\mathrm{x} 1(2) * \mathrm{~B} 1(\mathrm{k}-1))-(\mathrm{x} 2(2) * \mathrm{~B} 2(\mathrm{k}-1))-(\mathrm{x} 3(2) * \mathrm{~B} 3(\mathrm{k}-1))-(\mathrm{x} 4(2) * \mathrm{~B} 4(\mathrm{k}-1))-(\mathrm{x} 5(2) * \mathrm{~B} 5(\mathrm{k}-1))-$ $\left.(\mathrm{x} 6(2) * \mathrm{~B} 6(\mathrm{k}-1))-(\mathrm{x} 7(2) * \mathrm{~B} 7(\mathrm{k}-1))-(\mathrm{w}(2) * \operatorname{delta}(\mathrm{k}-1)))^{\wedge} 2\right)$;

$\mathrm{v} 3=((\mathrm{y}(3)-(\mathrm{xl}(3) * \mathrm{~B} 1(\mathrm{k}-1))-(\mathrm{x} 2(3) * \mathrm{~B} 2(\mathrm{k}-1))-(\mathrm{x} 3(3) * \mathrm{~B} 3(\mathrm{k}-1))-(\mathrm{x} 4(3) * \mathrm{~B} 4(\mathrm{k}-1))-(\mathrm{x} 5(3) * \mathrm{~B} 5(\mathrm{k}-1))-$ $(\mathrm{x} 6(3) * \mathrm{~B} 6(\mathrm{k}-1))-(\mathrm{x} 7(3) * \mathrm{~B} 7(\mathrm{k}-1))-(\mathrm{w}(3) * \operatorname{delta}(\mathrm{k}-1))) \wedge 2)$;

$\mathrm{v} 4=\left(\left(\mathrm{y}(4)-(\mathrm{x} 1(4) * \mathrm{~B} 1(\mathrm{k}-1))-(\mathrm{x} 2(4) * \mathrm{~B} 2(\mathrm{k}-1))-(\mathrm{x} 3(4) * \mathrm{~B} 3(\mathrm{k}-1))-\left(\mathrm{x} 4(4)^{*} \mathrm{~B} 4(\mathrm{k}-1)\right)-(\mathrm{x} 5(4) * \mathrm{~B} 5(\mathrm{k}-1))-\right.\right.$ $\left.(\mathrm{x} 6(4) * \mathrm{~B} 6(\mathrm{k}-1))-(\mathrm{x} 7(4) * \mathrm{~B} 7(\mathrm{k}-1))-(\mathrm{w}(4) * \operatorname{delta}(\mathrm{k}-1)))^{\wedge} 2\right)$;

$\mathrm{v} 5=((\mathrm{y}(5)-(\mathrm{x} 1(5) * \mathrm{~B} 1(\mathrm{k}-1))-(\mathrm{x} 2(5) * \mathrm{~B} 2(\mathrm{k}-1))-(\mathrm{x} 3(5) * \mathrm{~B} 3(\mathrm{k}-1))-(\mathrm{x} 4(5) * \mathrm{~B} 4(\mathrm{k}-1))-(\mathrm{x} 5(5) * \mathrm{~B} 5(\mathrm{k}-1))-$ $\left.(\mathrm{x} 6(5) * \mathrm{~B} 6(\mathrm{k}-1))-(\mathrm{x} 7(5) * \mathrm{~B} 7(\mathrm{k}-1))-(\mathrm{w}(5) * \operatorname{delta}(\mathrm{k}-1)))^{\wedge} 2\right)$;

v6 $=((\mathrm{y}(6)-(\mathrm{xl}(6) * \mathrm{~B} 1(\mathrm{k}-1))-(\mathrm{x} 2(6) * \mathrm{~B} 2(\mathrm{k}-1))-(\mathrm{x} 3(6) * \mathrm{~B} 3(\mathrm{k}-1))-(\mathrm{x} 4(6) * \mathrm{~B} 4(\mathrm{k}-1))-(\mathrm{x} 5(6) * \mathrm{~B} 5(\mathrm{k}-1))-$ $\left.(\mathrm{x} 6(6) * \mathrm{~B} 6(\mathrm{k}-1))-(\mathrm{x} 7(6) * \mathrm{~B} 7(\mathrm{k}-1))-(\mathrm{w}(6) * \operatorname{delta}(\mathrm{k}-1)))^{\wedge} 2\right)$;

$\mathrm{v} 7=\left(\left(\mathrm{y}(7)-\left(\mathrm{xl}(7)^{*} \mathrm{~B} 1(\mathrm{k}-1)\right)-(\mathrm{x} 2(7) * \mathrm{~B} 2(\mathrm{k}-1))-\left(\mathrm{x} 3(7)^{*} \mathrm{~B} 3(\mathrm{k}-1)\right)-\left(\mathrm{x} 4(7)^{*} \mathrm{~B} 4(\mathrm{k}-1)\right)-(\mathrm{x} 5(7) * \mathrm{~B} 5(\mathrm{k}-1))-\right.\right.$ $\left.(\mathrm{x} 6(7) * \mathrm{~B} 6(\mathrm{k}-1))-(\mathrm{x} 7(7) * \mathrm{~B} 7(\mathrm{k}-1))-(\mathrm{w}(7) * \operatorname{delta}(\mathrm{k}-1)))^{\wedge} 2\right)$;

$\mathrm{v} 8=\left(\left(\mathrm{y}(8)-(\mathrm{x} 1(8) * \mathrm{~B} 1(\mathrm{k}-1))-(\mathrm{x} 2(8) * \mathrm{~B} 2(\mathrm{k}-1))-\left(\mathrm{x} 3(8)^{*} \mathrm{~B} 3(\mathrm{k}-1)\right)-(\mathrm{x} 4(8) * \mathrm{~B} 4(\mathrm{k}-1))-(\mathrm{x} 5(8) * \mathrm{~B} 5(\mathrm{k}-1))-\right.\right.$ $\left.\left.(\mathrm{x} 6(8) * \mathrm{~B} 6(\mathrm{k}-1))-\left(\mathrm{x} 7(8)^{*} \mathrm{~B} 7(\mathrm{k}-1)\right)-(\mathrm{w}(8) * \operatorname{delta}(\mathrm{k}-1))\right)^{\wedge} 2\right)$;

v9= $\left(\left(\mathrm{y}(9)-(\mathrm{x} 1(9) * \mathrm{~B} 1(\mathrm{k}-1))-(\mathrm{x} 2(9) * \mathrm{~B} 2(\mathrm{k}-1))-\left(\mathrm{x} 3(9)^{*} \mathrm{~B} 3(\mathrm{k}-1)\right)-\left(\mathrm{x} 4(9)^{*} \mathrm{~B} 4(\mathrm{k}-1)\right)-(\mathrm{x} 5(9) * \mathrm{~B} 5(\mathrm{k}-1))-\right.\right.$ $(\mathrm{x} 6(9) * \mathrm{~B} 6(\mathrm{k}-1))-(\mathrm{x} 7(9) * \mathrm{~B} 7(\mathrm{k}-1))-(\mathrm{w}(9) * \operatorname{delta}(\mathrm{k}-1))) \wedge 2)$;

soma $(k-1)=v 1+v 2+v 3+v 4+v 5+v 6+v 7+v 8+v 9$;

asigma $=((\mathrm{n} / 2)+\mathrm{c})$;

bsigma $(k-1)=(d+(0.5 * \operatorname{soma}(k-1)))$;

$\operatorname{sig}(\mathrm{k})=$ gamrnd(asigma, 1/(bsigma(k-1)));

$\operatorname{sigma}(k)=(1 / \operatorname{sig}(k))$; 


\section{$\%$ Geração de Betal}

$\mathrm{k} 1 \mathrm{l}=\left(1 /\left((\mathrm{b})^{\wedge} 2\right)\right)+(1.718 / \operatorname{sigma}(\mathrm{k}))$;

$\mathrm{k} 12=(((0.358)) / \operatorname{sigma}(\mathrm{k}))-(((0.606) * \mathrm{~B} 2(\mathrm{k}-1)) / \operatorname{sigma}(\mathrm{k}))-(((0.615) * \mathrm{~B} 3(\mathrm{k}-1)) / \operatorname{sigma}(\mathrm{k}))-$

$(((1.095) * \mathrm{~B} 4(\mathrm{k}-1)) /$ sigma(k))-(((0.495)*B5(k-1))/sigma(k))-(((0.774)*B6(k-1))/sigma(k))$(((1.762) * \mathrm{~B} 7(\mathrm{k}-1)) / \operatorname{sigma}(\mathrm{k}))-(((1.190) * \operatorname{delta}(\mathrm{k}-1)) /$ sigma $(\mathrm{k}))$;

$\mathrm{Bl}(\mathrm{k})=$ normrnd $((\mathrm{k} 12 /(\mathrm{k} 11))$, sqrt $(1 / \mathrm{k} 11))$;

$\%$ Geração de Beta2

$\mathrm{k} 21=\left(1 /\left((\mathrm{b})^{\wedge} 2\right)\right)+(0.247 / \mathrm{sigma}(\mathrm{k}))$;

$\mathrm{k} 22=(((0.144)) / \operatorname{sigma}(\mathrm{k}))-(((0.606) * \mathrm{~B} 1(\mathrm{k}-1)) / \operatorname{sigma}(\mathrm{k}))-(((0.231) * \mathrm{~B} 3(\mathrm{k}-1)) / \mathrm{sigma}(\mathrm{k}))-$

$\left(\left((0.389)^{*} \mathrm{~B} 4(\mathrm{k}-1)\right) / \mathrm{sigma}(\mathrm{k})\right)-\left(\left((0.181)^{*} \mathrm{~B} 5(\mathrm{k}-1)\right) / \operatorname{sigma}(\mathrm{k})\right)-(((0.294) * \mathrm{~B} 6(\mathrm{k}-1)) / \mathrm{sigma}(\mathrm{k}))$ $(((0.651) * \mathrm{~B} 7(\mathrm{k}-1)) / \operatorname{sigma}(\mathrm{k}))-(((0.470) * \operatorname{delta}(\mathrm{k}-1)) / \mathrm{sigma}(\mathrm{k}))$;

$\mathrm{B} 2(\mathrm{k})=$ normrnd $((\mathrm{k} 22 /(\mathrm{k} 21))$, sqrt $(1 / \mathrm{k} 21))$;

\section{$\%$ Geração de Beta3}

$\mathrm{k} 3 \mathrm{l}=\left(1 /\left((\mathrm{b})^{\wedge} 2\right)\right)+(0.252 /$ sigma $(\mathrm{k}))$;

$\mathrm{k} 32=(((0.153)) / \operatorname{sigma}(\mathrm{k}))-\left(\left((0.615)^{*} \mathrm{~B} 1(\mathrm{k}-1)\right) / \operatorname{sigma}(\mathrm{k})\right)-(((0.231) * \mathrm{~B} 2(\mathrm{k}-1)) / \mathrm{sigma}(\mathrm{k}))-$

$(((0.397) * \mathrm{~B} 4(\mathrm{k}-1)) /$ sigma(k))-(((0.172)*B5(k-1))/sigma(k))-(((0.316)*B6(k-1))/sigma(k))$(((0.644) * \mathrm{~B} 7(\mathrm{k}-1)) / \operatorname{sigma}(\mathrm{k}))-\left(\left((0.400)^{*} \operatorname{delta}(\mathrm{k}-1)\right) / \operatorname{sigma}(\mathrm{k})\right)$;

$\mathrm{B} 3(\mathrm{k})=$ normrnd $((\mathrm{k} 32 /(\mathrm{k} 31))$,sqrt $(1 / \mathrm{k} 31))$;

\section{$\%$ Geração de Beta4}

$\mathrm{k} 41=\left(1 /\left((\mathrm{b})^{\wedge} 2\right)\right)+(0.723 /$ sigma $(\mathrm{k}))$;

$\mathrm{k} 42=(((0.193)) / \mathrm{sigma}(\mathrm{k}))-\left(\left((1.095)^{*} \mathrm{~B} 1(\mathrm{k}-1)\right) / \operatorname{sigma}(\mathrm{k})\right)-(((0.389) * \mathrm{~B} 2(\mathrm{k}-1)) / \mathrm{sigma}(\mathrm{k}))-$

$(((0.397) * \mathrm{~B} 3(\mathrm{k}-1)) /$ sigma(k) $)-(((0.321) * \mathrm{~B} 5(\mathrm{k}-1)) /$ sigma(k) $)-(((0.506) * \mathrm{~B} 6(\mathrm{k}-1)) /$ sigma $(\mathrm{k}))-$ $(((1.136) * \mathrm{~B} 7(\mathrm{k}-1)) / \operatorname{sigma}(\mathrm{k}))-\left(\left((0.850)^{*} \operatorname{delta}(\mathrm{k}-1)\right) / \operatorname{sigma}(\mathrm{k})\right)$;

$\mathrm{B} 4(\mathrm{k})=$ normmd $((\mathrm{k} 42 /(\mathrm{k} 41))$,sqrt $(1 / \mathrm{k} 41))$;

$\%$ Geração de Beta5

$\mathrm{k} 5 \mathrm{l}=\left(\mathrm{l} /\left((\mathrm{b})^{\wedge} 2\right)\right)+(0.167 /$ sigma $(\mathrm{k}))$;

$\mathrm{k} 52=(((0.082)) / \operatorname{sigma}(\mathrm{k}))-(((0.495) * \mathrm{~B} 1(\mathrm{k}-1)) / \operatorname{sigma}(\mathrm{k}))-\left(\left((0.181)^{*} \mathrm{~B} 2(\mathrm{k}-1)\right) / \operatorname{sigma}(\mathrm{k})\right)-$

$(((0.172) * \mathrm{~B} 3(\mathrm{k}-1)) /$ sigma $(\mathrm{k}))-(((0.321) * \mathrm{~B} 4(\mathrm{k}-1)) / \mathrm{sigma}(\mathrm{k}))-(((0.228) * \mathrm{~B} 6(\mathrm{k}-1)) / \mathrm{sigma}(\mathrm{k}))-$ $(((0.498) * \mathrm{~B} 7(\mathrm{k}-1)) /$ sigma(k))-(((0.340)*delta(k-1))/sigma(k));

$\mathrm{B} 5(\mathrm{k})=$ normrnd $((\mathrm{k} 52 /(\mathrm{k} 51))$,sqrt $(1 / \mathrm{k} 51))$;

\section{\% Geração de Beta6}

$\mathrm{k} 61=\left(1 /\left((\mathrm{b})^{\wedge} 2\right)\right)+(0.412 /$ sigma $(\mathrm{k}))$;

$\mathrm{k} 62=(((0.189)) / \operatorname{sigma}(\mathrm{k}))-(((0.774) * \mathrm{~B} 1(\mathrm{k}-1)) / \mathrm{sigma}(\mathrm{k}))-(((0.294) * \mathrm{~B} 2(\mathrm{k}-1)) / \mathrm{sigma}(\mathrm{k}))-$

$\left(\left((0.316)^{*} \mathrm{~B} 3(\mathrm{k}-1)\right) /\right.$ sigma(k)) $-(((0.506) * \mathrm{~B} 4(\mathrm{k}-1)) /$ sigma(k) $)-(((0.228) * \mathrm{~B} 5(\mathrm{k}-1)) /$ sigma $(\mathrm{k}))-$ $(((0.813) * \mathrm{~B} 7(\mathrm{k}-1)) /$ sigma(k) $)-(((0.490) * \operatorname{delta}(\mathrm{k}-1)) /$ sigma $(\mathrm{k}))$;

$B 6(k)=$ normrnd $((k 62 /(k 61))$,sqrt $(1 / k 61))$;

\section{$\%$ Geração de Beta7}

$\mathrm{k} 71=\left(1 /\left((\mathrm{b})^{\wedge} 2\right)\right)+(1.872 /$ sigma $(\mathrm{k}))$;

$\mathrm{k} 72=(((0.380)) / \operatorname{sigma}(\mathrm{k}))-(((1.762) * \mathrm{~B} 1(\mathrm{k}-1)) / \operatorname{sigma}(\mathrm{k}))-(((0.651) * \mathrm{~B} 2(\mathrm{k}-1)) /$ sigma $(\mathrm{k}))-$

$(((0.644) * \mathrm{~B} 3(\mathrm{k}-1)) /$ sigma(k) $)-\left(\left((1.136)^{*} \mathrm{~B} 4(\mathrm{k}-1)\right) /\right.$ sigma(k) $)-\left(\left((0.498)^{*} \mathrm{~B} 5(\mathrm{k}-1)\right) /\right.$ sigma(k) $)-$ $(((0.813) * \mathrm{~B} 6(\mathrm{k}-1)) / \operatorname{sigma}(\mathrm{k}))-(((1.460) * \operatorname{delta}(\mathrm{k}-1)) / \operatorname{sigma}(\mathrm{k})) ;$ 


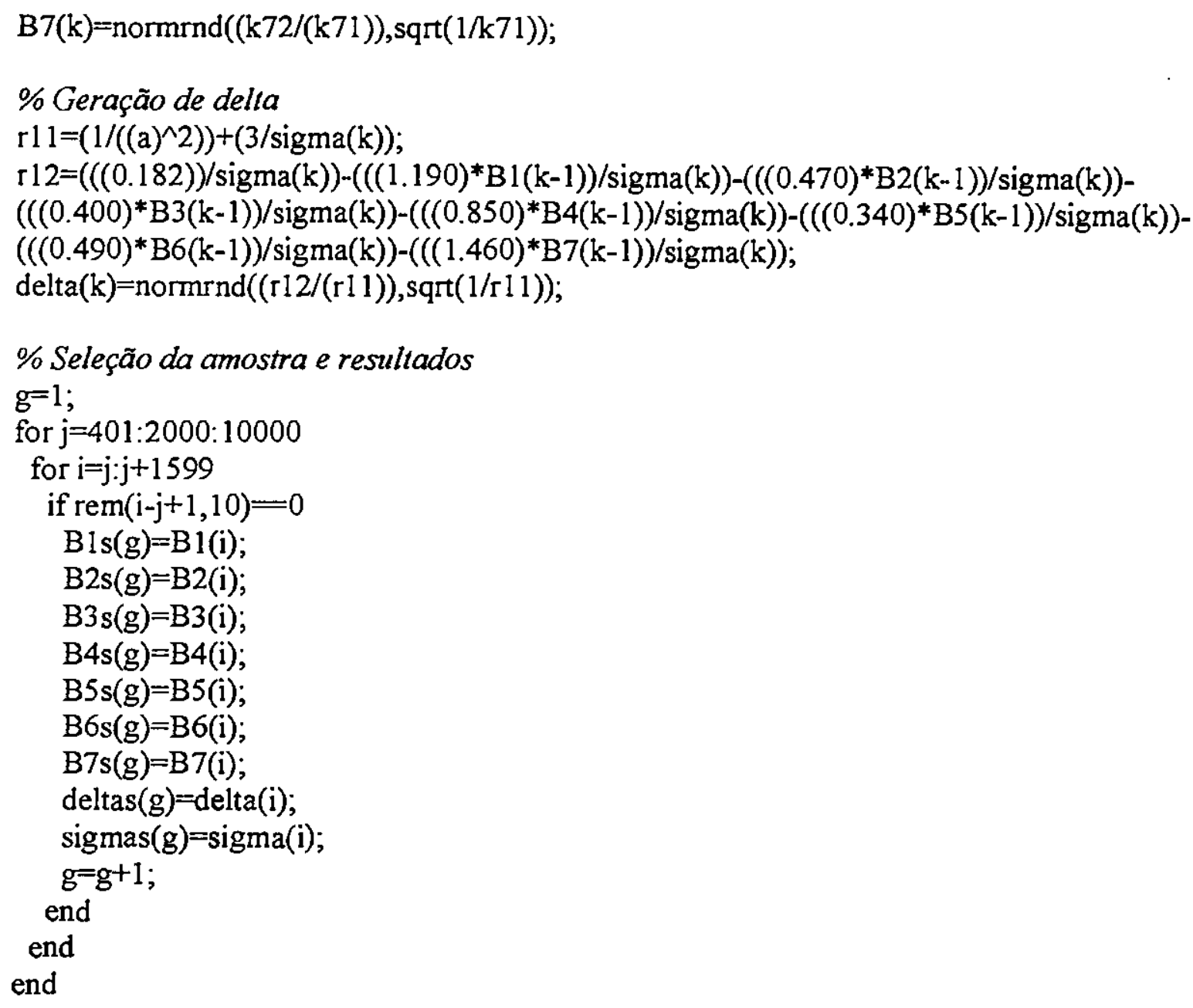



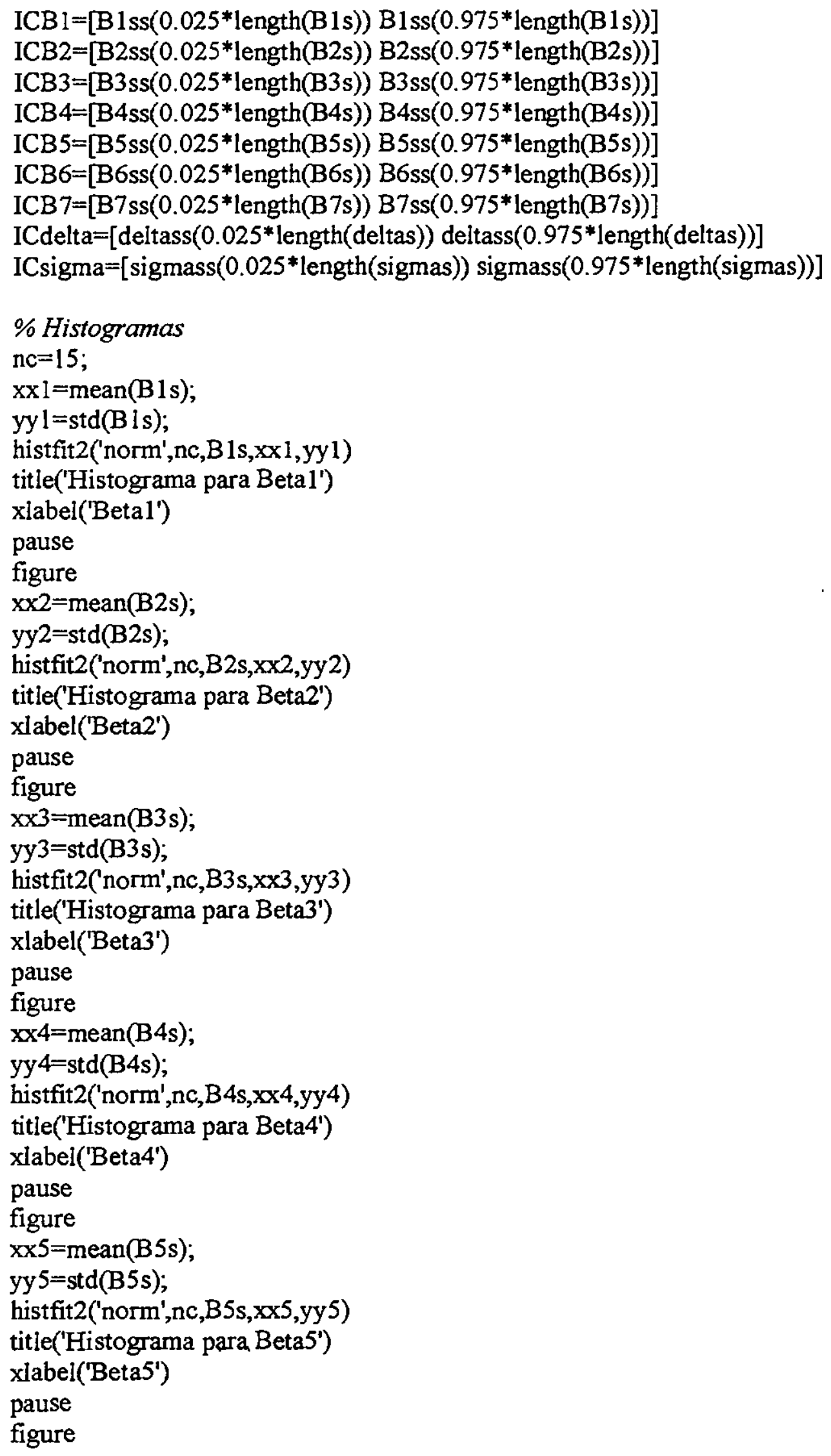


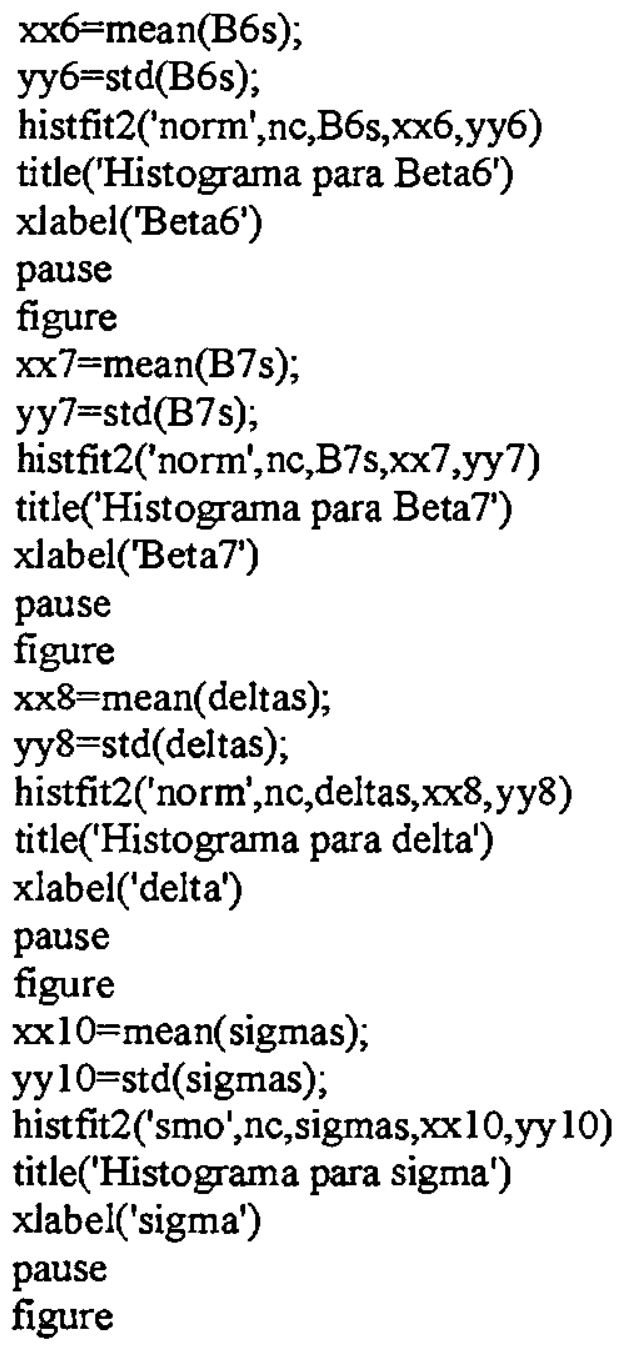

$\%$ Gráficos de convergência

plot(B1)

title('gráfico para Betal')

xlabel('iterações')

ylabel('Betal')

pause

figure

plot(B2)

title('gráfico para Beta2')

xlabel('iterações')

ylabel('Beta2')

pause

figure

plot(B3)

title('gráfico para Beta3')

xlabel('iterações')

ylabel('Beta3')

pause 


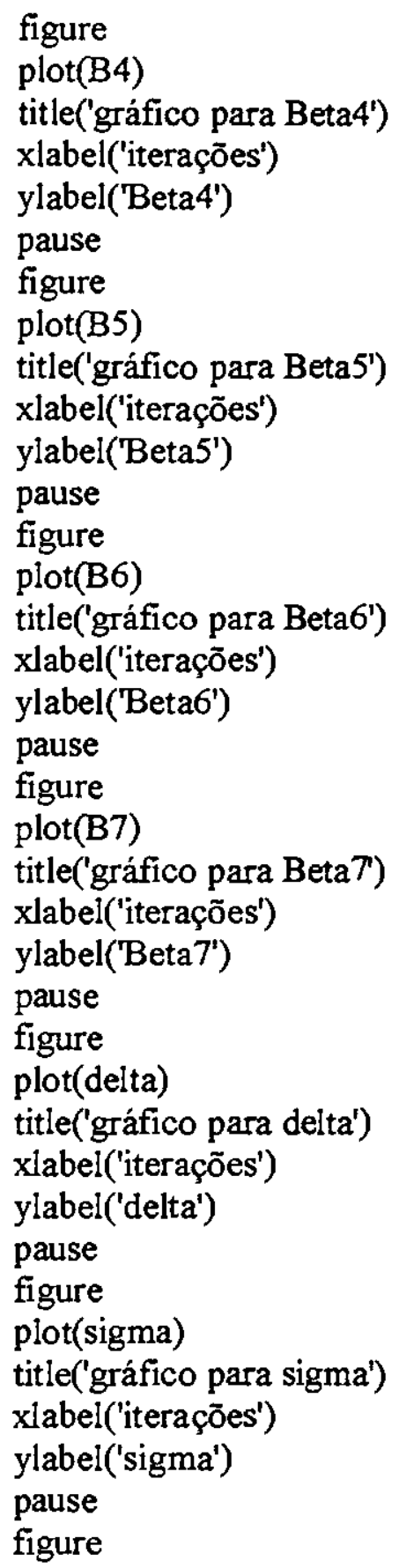




\section{REFERÊNCIAS BIBLIOGRÁFICAS}

ADAMI, H. O. (1992). Long-term consequences of estrogen and estrogen-progestin replacement. Cancer Causes and Control, 3, 83-90.

AITKIN, M. (1991). Posterior factor. J. R. Stat. Soc., B, 53, 111-142.

BAYARRI, M. J. ; DeGROOT, M. H. (1987). Information in selection models, probability and bayesian statistics (R. Viertl, ed.), New York:Plenum Press, 39-51.

BERGER, J. O.; PERICCHI, L. R. (1993). The intrinsic Bayes factor for model selection and prediction. Technical Report, Dep. of Statistcs, Purdue University.

BIGGERSTAFF, B. J.; TWEEDIE, R. L. (1997). Incorporating variability in estimates of heterogeneity in the random effects model in meta-Analysis. Statistics in Medicine, 16, 753-768.

BOX, G. E. P.; HILL, W. J. (1967). Discrimination among mechanism models. Technometrics, $9,57-71$.

BRINTON, L. A.; SCHAIRER, C. (1993). Estrogen replacement therapy and breast cancer risk. Epidemiologic Reviews, 15, 66-79.

BULPITT, C. J. (1988). Meta-analysis. Lancet, ii, 93-94.

CARLIN, B. P.; LOUIS, T. A. (1997). Bayes and empirical Bayes Methods for data analysis. London, Chapman \& Hall.

CASELlA, G.; GEORGE, E. I. (1992). Explaining the Gibbs Sampler. The American Statistician, 46, 167-174.

CHIB, S.; GREENBERG, E. (1995). Understand the Metropolis-Hastings algorithm. The American Statistician, 49, 4, 327-335. 
COCHRAN, W. G. (1937). Problems arising in the analysis of a series of similar experiments. J. Royal Stat. Soc. B.

DuMOUCHEL, W. H. (1994). Predictive cross-valudation in Bayesian meta-analysis. Fifth International Conference on Bayesian Statistical". Alicante, Spain.

DuMOUCHEL, W. H.; HARRIS, J. E. (1983). Bayes methods for combining the results of cancer studies in humans and other species. Journal of the American Statistical Association, 78, 293-315

FISHER, L. D. ; VAN BELLE, G. (1993). Biostatistics: A methodology for health sciences. New York: John Wiley and Sons.

FISHER, R. A. (1932). Statistical methods for research workers (4th ed.). London:Oliver \& Boyd.

FLEISS, J. L. (1993). The statistical basis of meta-Analysis. Statistical Methods in Medical Research, 2, 147-160.

FLEISS, J. L.(1973). Statistical methods for rates \& proportions. John Wiley \& Sons.

GAMERMAN, D. (1996). Simulação estocástica via Cadeias de Markov. 12 SINAPESimpósio Nacional de Probabilidade e Estatística, Caxambú, Brasil.

GEISSER, S.; EDDY, W. F. (1979). A predictive approach to model Selection. J. Am. Stat. Assoc., 74, 153-160.

GELFAND, " E.; DEY, D. K. (1994). Bayesian model choice: Asymptotic and exact calculations. J. R. Stat. Soc., B, 3, 501-514.

GELFAND, A. E.; SMTTH, A. F. M. (1990). Sampling-based approaches to calculating marginal densities. Journal of the American Statistical Association, 85, 398-409.

GELMAN, A.; CARLIN, J. B.; STERN, H. S.; RUBIN, D. B. (1995). Bayesian data analysis. London, Chapman \& Hall.

GELMAN, A. E.; RUBIN, D. (1992). Inference from iterative simulation using multiple sequences. Statistical Science, 7, 457-472.

GEORGE, E. O. ; MUDHOLKAR, G. S. (1977). The logit method for combining independent tests. Institute of Mathematical Statistics Bulletin, 6, 212.

GLASS, G. V. (1976). Primary, secondary, and meta-analysis of research. Educational Researcher, 5, 3-8.

GLASS, G. V.; McGRAW, B.; SMITH, M. L. (1981). Meta-analysis in social research. Beverly Hills, Calif., Sage Publications. 
HEDGES , L. U. (1992). Meta-analysis. Joumal of Educational Statistics, 17, 229-296.

HEDGES, L. V. ; OLKIN, I. (1985). Statistical methods for meta-analysis. Orlando, F. L.: Academic Press.

HEDGES , L. U. (1983). Combining independent estimators in research synthesis. Br. J. Math. Stat. Psychol, 36, 123-131.

HEDGES , L. U. (1982). Estimation of effect size from a series of independent experiments. Psychol Buil, 92, 490-499.

HUNTER, J. E.; SCHIMIDT, F. L.; JACKSON, B. G. (1982). Meta-analysis: Cumulating research findings across studies. Beverly Hills, Calif. Sage Publications.

JEFFREYS, H. (1939). Theory of probability. Oxford University Press.

KAUFMAN, D. W. et al (1991). Estrgen replacement therapy and the risk of breast cancer: results from the case control surveillance study. American Joumal of Epidemiology, 134, 1375-1385.

KELSEY, J. L.; WHITTEMORE, A. S. (1994). Epidemiology and primary prevention of cancers of the breast, endometrium, and ovary. Annals of Epidemiology, 4, 89-95.

JCK, S. S., WALKER, A. M.; JCK, H. (1993). Estrogens, progesterone and endometrial cancer. Epidemiology, 4, 20-24.

L'ABBE, K. A.; DETSKY, A. S., O'ROURKE, K. (1987). Meta-analysis in clinical research. Ann. Intern. Med., 107, 224-233.

LAROSE, D. T.; DEY, D. K. (1997). Grouped randon effects models for Bayesian metaanalysis. Statistics in Medicine, 16, 1817-1829.

LAROSE, D. T. (1996). Bayesian approaches to meta-analysis. PhD Thesis, Departament of Statistcs, University of Connecticut, Storrs, U.S.A.

LAROSE, D. T.; DEY, D. K. (1995 a). Grouped randon effects models for Bayesian metaanalysis. Technical Report, Department of Statistics, University of Connecticut.

LAROSE, D. T.; DEY, D. K. (1995 b). Is estrogen linked to cancers of the breast and endometrium? New meta-analyses using Bayesian grouped random effects model. Relatório técnico 95-35, departamento de estatística, Universidade de Connecticut, Storrs.

LAROSE, D. T.; DEY, D. K. (1995 c). Modeling publication bias using weighted distributions in a Bayesian framework. Rel. técnico, Universidade de Connecticut, Departamento de Estatística. 
LIGHT, R. J.; PILLEMER, D. B. (1984). Summing up: The Science of reviewing research. Cambridge, Mass, Harvard University Press.

MOOD, A. M. ; GRAYBILL, F. A.; BOES, D. C. (1974). Introduction to the theory of statistics. (32. ed.), New York, McGraw-Hill.

OLKIN, I. (1992). Meta-analysis: methods for combining independent stidies. Statistical Science, 7, 226.

PEARSON, E. S. (1938). The probability integral transformation for testing goodness of fit and combining independent tests of significance. Biometrika, 30, 134-148.

PETITTI, D. B. (1994). Meta-analysis, decision analysis and cost effectiveness analysis. Oxford, Oxford University Press.

PRESS, S. J. (1989). Bayesian statistcs:principles, models and applications. John Wiley \& Sons.

ROBERTS, G. O.; SMITH, A. F. M. (1993). Bayesian methods via the Gibbs Sampling and related Markov Chain Monte Carlo methods. Journal of the Royal Statistical Society, B, 55, 3-23.

ROSENTHAL, R. (1978). Combining results of independent studies. Psychol Bull, 85, 185-193.

SACKS, H. S. et al (1988). Meta-analysis of randomized controlled trials. N. Engl. J. Med., 316, $450-455$.

SILLIMAN, N. P.; (1997). Hierarchical selection models with applications in meta-analysis. Journal of the American Statistical Association, 92, 926-936.

SMITH, D. et al (1975). Association of exogenous estrogen and endometrial carcinoma. New England Journal of Medicine, 293, 1164.

STOUFFER, S. A. ; SUCHMAN, E. A. ; DeVINNEY, L. C. ; STAR, S. A. ; WILLIAMS, R. M. Jr. (1949). The american soldier, Vol. 1. Adjustment durin Army Life. Princeton: Princeton University Press.

TIERNEY, L.; KADANE, J. B. (1986). Accurate aproximations for posteriori moments and marginal densities. Journal of the American Statistical Associations, 81, 82-86.

TIPPETT, L. H. C. (1931). The method of statistics. London: Williams \& Norgate.

ZIEL, H. K; FINKLE, W. D. (1975). Association of estrone with the development of endometrial carcinoma. American Journal of Obstetrics and Gynecology, 124, 735-740. 